\title{
PARABOLIC INDUCTIONS FOR PRO-p-IWAHORI HECKE ALGEBRAS
}

\author{
NORIYUKI ABE
}

\begin{abstract}
We give some properties of parabolic inductions and their adjoint functors for pro- $p$-Iwahori Hecke algebras.
\end{abstract}

\section{INTRODUCTION}

Let $F$ be a non-archimedean local field with residue characteristic $p, G$ a connected reductive group over $F$ and $C$ a commutative ring. Fix a pro- $p$ Iwahori subgroup $I(1)$ of $G$ and let $\mathcal{H}$ be the space of $I(1)$-biinvariant $C$ valued functions with compact support. Then via the convolution product, $\mathcal{H}$ has the structure of an algebra, called pro-p-Iwahori Hecke algebra.

We are mainly interested in "mod $p$ case", namely when $C$ is an algebraically closed field of characteristic $p$. In this case, this algebra has an important role to study modulo $p$ representations of $G$. For example, in the proof of the classification theorem [AHHV], it has a crucial role. Motivated by this, we study the representation theory of $\mathcal{H}$.

As usual in the theory of reductive groups, the parabolic induction is one of the most important tool in the study of $\mathcal{H}$-modules. This functor is studied in Oll10, Abe, Vig15b. In particular, using parabolic induction, the simple $\mathcal{H}$-modules are classified in terms of supersingular modules Abe, Theorem 1.1]. (Note that the supersingular modules are classified by Ollivier Oll14 and Vignéras Vig15a.) This is an analogous of the main theorem of $\mathrm{AHHV}$.

In this paper, we study more details of the parabolic induction.

1.1. Results. Let $P$ be a parabolic subgroup and $\mathcal{H}_{P}$ the pro-p-Iwahori Hceke algebra of its Levi part. Then the parabolic induction $I_{P}$ is defined by

$$
I_{P}(\sigma)=\operatorname{Hom}_{\left(\mathcal{H}_{P}^{-}, j_{P}^{-*}\right)}(\mathcal{H}, \sigma)
$$

using "negative subalgebra" $\mathcal{H}_{P}^{-}$of $\mathcal{H}_{P}$ and a certain algebra homomorphism $j_{P}^{-*}: \mathcal{H}_{P}^{-} \rightarrow \mathcal{H}$. Replacing "negative subalgebra" with "positive subalgebra" $\mathcal{H}_{P}^{+}$, we also have another notion of "inductions". Moreover, we also have other maps $j_{P}^{ \pm}: \mathcal{H}_{P}^{ \pm} \rightarrow \mathcal{H}$. Therefore, we have four "inductions". We compare these inductions and give relations. It turns out that we only have two inductions $I_{P}$ and $I_{P}^{\prime}=\operatorname{Hom}_{\left(\mathcal{H}_{P}^{-}, j_{P}^{-}\right)}(\mathcal{H}, \cdot)$. The other inductions are described by $I_{P}$ or $I_{P}^{\prime}$ (Proposition [4.13). As a corollary, we get a new description of $I_{P}$ (Corollary 4.19).

The functor $I_{P}$ has the left and right adjoint functors which are denoted by $L_{P}$ and $R_{P}$, respectively. We calculate the image of simple modules of

2010 Mathematics Subject Classification. 20C08, 20G25. 
$\mathcal{H}$ by these functors. As proved in [Abe], any simple modules are got in the following way: starting with a simple supersingular module, take the generalized Steinberg module and apply the parabolic induction. So to calculate the image of simple modules of $\mathcal{H}$ by these functors, it is sufficient to calculate the images of parabolic induction, generalized Steinberg modules and supersingular modules. We give such descriptions (Proposition 5.1, Corollary [5.8, Proposition 5.10, Proposition 5.11, Proposition 5.18). In particular, the image of simple modules by these functors is zero or again irreducible (Theorem 5.20). The same calculations for the representations of the group is given in $\mathrm{AHVa}$.

1.2. Applications. The main application is the calculation of the extension groups between simple $\mathcal{H}$-modules. Since the left adjoint functor $L_{P}$ is exact, we have $\operatorname{Ext}^{i}\left(\pi, I_{P}(\sigma)\right) \simeq \operatorname{Ext}^{i}\left(L_{P}(\pi), \sigma\right)$ for $\mathcal{H}$-module $\pi$ and $\mathcal{H}_{P}$-module $\sigma$. We also have a similar formula for the functor $R_{P}$. Since any simple $\mathcal{H}$ module is given by the parabolic induction from the generalized Steinberg modules, these formulas and results in this paper enable us to deduce the calculation of the extension groups to that of the extension groups between generalized Steinberg modules.

For the extension groups between the generalized Steinberg modules, it turns out that we need to study the functor $I_{P}^{\prime}$. Especially, we need the relations between the functors $I_{P}$ and $I_{P}^{\prime}$. Such analysis and the calculation of extension groups will be appeared in sequel in which we use results in this paper.

1.3. Organization of the paper. In the next section we give notation and recall some results mainly from Vig16. In Section 3, we define a certain filtration on $I_{P}(\sigma)$. This filtration is analogous of the filtration of the parabolic induction of the group representation coming from the Bruhat cell. Some properties of the filtration are proved in AHHV, AHVa in the group case and we give analogous of them. These properties is used in Section 5. The study of four "inductions" is done in Section 4. In Section 5. we calculate the image of parabolic induction, generalized Steinberg modules and supersingular modules by $L_{P}$ and $R_{P}$.

Acknowledgments. I thank Guy Henniart and Marie-France Vignéras for helpful discussions. Most of this work was done during my pleasant stay at Institut de mathématiques de Jussieu. The work is supported by JSPS KAKENHI Grant Number 26707001.

\section{Preliminaries}

2.1. Pro- $p$-Iwahori Hecke algebra. Let $\mathcal{H}$ be a pro- $p$-Iwahori Hecke algebra over a commutative ring $C$ [Vig16]. We study modules over $\mathcal{H}$ in this paper. In this paper, a module means a right module. The algebra $\mathcal{H}$ is defined with a combinatorial data $\left(W_{\text {aff }}, S_{\text {aff }}, \Omega, W, W(1), Z_{\kappa}\right)$ and a parameter $(q, c)$.

We recall the definitions. The data satisfy the following.

- $\left(W_{\text {aff }}, S_{\text {aff }}\right)$ is a Coxeter system.

- $\Omega$ acts on ( $\left.W_{\text {aff }}, S_{\text {aff }}\right)$. 
- $W=W_{\text {aff }} \rtimes \Omega$.

- $Z_{\kappa}$ is a finite commutative group.

- The group $W(1)$ is an extension of $W$ by $Z_{\kappa}$, namely we have an exact sequence $1 \rightarrow Z_{\kappa} \rightarrow W(1) \rightarrow W \rightarrow 1$.

The subgroup $Z_{\kappa}$ is normal in $W(1)$. Hence the conjugate action of $w \in$ $W(1)$ induces an automorphism of $Z_{\kappa}$, hence of the group ring $C\left[Z_{\kappa}\right]$. We denote it by $c \mapsto w \cdot c$.

Let $\operatorname{Ref}\left(W_{\text {aff }}\right)$ be the set of reflections in $W_{\text {aff }}$ and $\operatorname{Ref}\left(W_{\text {aff }}(1)\right)$ the inverse image of $\operatorname{Ref}\left(W_{\text {aff }}\right)$ in $W(1)$. The parameter $(q, c)$ is maps $q: S_{\text {aff }} \rightarrow C$ and $c: \operatorname{Ref}\left(W_{\text {aff }}(1)\right) \rightarrow C\left[Z_{\kappa}\right]$ with the following conditions. (Here the image of $s$ by $q$ (resp. $c)$ is denoted by $q_{s}$ (resp. $\left.c_{s}\right)$.)

- For $w \in W$ and $s \in S_{\text {aff }}$, if $w s w^{-1} \in S_{\text {aff }}$ then $q_{w s w^{-1}}=q_{s}$.

- For $w \in W(1)$ and $s \in \operatorname{Ref}\left(W_{\mathrm{aff}}(1)\right), c_{w s w^{-1}}=w \cdot c_{s}$.

- For $s \in \operatorname{Ref}\left(W_{\text {aff }}(1)\right)$ and $t \in Z_{\kappa}$, we have $c_{t s}=t c_{s}$.

Let $S_{\text {aff }}(1)$ be the inverse image of $S_{\text {aff }}$ in $W(1)$. For $s \in S_{\text {aff }}(1)$, we write $q_{s}$ for $q_{\bar{s}}$ where $\bar{s} \in S_{\text {aff }}$ is the image of $s$. The length function on $W_{\text {aff }}$ is denoted by $\ell$ and its inflation to $W$ and $W(1)$ is also denoted by $\ell$.

The $C$-algebra $\mathcal{H}$ is a free $C$-module and has a basis $\left\{T_{w}\right\}_{w \in W(1)}$. The multiplication is given by

- (Quadratic relations) $T_{s}^{2}=q_{s} T_{s^{2}}+c_{s} T_{s}$ for $s \in S_{\text {aff }}(1)$.

- (Braid relations) $T_{v w}=T_{v} T_{w}$ if $\ell(v w)=\ell(v)+\ell(w)$.

We extend $q: S_{\text {aff }} \rightarrow C$ to $q: W \rightarrow C$ as follows. For $w \in W$, take $s_{1}, \ldots, s_{l}$ and $u \in \Omega$ such that $w=s_{1} \cdots s_{l} u$. Then put $q_{w}=q_{s_{1}} \cdots q_{s_{l}}$. From the definition, we have $q_{w^{-1}}=q_{w}$. We also put $q_{w}=q_{\bar{w}}$ for $w \in W(1)$ with the image $\bar{w}$ in $W$.

2.2. The data from a group. Let $F$ be a non-archimedean local field, $\kappa$ its residue field, $p$ its residue characteristic and $G$ a connected reductive group over $F$. We can get the data in the previous subsection from $G$ as follows. See Vig16, especially 3.9 and 4.2 for the details.

Fix a maximal split torus $S$ and denote the centralizer of $S$ by $Z$. Let $Z^{0}$ be the unique parahoric subgroup of $Z$ and $Z(1)$ its pro- $p$ radical. Then the group $W(1)($ resp. $W)$ is defined by $W(1)=N_{G}(Z) / Z(1)\left(W=N_{G}(Z) / Z^{0}\right)$ where $N_{G}(Z)$ is the normalizer of $Z$ in $G$. We also have $Z_{\kappa}=Z^{0} / Z(1)$. Let $G^{\prime}$ be the group generated by the unipotent radical of parabolic subgroups [AHHV, II.1] and $W_{\text {aff }}$ the image of $G^{\prime} \cap N_{G}(Z)$ in $W$. Then this is a Coxeter group. Fix a set of simple reflections $S_{\text {aff }}$. The group $W$ has the natural length function and let $\Omega$ be the set of length zero elements in $W$. Then we get the data ( $\left.W_{\text {aff }}, S_{\text {aff }}, \Omega, W, W(1), Z_{\kappa}\right)$.

Consider the apartment attached to $S$ and an alcove surrounded by the hyperplanes fixed by $S_{\text {aff }}$. Let $I(1)$ be the pro- $p$-Iwahori subgroup attached to this alcove. Then with $q_{s}=\#(I(1) \widetilde{s} I(1) / I(1))$ for $s \in S_{\text {aff }}$ with a lift $\widetilde{s} \in N_{G}(Z)$ and suitable $c_{s}$, the algebra $\mathcal{H}$ is isomorphic to the Hecke algebra attached to $(G, I(1))$ [Vig16, Proposition 4.4].

In this paper, the data $\left(W_{\mathrm{aff}}, S_{\mathrm{aff}}, \Omega, W, W(1), Z_{\kappa}\right)$ and the parameters $(q, c)$ come from $G$ in this way. Let $W_{\text {aff }}(1)$ be the image of $G^{\prime} \cap N_{G}(Z)$ in $W(1)$ and put $\mathcal{H}_{\text {aff }}=\bigoplus_{w \in W_{\text {aff }}(1)} C T_{w}$. This is a subalgebra of $\mathcal{H}$. 
Proposition 2.1 ([Vig16, Proposition 4.4]). Let $s \in \operatorname{Ref}(W(1))$. Then we have $c_{s}=\sum_{t \in Z_{\kappa}} c_{s}(t) T_{t}$ for some $c_{s}(t) \in \mathbb{Z}$ such that $\sum_{t \in Z_{\kappa}} c_{s}(t)=q_{s}-1$.

2.3. Assumptions on $C$. In this paper, $C$ is assumed to be a commutative ring. Sometimes we add the following assumptions.

- $p=0$ in $C$.

- $C$ is an algebraically closed field of characteristic $p$

We declare this assumption at the top of the subsection or the statement of a theorem/proposition/lemma etc. Otherwise we do not assume anything on $C$.

2.4. The algebra $\mathcal{H}\left[q_{s}\right]$ and $\mathcal{H}\left[q_{s}^{ \pm 1}\right]$. For each $s \in S_{\text {aff }}$, let $\mathbf{q}_{s}$ be an indeterminate such that if $w s w^{-1} \in S_{\text {aff }}$ for $w \in W$, we have $\mathbf{q}_{w s w^{-1}}=\mathbf{q}_{s}$. Let $C\left[\mathbf{q}_{s}\right]$ be a polynomial ring with these indeterminate. Then with the parameter $s \mapsto \mathbf{q}_{s}$ and the other data coming from $G$, we have the algebra. This algebra is denoted by $\mathcal{H}\left[\mathbf{q}_{s}\right]$ and we put $\mathcal{H}\left[\mathbf{q}_{s}^{ \pm 1}\right]=\mathcal{H}\left[\mathbf{q}_{s}\right] \otimes_{C\left[\mathbf{q}_{s}\right]} C\left[\mathbf{q}_{s}^{ \pm 1}\right]$. Under $\mathbf{q}_{s} \mapsto \#(I(1) \widetilde{s} I(1) / I(1)) \in C$ where $\widetilde{s} \in N_{G}(Z)$ is a lift of $s$, we have $\mathcal{H}\left[\mathbf{q}_{s}\right] \otimes_{C\left[\mathbf{q}_{s}\right]} C \simeq \mathcal{H}$. As an abbreviation, we denote $\mathbf{q}_{s}$ by just $q_{s}$. Consequently we denote by $\mathcal{H}\left[q_{s}\right]$ (resp. $\mathcal{H}\left[q_{s}^{ \pm 1}\right]$ ).

Since $q_{s}$ is invertible in $\mathcal{H}\left[q_{s}^{ \pm 1}\right]$, we can do some calculations in $\mathcal{H}\left[q_{s}^{ \pm 1}\right]$ with $q_{s}^{-1}$. If the result can be stated in $\mathcal{H}\left[q_{s}\right]$, then this is an equality in $\mathcal{H}\left[q_{s}\right]$ since $\mathcal{H}\left[q_{s}\right]$ is a subalgebra of $\mathcal{H}\left[q_{s}^{ \pm 1}\right]$ and by specializing, we can get some equality in $\mathcal{H}$. See Vig16, 4.5] for more details.

2.5. The root system and the Weyl groups. Let $W_{0}=N_{G}(Z) / Z$ be the finite Weyl group. Then this is a quotient of $W$. Recall that we have the alcove defining $I(1)$. Fix a special point $\boldsymbol{x}_{0}$ from the border of this alcove. Then $W_{0} \simeq \operatorname{Stab}_{W} \boldsymbol{x}_{0}$ and the inclusion $\operatorname{Stab}_{W} \boldsymbol{x}_{0} \hookrightarrow W$ is a splitting of the canonical projection $W \rightarrow W_{0}$. Throughout this paper, we fix this special point and regard $W_{0}$ as a subgroup of $W$. Set $S_{0}=S_{\text {aff }} \cap W_{0} \subset W$. This is a set of simple reflections in $W_{0}$. For each $w \in W_{0}$, we fix a representative $n_{w} \in W(1)$ such that $n_{w_{1} w_{2}}=n_{w_{1}} n_{w_{2}}$ if $\ell\left(w_{1} w_{2}\right)=\ell\left(w_{1}\right)+\ell\left(w_{2}\right)$.

The group $W_{0}$ is the Weyl group of the root system $\Sigma$ attached to $(G, S)$. Our fixed alcove and special point give a positive system of $\Sigma$, denoted by $\Sigma^{+}$. The set of simple roots is denoted by $\Delta$. As usual, for $\alpha \in \Delta$, let $s_{\alpha} \in S_{0}$ be a simple reflection for $\alpha$.

The kernel of $W(1) \rightarrow W_{0}$ (resp. $W \rightarrow W_{0}$ ) is denoted by $\Lambda(1)$ (resp. $\Lambda$ ). Then $Z_{\kappa} \subset \Lambda(1)$ and we have $\Lambda=\Lambda(1) / Z_{\kappa}$. The group $\Lambda$ (resp. $\left.\Lambda(1)\right)$ is isomorphic to $Z / Z^{0}$ (resp. $Z / Z(1)$ ). Any element in $W(1)$ can be uniquely written as $n_{w} \lambda$ where $w \in W_{0}$ and $\lambda \in \Lambda(1)$. We have $W=W_{0} \ltimes \Lambda$.

2.6. The map $\nu$. The group $W$ acts on the apartment attached to $S$ and the action of $\Lambda$ is by the translation. Since the group of translations of the apartment is $X_{*}(S) \otimes_{\mathbb{Z}} \mathbb{R}$, we have a group homomorphism $\nu: \Lambda \rightarrow$ $X_{*}(S) \otimes_{\mathbb{Z}} \mathbb{R}$. The compositions $\Lambda(1) \rightarrow \Lambda \rightarrow X_{*}(S) \otimes_{\mathbb{Z}} \mathbb{R}$ and $Z \rightarrow \Lambda \rightarrow$ $X_{*}(S) \otimes_{\mathbb{Z}} \mathbb{R}$ are also denoted by $\nu$. The homomorphism $\nu: Z \rightarrow X_{*}(S) \otimes_{\mathbb{Z}}$ $\mathbb{R} \simeq \operatorname{Hom}_{\mathbb{Z}}\left(X^{*}(S), \mathbb{R}\right)$ is characterized by the following: For $t \in S$ and $\chi \in X^{*}(S)$, we have $\nu(t)(\chi)=-\operatorname{val}(\chi(t))$ where val is the normalized valuation of $F$. The kernel of $\nu: Z \rightarrow X_{*}(S) \otimes_{\mathbb{Z}} \mathbb{R}$ is equal to the maximal 
compact subgroup $\widetilde{Z}$ of $Z$. In particular, $\operatorname{Ker}\left(\Lambda(1) \stackrel{\nu}{\rightarrow} X_{*}(S) \otimes_{\mathbb{Z}} \mathbb{R}\right)=\widetilde{Z} / Z(1)$ is a finite group.

We call $\lambda \in \Lambda(1)$ dominant (resp. anti-dominant) if $\nu(\lambda)$ is dominant (resp. anti-dominant).

Since the group $W_{\text {aff }}$ is a Coxeter system, it has the Bruhat order denoted by $\leq$. For $w_{1}, w_{2} \in W_{\text {aff }}$, we write $w_{1}<w_{2}$ if there exists $u \in \Omega$ such that $w_{1} u, w_{2} u \in W_{\text {aff }}$ and $w_{1} u<w_{2} u$. Moreover, for $w_{1}, w_{2} \in W(1)$, we write $w_{1}<w_{2}$ if $w_{1} \in W_{\text {aff }}(1) w_{2}$ and $\bar{w}_{1}<\bar{w}_{2}$ where $\bar{w}_{1}, \bar{w}_{2}$ are the image of $w_{1}, w_{2}$ in $W$, respectively. We write $w_{1} \leq w_{2}$ if $w_{1}<w_{2}$ or $w_{1}=w_{2}$.

2.7. Other basis. For $w \in W(1)$, take $s_{1}, \cdots, s_{l} \in S_{\text {aff }}(1)$ and $u \in W(1)$ such that $l=\ell(w), \ell(u)=0$ and $w=s_{1} \cdots s_{l} u$. Set $T_{w}^{*}=\left(T_{s_{1}}-c_{s_{1}}\right) \cdots\left(T_{s_{l}}-\right.$ $\left.c_{s_{l}}\right) T_{u}$. Then this does not depend on the choice and we have $T_{w}^{*} \in T_{w}+$ $\sum_{v<w} C T_{v}$. In particular, $\left\{T_{w}^{*}\right\}_{w \in W(1)}$ is a basis of $\mathcal{H}$. We also have the following other definition of $T_{w}^{*}$. In $\mathcal{H}\left[q_{s}^{ \pm 1}\right], T_{w}$ is invertible and $q_{w} T_{w^{-1}}^{-1} \in$ $\mathcal{H}\left[q_{s}\right]$. The element $T_{w}^{*}$ is the image of $q_{w} T_{w^{-1}}^{-1}$ in $\mathcal{H}$.

For simplicity, we always assume that our commutative ring $C$ contains a square root of $q_{s}$ which is denoted by $q_{s}^{1 / 2}$ for $s \in S_{\text {aff }}$. For $w=s_{1} \cdots s_{l} u$ where $\ell(w)=l$ and $\ell(u)=0, q_{w}^{1 / 2}=q_{s_{1}}^{1 / 2} \cdots q_{s_{l}}^{1 / 2}$ is a square root of $q_{w}$. For a spherical orientation $o$, there is a basis $\left\{E_{o}(w)\right\}_{w \in W(1)}$ of $\mathcal{H}$ introduced in Vig16, 5]. We have

$$
E_{o}(w) \in T_{w}+\sum_{v<w} C T_{v}
$$

This satisfies the following product formula [Vig16, Theorem 5.25].

$$
E_{o}\left(w_{1}\right) E_{o \cdot w_{1}}\left(w_{2}\right)=q_{w_{1} w_{2}}^{-1 / 2} q_{w_{1}}^{1 / 2} q_{w_{2}}^{1 / 2} E_{o}\left(w_{1} w_{2}\right) .
$$

Remark 2.2. Since we do not assume that $q_{s}$ is invertible in $C, q_{w_{1} w_{2}}^{-1 / 2} q_{w_{1}}^{1 / 2} q_{w_{2}}^{1 / 2}$ does not make sense in a usual way. The meaning of this term is the following. Consider the indeterminate $\mathbf{q}_{s}$ as in subsection 2.4. We also add $\mathbf{q}_{s}^{ \pm 1 / 2}$. Then we have $\mathbf{q}_{w_{1} w_{2}}^{-1 / 2} \mathbf{q}_{w_{1}}^{1 / 2} \mathbf{q}_{w_{2}}^{1 / 2}$. Then this belongs to $C\left[\mathbf{q}_{s}\right]$ Vig16, Lemma 4.19]. Hence by specializing $\mathbf{q}_{s} \mapsto q_{s}$, we get $q_{w_{1} w_{2}}^{-1 / 2} q_{w_{1}}^{1 / 2} q_{w_{2}}^{1 / 2} \in$ $C$. Such calculations will be used several times in this paper implicitly. Note that since $\mathbf{q}_{w_{1} w_{2}}^{-1 / 2} \mathbf{q}_{w_{1}}^{1 / 2} \mathbf{q}_{w_{2}}^{1 / 2}$ is in $C\left[\mathbf{q}_{s}\right]$, rather than $C\left[\mathbf{q}_{s}^{1 / 2}\right]$, the factor $q_{w_{1} w_{2}}^{-1 / 2} q_{w_{1}}^{1 / 2} q_{w_{2}}^{1 / 2}$ does not depend on a choice of the square roots.

Applying (2.1) to $w_{1}=w^{-1}$ and $w_{2}=w$, if $q_{w}$ is invertible then $E_{o \cdot w}(w)$ is invertible and we have

$$
E_{o}\left(w^{-1}\right)=q_{w} E_{o \cdot w}(w)^{-1} .
$$

There is a one-to-one correspondence between spherical orientations and closed Weyl chambers. Let $o$ be a spherical orientation and $\mathcal{D}$ the corresponding closed Weyl chamber. Then for $\lambda \in \Lambda(1)$, we have [Vig16, Example 5.30]

$$
E_{o}(\lambda)= \begin{cases}T_{\lambda} & (\nu(\lambda) \in \mathcal{D}), \\ T_{\lambda}^{*} & (-\nu(\lambda) \in \mathcal{D}) .\end{cases}
$$

Let $o_{+}$(resp. $o_{-}$) be the dominant (resp. anti-dominant) orientation. The orientation $o_{+}$(resp. $o_{-}$) corresponds to the dominant (resp. anti-dominant) 
chamber. Then for $s \in S_{0}$ and $v \in W_{0}$ [Vig16, Example 5.33],

$$
E_{o_{+} \cdot v}\left(n_{s}\right)=\left\{\begin{array}{ll}
T_{n_{s}} & (v s<v), \\
T_{n_{s}}^{*} & (v s>v),
\end{array} \quad E_{o_{-} \cdot v}\left(n_{s}\right)= \begin{cases}T_{n_{s}} & (v s>v), \\
T_{n_{s}}^{*} & (v s<v) .\end{cases}\right.
$$

By the definition of spherical orientations, $o \cdot \lambda=o$ for any spherical orientation $o$ and $\lambda \in \Lambda(1)$. Hence by (2.1), the subspace $\bigoplus_{\lambda \in \Lambda(1)} C E_{o}(\lambda)$ is a subalgebra of $\mathcal{H}$. We denote this subalgebra by $\mathcal{A}_{o}$.

Finally we introduce one more basis defined by

$$
E_{-}\left(n_{w} \lambda\right)=q_{n_{w} \lambda}^{1 / 2} q_{n_{w}}^{-1 / 2} q_{\lambda}^{-1 / 2} T_{n_{w}}^{*} E_{o_{-}}(\lambda)
$$

for $w \in W_{0}$ and $\lambda \in \Lambda(1)$. By [Abe, Lemma 4.2], $\left\{E_{-}(w) \mid w \in W(1)\right\}$ is a $C$-basis of $\mathcal{H}$.

2.8. Levi subalgebra. Since we have a positive system $\Sigma^{+}$, we have a minimal parabolic subgroup $B$ with a Levi part $Z$. In this paper, parabolic subgroups are always standard, namely containing $B$. Note that such parabolic subgroups correspond to subsets of $\Delta$.

Let $P$ be a parabolic subgroup. Attached to the Levi part of $P$ containing $Z$, we have a data ( $\left.W_{\mathrm{aff}, P}, S_{\mathrm{aff}, P}, \Omega_{P}, W_{P}, W_{P}(1), Z_{\kappa}\right)$ and parameters $\left(q_{P}, c_{P}\right)$. Hence we have the algebra $\mathcal{H}$. The parameter $c_{P}$ is given by the restriction of $c$, hence we denote it just by $c$. The parameter $q_{P}$ is defined as in Abe, 4.1].

For the objects attached to this data, we add a suffix $P$. We have the set of simple roots $\Delta_{P}$, the root system $\Sigma_{P}$ and its positive system $\Sigma_{P}^{+}$, the finite Weyl group $W_{0, P}$, the set of simple reflections $S_{0, P} \subset W_{0, P}$, the length function $\ell_{P}$ and the base $\left\{T_{w}^{P}\right\}_{w \in W_{P}(1)},\left\{T_{w}^{P *}\right\}_{w \in W_{P}(1)}$ and $\left\{E_{o}^{P}(w)\right\}_{w \in W_{P}(1)}$ of $\mathcal{H}_{P}$. Note that we have no $\Lambda_{P}, \Lambda_{P}(1)$ and $Z_{\kappa, P}$ since they are equal to $\Lambda, \Lambda(1)$ and $Z_{\kappa}$. We have the following lemma. This follows from Vig16, $3.8,4.2]$. See also [AHVb, Remark 4.6].

Lemma 2.3. Let $s \in S_{\text {aff }}, \widetilde{s} \in S_{\text {aff }}(1)$ its lift, $\alpha \in \Sigma$ such that the image of $s$ in $W_{0}$ is $s_{\alpha}$ and $P$ a parabolic subgroup such that $\alpha \in \Sigma_{P}$.

(1) We can take $\widetilde{s}$ from $W_{\text {aff }, P}(1)$.

(2) If $\widetilde{s} \in W_{\mathrm{aff}, P}(1)$, then $c_{\tilde{s}} \in C\left[Z_{\kappa} \cap W_{\mathrm{aff}, P}(1)\right]$.

In particular, for $s=s_{\alpha} \in S_{0}$ where $\alpha \in \Delta$, we can take (and do) $n_{s} \in W_{\text {aff }, P_{\alpha}}(1)$ where $P_{\alpha}$ is a parabolic subgroup such that $\Delta_{P_{\alpha}}=\{\alpha\}$.

An element $w=n_{v} \lambda \in W_{P}(1)$ where $v \in W_{0, P}$ and $\lambda \in \Lambda(1)$ is called $P$-positive (resp. $P$-negative) if $\langle\alpha, \nu(\lambda)\rangle \leq 0$ (resp. $\langle\alpha, \nu(\lambda)\rangle \geq 0$ ) for any $\alpha \in \Sigma^{+} \backslash \Sigma_{P}^{+}$. Let $W_{P}^{+}(1)$ (resp. $W_{P}^{-}(1)$ ) be the set of $P$-positive (resp. $P$ negative) elements and put $\mathcal{H}_{P}^{ \pm}=\bigoplus_{w \in W_{P}^{ \pm}(1)} C T_{w}^{P}$. These are subalgebras of $\mathcal{H}_{P}$ [Abe, Lemma 4.1]. In general, for a group $\Gamma$, we denote its center by $Z(\Gamma)$.

Lemma 2.4. There exists $\lambda_{P}^{+}$(resp. $\left.\lambda_{P}^{-}\right)$in the center of $W_{P}(1)$ such that $\left\langle\alpha, \nu\left(\lambda_{P}^{+}\right)\right\rangle<0$ (resp. $\left\langle\alpha, \nu\left(\lambda_{P}^{-}\right)\right\rangle>0$ ) for all $\alpha \in \Sigma^{+} \backslash \Sigma_{P}^{+}$.

Proof. We prove the existence of $\lambda_{P}^{+}$. Take $\lambda_{0} \in Z(\Lambda(1))$ such that the stabilizers of $\nu\left(\lambda_{0}\right)$ in $W_{0}$ is $W_{0, P}$ and $\left\langle\alpha, \nu\left(\lambda_{0}\right)\right\rangle<0$ for any $\alpha \in \Sigma^{+} \backslash \Sigma_{P}^{+}$. Fix $w \in W_{0, P}$. Then for each $k \in \mathbb{Z}_{\geq 0}, \nu\left(n_{w} \lambda_{0}^{k} n_{w}^{-1} \lambda_{0}^{-k}\right)=0$. Namely 
$n_{w} \lambda_{0}^{k} n_{w}^{-1} \lambda_{0}^{-k} \in \operatorname{Ker} \nu$. Since $\operatorname{Ker} \nu$ is finite, there exists $k_{1}>k_{2}$ such that $n_{w} \lambda_{0}^{k_{1}} n_{w}^{-1} \lambda_{0}^{-k_{1}}=n_{w} \lambda_{0}^{k_{2}} n_{w}^{-1} \lambda_{0}^{-k_{2}}$. Hence $n_{w} \lambda_{0}^{k_{1}-k_{2}} n_{w}^{-1} \lambda_{0}^{-\left(k_{1}-k_{2}\right)}=1$. Therefore, for each $w \in W_{0, P}$, there exists $k_{w} \in \mathbb{Z}_{>0}$ such that $n_{w} \lambda_{0}^{k_{w}} n_{w}^{-1} \lambda_{0}^{-k_{w}}=1$. Hence $n_{w} \cdot \lambda_{0}^{k_{w}}=\lambda_{0}^{k_{w}}$. Set $k=\prod_{w \in W_{0, P}} k_{w}$ and $\lambda=\lambda_{0}^{k}$. Then for $w \in W_{0, P}$, we have $n_{w} \cdot \lambda=\lambda$. Since we took $\lambda_{0}$ from the center of $\Lambda(1)$, $\lambda$ commutes with the elements in $\Lambda(1)$. The group $W_{P}(1)$ is generated by $\left\{n_{w} \mid w \in W_{0, P}\right\}$ and $\Lambda(1)$. Hence $\lambda \in Z\left(W_{P}(1)\right)$. For $\alpha \in \Sigma^{+} \backslash \Sigma_{P}^{+}$, we have $\langle\alpha, \nu(\lambda)\rangle=k\left\langle\alpha, \nu\left(\lambda_{0}\right)\right\rangle<0$. Therefore $\lambda_{P}^{+}=\lambda$ satisfies the condition of the lemma. The element $\lambda_{P}^{-}=\left(\lambda_{P}^{+}\right)^{-1}$ satisfies the condition of the lemma.

Proposition 2.5 ( $\left[\operatorname{Vig} 15 \mathrm{~b}\right.$, Theorem 1.4]). Let $\lambda_{P}^{+}$(resp. $\left.\lambda_{P}^{-}\right)$be in the center of $W_{P}(1)$ such that $\left\langle\alpha, \nu\left(\lambda_{P}^{+}\right)\right\rangle<0$ (resp. $\left\langle\alpha, \nu\left(\lambda_{P}^{-}\right)\right\rangle>0$ ) for all $\alpha \in \Sigma^{+} \backslash \Sigma_{P}^{+}$. Then $T_{\lambda_{P}^{+}}^{P}=T_{\lambda_{P}^{+*}}^{P *}=E_{o_{-, P}}^{P}\left(\lambda_{P}^{+}\right)\left(\right.$resp. $\left.T_{\lambda_{P}^{-}}^{P}=T_{\lambda_{P}^{-}}^{P *}=E_{o_{-, P}}^{P}\left(\lambda_{P}^{-}\right)\right)$ is in the center of $\mathcal{H}_{P}$ and we have $\mathcal{H}_{P}=\mathcal{H}_{P}^{+} E_{o_{-}, P}^{P}\left(\lambda_{P}^{+}\right)^{-1}$ (resp. $\mathcal{H}_{P}=$ $\left.\mathcal{H}_{P}^{-} E_{o_{-, P}}^{P}\left(\lambda_{P}^{-}\right)^{-1}\right)$.

We define $j_{P}^{ \pm}: \mathcal{H}_{P}^{ \pm} \rightarrow \mathcal{H}$ and $j_{P}^{ \pm *}: \mathcal{H}_{P}^{ \pm} \rightarrow \mathcal{H}$ by $j_{P}^{ \pm}\left(T_{w}^{P}\right)=T_{w}$ and $j_{P}^{ \pm *}\left(T_{w}^{P *}\right)=T_{w}^{*}$ for $w \in W_{P}^{ \pm}(1)$. Then these are algebra homomorphisms. For $j_{P}^{+}$and $j_{P}^{-*}$, it is [Abe, Lemma 4.6] and the same argument can apply for $j_{P}^{+*}$ and $j_{P}^{-}$.

Some special cases of the following lemma is proved in Oll15, Abe].

Lemma 2.6. Let $w \in W_{P}(1)$.

(1) If the image of $w$ in $W_{P}$ is in $W_{0, P}$, then $w$ is both $P$-positive and $P$-negative. Moreover we have $j_{P}^{ \pm}\left(T_{w}^{P *}\right)=T_{w}^{*}$ and $j_{P}^{ \pm *}\left(T_{w}^{P}\right)=T_{w}$.

(2) For $x \in W_{0, P}$, we have

$$
\begin{aligned}
j_{P}^{+}\left(E_{o_{-, P} \cdot x}^{P}(w)\right) & =E_{o_{-} \cdot x}(w) & & \left(w \in W_{P}^{+}(1)\right), \\
j_{P}^{+*}\left(E_{o_{+}, P}^{P}(w)\right) & =E_{o_{+} \cdot x}(w) & & \left(w \in W_{P}^{+}(1)\right), \\
j_{P}^{-}\left(E_{o_{+}, P}^{P}(w)\right) & =E_{o_{+} \cdot x}(w) & & \left(w \in W_{P}^{-}(1)\right), \\
j_{P}^{-*}\left(E_{o_{-}, P}^{P}(w)\right) & =E_{o_{-} \cdot x}(w) & & \left(w \in W_{P}^{-}(1)\right) .
\end{aligned}
$$

Proof. (1) Let $v$ be the image of $w$ in $W_{P}$. Then it is in $W_{0, P}$ by the assumption. Set $t=n_{v}^{-1} w$. Then $t \in \operatorname{Ker}\left(W_{P}(1) \rightarrow W_{P}\right)=Z_{\kappa}$. In particular $t \in \Lambda(1)$ and we have $w=n_{v} t$. Since $\nu(t)=0, w$ is $P$-positive and $P$-negative.

For the proof of the second statement, first assume that $w=t \in Z_{\kappa}$. Since $\ell(t)=0$, we have $T_{t}^{P}=T_{t}^{P *}$ and $T_{t}=T_{t}^{*}$. Therefore we get the statement. In particular, for $s \in S_{\text {aff }}(1)$, we have $j_{P}^{ \pm}\left(c_{s}\right)=j_{P}^{ \pm *}\left(c_{s}\right)=c_{s}$.

To prove the second statement, by induction on $\ell_{P}(w)$, we may assume that the image of $w$ in $W_{0, P}$ is a simple reflection. Set $s=w$. Then $j_{P}^{ \pm}\left(T_{s}^{P *}\right)=j_{P}^{ \pm}\left(T_{s}^{P}-c_{s}\right)=T_{s}-c_{s}$. Since the image of $s$ in $W_{P}$ is a finite simple reflection, the image of $s$ in $W$ is also a finite simple reflection. Therefore $T_{s}^{*}=T_{s}-c_{s}$. Hence $j_{P}^{ \pm}\left(T_{s}^{P *}\right)=T_{s}^{*}$. Similarly we have $j_{P}^{ \pm *}\left(T_{s}^{P}\right)=$ $j_{P}^{ \pm *}\left(T_{s}^{P *}+c_{s}\right)=T_{s}^{*}+c_{s}=T_{s}$.

(2) It is sufficient to prove the lemma in $\mathcal{H}\left[q_{s}^{ \pm 1}\right]$. First we prove the following: Let $w_{1}, w_{2}, w_{3}$ be $P$-negative or $P$-positive elements such that 
$w_{3}=w_{1} w_{2}$. Then if the lemma holds for $w=w_{1}, w_{2}$ (resp. $w=w_{2}, w_{3}$ ) then it also holds for $w=w_{3}$ (resp. $w=w_{1}$ ). We prove this claim only for $j_{P}^{+}$. For the other cases, the same proof apply.

For $j_{P}^{+}$, we assume that $w_{1}, w_{2}, w_{3}$ are $P$-positive. By (2.1), we have

$$
E_{o_{-, P} \cdot x}^{P}\left(w_{1}\right) E_{o_{-, P} \cdot n_{x} w_{1}}^{P}\left(w_{2}\right)=q_{P, w_{1}}^{1 / 2} q_{P, w_{2}}^{1 / 2} q_{P, w_{3}}^{-1 / 2} E_{o_{-, P} \cdot x}^{P}\left(w_{3}\right) .
$$

Since $j_{P}^{+}$is an algebra homomorphism, we have

$$
j_{P}^{+}\left(E_{o_{-, P} \cdot x}^{P}\left(w_{1}\right)\right) j_{P}^{+}\left(E_{o_{-, P} \cdot n_{x} w_{1}}^{P}\left(w_{2}\right)\right)=q_{P, w_{1}}^{1 / 2} q_{P, w_{2}}^{1 / 2} q_{P, w_{3}}^{-1 / 2} j_{P}^{+}\left(E_{o_{-, P} \cdot x}^{P}\left(w_{3}\right)\right) .
$$

By [Abe, Lemma 4.5], we have $q_{P, w_{1}}^{1 / 2} q_{P, w_{2}}^{1 / 2} q_{P, w_{3}}^{-1 / 2}=q_{w_{1}}^{1 / 2} q_{w_{2}}^{1 / 2} q_{w_{3}}^{-1 / 2}$. Hence

$$
j_{P}^{+}\left(E_{o_{-, P} \cdot x}^{P}\left(w_{1}\right)\right) j_{P}^{+}\left(E_{o_{-, P} \cdot n_{x} w_{1}}^{P}\left(w_{2}\right)\right)=q_{w_{1}}^{1 / 2} q_{w_{2}}^{1 / 2} q_{w_{3}}^{-1 / 2} j_{P}^{+}\left(E_{o_{-, P} \cdot x}^{P}\left(w_{3}\right)\right) .
$$

If the lemma holds for $w=w_{1}$ and $w_{2}$, then

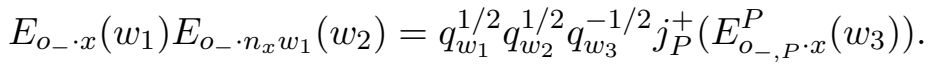

By (2.1), we get $j_{P}^{+}\left(E_{o_{-}, P}^{P}\left(w_{3}\right)\right)=E_{o_{-} \cdot x}\left(w_{3}\right)$. If the lemma holds for $w=w_{2}$ and $w_{3}$, then

$$
j_{P}^{+}\left(E_{o_{-}, P}^{P}\left(w_{1}\right)\right) E_{o_{-} \cdot n_{x} w_{1}}\left(w_{2}\right)=q_{w_{1}}^{1 / 2} q_{w_{2}}^{1 / 2} q_{w_{3}}^{-1 / 2} E_{o_{-} \cdot x}\left(w_{3}\right) .
$$

By (2.2),$E_{O_{-} P \cdot n_{x} w_{1}}\left(w_{2}\right)$ is invertible in $\mathcal{H}\left[q_{s}^{ \pm 1}\right]$. Hence by (2.1), we get $j_{P}^{+}\left(E_{o_{-}, P}^{P}\left(w_{1}\right)\right)=E_{o_{-} \cdot x}\left(w_{1}\right)$. The same proof can be applicable for the other cases.

We assume that $w=n_{v}$ for $v \in W_{0, P}$. By the above argument, we may assume that $v \in S_{0, P}$. We write $s$ for $v$. By (2.4), we have

$$
E_{o_{+}, P}^{P}\left(n_{s}\right)=\left\{\begin{array}{ll}
T_{n_{s}}^{P} & (x s<x), \\
T_{n_{s}}^{P *} & (x s>x),
\end{array} \quad E_{o_{-, P} \cdot x}^{P}\left(n_{s}\right)= \begin{cases}T_{n_{s}}^{P} & (x s>x), \\
T_{n_{s}}^{P *} & (x s<x) .\end{cases}\right.
$$

Hence by (1), for $j=j_{P}^{+*}$ or $j_{P}^{-}$and $j^{\prime}=j_{P}^{+}$or $j_{P}^{-*}$, we have

$$
j\left(E_{o_{+, P} \cdot x}^{P}\left(n_{s}\right)\right)=\left\{\begin{array}{ll}
T_{n_{s}} & (x s<x), \\
T_{n_{s}}^{*} & (x s>x),
\end{array} \quad j^{\prime}\left(E_{o_{-, P} \cdot x}^{P}\left(n_{s}\right)\right)= \begin{cases}T_{n_{s}} & (x s>x), \\
T_{n_{s}}^{*} & (x s<x) .\end{cases}\right.
$$

On the other hand, we have

$$
E_{o_{+} \cdot x}\left(n_{s}\right)=\left\{\begin{array}{ll}
T_{n_{s}} & (x s<x), \\
T_{n_{s}}^{*} & (x s>x),
\end{array} \quad E_{o_{-} \cdot x}\left(n_{s}\right)= \begin{cases}T_{n_{s}} & (x s>x), \\
T_{n_{s}}^{*} & (x s<x) .\end{cases}\right.
$$

We get the lemma in this case.

Next we assume that $w=\lambda \in \Lambda(1)$ and it is in a chamber corresponding to the spherical orientation. We deal with 4 cases separately. Note that since $x\left(\Sigma^{+} \backslash \Sigma_{P}^{+}\right)=\Sigma^{+} \backslash \Sigma_{P}^{+}, n_{x} \cdot \lambda$ is $P$-positive (resp. $P$-negative) if and only if $\lambda$ is $P$-positive (reps. $P$-negative).

$j_{P}^{+}$: Assume that $n_{x} \cdot \lambda$ is anti-dominant. Then $n_{x} \cdot \lambda$ is $P$-positive. Hence $\lambda$ is also $P$-positive. We have $\left.E_{o_{-}, P}^{P} \cdot x\right)=T_{\lambda}^{P}$ and $E_{o_{-} \cdot x}(\lambda)=T_{\lambda}$ by (2.3). Hence $j_{P}^{+}\left(E_{o_{-, P} \cdot x}^{P}(\lambda)\right)=E_{o_{-} \cdot x}(\lambda)$ in this case.

$j_{P}^{+*}$ : Assume that $n_{x} \cdot \lambda$ is anti-dominant. Then $n_{x} \cdot \lambda$ is $P$-positive. Hence $\lambda$ is also $P$-positive. We have $E_{o_{+}, x}^{P}(\lambda)=T_{\lambda}^{P *}$ and $E_{o_{+} \cdot x}(\lambda)=T_{\lambda}^{*}$ by (2.3). Hence $j_{P}^{+*}\left(E_{o_{+}, P}^{P}(\lambda)\right)=E_{o_{+} \cdot x}(\lambda)$ in this case. 
$j_{P}^{-}$: Assume that $n_{x} \cdot \lambda$ is dominant. Then $n_{x} \cdot \lambda$ is $P$-negative. Hence $\lambda$ is also $P$-negative. We have $E_{o_{+}, P}^{P}(\lambda)=T_{\lambda}^{P}$ and $E_{o_{+} \cdot x}(\lambda)=T_{\lambda}$ by (2.3). Hence $j_{P}^{-}\left(E_{o_{+}, P}^{P} \cdot x(\lambda)\right)=E_{o_{+} \cdot x}(\lambda)$ in this case.

$j_{P}^{-*}$ : Assume that $n_{x} \cdot \lambda$ is dominant. Then $n_{x} \cdot \lambda$ is $P$-negative. Hence $\lambda$ is also $P$-negative. We have $E_{o_{-} P}^{P \cdot x}(\lambda)=T_{\lambda}^{P *}$ and $E_{o_{-} \cdot x}(\lambda)=T_{\lambda}^{*}$ by (2.3). Hence $j_{P}^{-*}\left(E_{o_{-, P} \cdot x}^{P}(\lambda)\right)=E_{o_{-} \cdot x}(\lambda)$ in this case.

We prove the general case for $j_{P}^{+}$. The same argument implies the other cases. Let $w \in W_{P}^{+}(1)$ and take $v \in W_{0}, \lambda_{1}, \lambda_{2} \in \Lambda(1) \cap W_{P}^{+}(1)$ such that $w=n_{v} \lambda_{1} \lambda_{2}^{-1}, n_{x} \cdot \lambda_{1}$ and $n_{x} \cdot \lambda_{2}$ are anti-dominant. Then the lemma holds for $n_{v}, \lambda_{1}$ and $\lambda_{2}$. Hence the argument in the beginning of the proof of (2), we get the lemma for $w$.

Corollary 2.7. If $w \in W_{P}(1)$ is both $P$-positive and P-negative (in particular, if $w=n_{v}$ for some $\left.v \in W_{0, P}\right)$, then we have $j_{P}^{ \pm}\left(T_{w}^{P *}\right)=T_{w}^{*}$ and $j_{P}^{ \pm *}\left(T_{w}^{P}\right)=T_{w}$.

Proof. We only prove $j_{P}^{+}\left(T_{w}^{P *}\right)=T_{w}^{*}$. The same argument apply to other cases.

Take $c_{v} \in C$ such that $T_{w}^{P *}=\sum_{v} c_{v} E_{o_{-}, P}^{P}(v)$. If $c_{v} \neq 0$ then $v \leq w$ in $W_{P}(1)$. Hence $v$ is also $P$-positive and $P$-negative by [Abe, Lemma 4.1]. We have

$$
\begin{aligned}
j_{P}^{+}\left(T_{w}^{P *}\right) & =\sum_{v} c_{v} j_{P}^{+}\left(E_{o_{-, P}}^{P}(v)\right) \\
& =\sum_{v} c_{v} E_{o_{-}}(v) \\
& =\sum_{v} c_{v} j_{P}^{-*}\left(E_{o_{-, P}}^{P}(v)\right) \\
& =j_{P}^{-*}\left(T_{w}^{P *}\right)=T_{w}^{*} .
\end{aligned}
$$

We get the corollary.

We also use the following relative setting. Let $Q$ be a parabolic subgroup containing $P$ and let $W_{P}^{Q+}(1)$ (resp. $W_{P}^{Q-}(1)$ ) be the set of $n_{w} \lambda$ where $\langle\alpha, \nu(\lambda)\rangle \leq 0(\operatorname{resp} .\langle\alpha, \nu(\lambda)\rangle \geq 0)$ for any $\alpha \in \Sigma_{Q}^{+} \backslash \Sigma_{P}^{+}$and $w \in$ $W_{0, P}$. Put $\mathcal{H}_{P}^{Q \pm}=\bigoplus_{w \in W_{P}^{Q \pm}(1)} C T_{w}^{P} \subset \mathcal{H}_{P}$. Then we have homomorphisms $j_{P}^{Q \pm}, j_{P}^{Q \pm *}: \mathcal{H}_{P}^{Q \pm} \rightarrow \mathcal{H}_{Q}$ defined by a similar way.

2.9. Parabolic induction. Let $P$ be a parabolic subgroup and $\sigma$ an $\mathcal{H}_{P^{-}}$ module. (This is a right module as in subsection [2.1.) Then we define an $\mathcal{H}$-module $I_{P}(\sigma)$ by

$$
I_{P}(\sigma)=\operatorname{Hom}_{\left(\mathcal{H}_{P}^{-}, j_{P}^{-*}\right)}(\mathcal{H}, \sigma) .
$$

We call $I_{P}$ the parabolic induction. For $P \subset P_{1}$, we write

$$
I_{P}^{P_{1}}(\sigma)=\operatorname{Hom}_{\left(\mathcal{H}_{P}^{P_{1}-}, j_{P}^{P_{1}-*}\right)}\left(\mathcal{H}_{P_{1}}, \sigma\right) .
$$

Remark 2.8. Since we have two algebras $\mathcal{H}_{P}^{ \pm}$and four homomorphisms $j_{P}^{ \pm}, j_{P}^{ \pm *}$, we can define four "inductions". The other inductions are studied in Section 4 . 
We recall some properties from $\mathrm{Abe}$ and $\operatorname{Vig} 15 \mathrm{~b}$. Let $P$ be a parabolic subgroup. Set $W_{0}^{P}=\left\{w \in W_{0} \mid w\left(\Delta_{P}\right) \subset \Sigma^{+}\right\}$. Then the multiplication map $W_{0}^{P} \times W_{0, P} \rightarrow W_{0}$ is bijective and for $w_{1} \in W_{0}^{P}$ and $w_{2} \in W_{0, P}$, we have $\ell\left(w_{1} w_{2}\right)=\ell\left(w_{1}\right)+\ell\left(w_{2}\right)$. We also put ${ }^{P} W_{0}=\left\{w \in W_{0} \mid w^{-1}\left(\Delta_{P}\right) \subset\right.$ $\left.\Sigma^{+}\right\}$. Then the multiplication map $W_{0, P} \times{ }^{P} W_{0} \rightarrow W_{0}$ is bijective and for $w_{1} \in W_{0, P}$ and $w_{2} \in{ }^{P} W_{0}$, we have $\ell\left(w_{1} w_{2}\right)=\ell\left(w_{1}\right)+\ell\left(w_{2}\right)$.

Proposition 2.9. Let $P$ be a parabolic subgroup and $\sigma$ an $\mathcal{H}_{P}$-module.

(1) The map $I_{P}(\sigma) \ni \varphi \mapsto\left(\varphi\left(T_{n_{w}}\right)\right)_{w \in W_{0}^{P}} \in \bigoplus_{w \in W_{0}^{P}} \sigma$ is bijective.

(2) The map $I_{P}(\sigma) \ni \varphi \mapsto\left(\varphi\left(T_{n_{w}}^{*}\right)\right)_{w \in W_{0}^{P}} \in \bigoplus_{w \in W_{0}^{P}} \sigma$ is bijective.

Proof. The first part is [Vig15b, Lemma 3.9]. The second part follows from the first part and $T_{n_{w}}^{*} \in T_{n_{w}}+\sum_{v<w} T_{n_{v}} C\left[Z_{\kappa}\right]$ with a usual triangular argument.

Let $P \subset Q$ be parabolic subgroups. We have an $\mathcal{H}_{Q}$-module $I_{P}^{Q}(\sigma)$ for an $\mathcal{H}_{P}$-module $\sigma$. The parabolic inductions are transitive.

Proposition 2.10 (Vig15b, Proposition 4.10]). The map $\varphi \mapsto(X \mapsto$ $\varphi(X)(1))$ gives an isomorphism $I_{Q}\left(I_{P}^{Q}(\sigma)\right) \simeq I_{P}(\sigma)$.

2.10. Length. We prove some lemmas on the length. These lemmas follow from the formula on the length [Vig16.

We recall the formula. Let $\Sigma^{\prime} \subset \operatorname{Hom}_{\mathbb{R}}\left(X_{*}(S) \otimes_{\mathbb{Z}} \mathbb{R}, \mathbb{R}\right)$ be the subset such that the action of $\operatorname{Ref}\left(W_{\text {aff }}\right)$ on $X_{*}(S) \otimes_{\mathbb{Z}} \mathbb{R}$ is the set of reflections with respect to the hyperplanes $\left\{\left\{v \in X_{*}(S) \otimes_{\mathbb{Z}} \mathbb{R} \mid \alpha(v)+k=0\right\} \mid \alpha \in \Sigma^{\prime}, k \in \mathbb{Z}\right\}$. Then for any $\alpha \in \Sigma^{\prime}$, there exists only one element in $\mathbb{R}_{>0} \alpha \cap \Sigma_{\text {red }}$ and the map $\alpha$ sends to this unique element gives a bijection $\Sigma^{\prime} \simeq \Sigma_{\text {red }}$ where $\Sigma_{\text {red }}$ is the set of reduced roots in $\Sigma$. Set $\Sigma^{\prime+}=\left\{\alpha \in \Sigma^{\prime} \mid \Sigma^{+} \cap \mathbb{R}_{>0} \alpha \neq \emptyset\right\}$. Then $\Sigma^{\prime}$ is a root system with the Weyl group $W_{0}$ and $\Sigma^{\prime+}$ is a positive system of $\Sigma^{\prime}$. For $v \in W_{0}$ and $\lambda \in \Lambda(1)$, we have [Vig16, Corollary 5.10]

$$
\begin{aligned}
& \ell\left(\lambda n_{v}\right)=\sum_{\alpha \in \Sigma^{\prime+} \cap v\left(\Sigma^{\prime+}\right)}|\langle\alpha, \nu(\lambda)\rangle|+\sum_{\alpha \in \Sigma^{\prime+} \cap v\left(\Sigma^{\prime}\right)}|\langle\alpha, \nu(\lambda)\rangle-1|, \\
& \ell\left(n_{v} \lambda\right)=\sum_{\alpha \in \Sigma^{\prime+} \cap v^{-1}\left(\Sigma^{\prime+}\right)}|\langle\alpha, \nu(\lambda)\rangle|+\sum_{\alpha \in \Sigma^{\prime+} \cap v^{-1}\left(\Sigma^{\prime-}\right)}|\langle\alpha, \nu(\lambda)\rangle+1| .
\end{aligned}
$$

The map $W \rightarrow \operatorname{Aut}\left(X_{*}(S) \otimes_{\mathbb{Z}} \mathbb{R}\right)$ is injective on $W_{\text {aff }}$ and the image of $W_{\text {aff }}$ is the Weyl group of the affine root system $\left\{\alpha+k \mid \alpha \in \Sigma^{\prime}, k \in \mathbb{Z}\right\}$. Hence we have $W_{\text {aff }} \simeq W_{0} \ltimes \mathbb{Z} \Sigma^{\prime \vee}$. For $\lambda \in \Lambda(1) \cap W_{\text {aff }}(1), \nu(\lambda)$ is in the image of $\Lambda \cap W_{\text {aff. }}$. It is $\mathbb{Z} \Sigma^{\prime \vee}$. Hence $\nu(\lambda) \in \mathbb{Z} \Sigma^{\prime \vee} \subset \mathbb{R} \Sigma^{\vee}$.

Lemma 2.11. Let $\lambda_{1}, \lambda_{2} \in \Lambda(1)$. We have $\ell\left(\lambda_{1} \lambda_{2}\right)=\ell\left(\lambda_{1}\right)+\ell\left(\lambda_{2}\right)$ if and only if the vectors $\nu\left(\lambda_{1}\right), \nu\left(\lambda_{2}\right)$ are in the same closed Weyl chamber, namely $\left\langle\alpha, \nu\left(\lambda_{1}\right)\right\rangle\left\langle\alpha, \nu\left(\lambda_{2}\right)\right\rangle \geq 0$ for any $\alpha \in \Sigma^{+}$.

Proof. Obvious from the above formula.

Lemma 2.12. Let $\lambda \in \Lambda(1)$. Then we have $\ell(\lambda)=0$ if and only if $\langle\alpha, \nu(\lambda)\rangle=0$ for any $\alpha \in \Sigma$.

Proof. Obvious from the above formula. 
Lemma 2.13. If $w \in W(1)$ is in the center of $W(1)$, then $w \in \Lambda(1)$ and $\ell(w)=0$.

Proof. If $w$ is in the center, then $w \in \Lambda(1)$ by [Vig14, Lemma 1.1]. For $\alpha \in \Sigma$, we have $n_{s_{\alpha}} w n_{s_{\alpha}}^{-1}=w$. Applying $\nu$, we get $s_{\alpha}(\nu(w))=\nu(w)$. Hence $\langle\alpha, \nu(w)\rangle=0$.

Lemma 2.14. If $\lambda \in \Lambda(1) \cap W_{\text {aff }}(1)$, then $\ell(\lambda)$ is even.

Proof. We have $\ell(\lambda)=\sum_{\alpha \in \Sigma^{+}}|\langle\alpha, \nu(\lambda)\rangle| \equiv \sum_{\alpha \in \Sigma^{\prime+}}\langle\alpha, \nu(\lambda)\rangle=2\langle\rho, \nu(\lambda)\rangle$ $(\bmod 2)$ where $\rho=(1 / 2) \sum_{\alpha \in \Sigma^{\prime}} \alpha$. For each $\alpha \in \Sigma^{\prime}$ which is simple, $\left\langle\rho, \alpha^{\vee}\right\rangle=1$. Hence for any $v \in \mathbb{Z} \Sigma^{\prime \vee}$, we have $\langle\rho, v\rangle \in \mathbb{Z}$. Hence $2\langle\rho, \nu(\lambda)\rangle$ is even.

Lemma 2.15. For $w \in W(1)$ and $\lambda \in \Lambda(1)$, we have $\ell(w \cdot \lambda)=\ell(\lambda)$.

Proof. Let $x \in W_{0}$ be the image of $w$. Then we have $\nu(w \cdot \lambda)=x(\nu(\lambda))$. Hence $\ell(w \cdot \lambda)=\sum_{\alpha \in \Sigma^{+}}|\langle\alpha, x(\nu(\lambda))\rangle|=\sum_{\alpha \in x^{-1}\left(\Sigma^{+}\right)}|\langle\alpha, \nu(\lambda)\rangle|$. We have $x^{-1}\left(\Sigma^{\prime+}\right)=\left(\Sigma^{\prime+} \cap x^{-1}\left(\Sigma^{\prime+}\right)\right) \sqcup\left(\Sigma^{\prime-} \cap x^{-1}\left(\Sigma^{\prime+}\right)\right)=\left(\Sigma^{\prime+} \cap x^{-1}\left(\Sigma^{\prime+}\right)\right) \sqcup$ $\left(-\left(\Sigma^{\prime+} \cap x^{-1}\left(\Sigma^{\prime-}\right)\right)\right)$. Since

$$
\sum_{\alpha \in-\left(\Sigma^{\prime+} \cap x^{-1}\left(\Sigma^{\prime}\right)\right)}|\langle\alpha, \nu(\lambda)\rangle|=\sum_{\alpha \in\left(\Sigma^{\prime+} \cap x^{-1}\left(\Sigma^{\prime}\right)\right)}|\langle\alpha, \nu(\lambda)\rangle|
$$

we have

$$
\begin{aligned}
\ell(w \cdot \lambda) & =\sum_{\alpha \in\left(\Sigma^{\prime+} \cap x^{-1}\left(\Sigma^{\prime+}\right)\right)}|\langle\alpha, \nu(\lambda)\rangle|+\sum_{\alpha \in-\left(\Sigma^{\prime+} \cap x^{-1}\left(\Sigma^{\prime-}\right)\right)}|\langle\alpha, \nu(\lambda)\rangle| \\
& =\sum_{\alpha \in\left(\Sigma^{\prime+} \cap x^{-1}\left(\Sigma^{\prime+}\right)\right)}|\langle\alpha, \nu(\lambda)\rangle|+\sum_{\alpha \in\left(\Sigma^{\prime+} \cap x^{-1}\left(\Sigma^{\prime}\right)\right)}|\langle\alpha, \nu(\lambda)\rangle| \\
& =\sum_{\alpha \in \Sigma^{\prime+}}|\langle\alpha, \nu(\lambda)\rangle|=\ell(\lambda) .
\end{aligned}
$$

We finish the proof.

Lemma 2.16. Let $v \in W_{0}$ and $\lambda \in \Lambda(1)$. Then we have

$$
\begin{aligned}
\ell\left(\lambda n_{v}\right) & =\ell(\lambda)+\ell(v)-2 \#\left\{\alpha \in \Sigma_{\text {red }}^{+} \mid v^{-1}(\alpha)<0,\langle\alpha, \nu(\lambda)\rangle>0\right\} \\
& =\ell(\lambda)-\ell(v)+2 \#\left\{\alpha \in \Sigma_{\text {red }}^{+} \mid v^{-1}(\alpha)<0,\langle\alpha, \nu(\lambda)\rangle \leq 0\right\}
\end{aligned}
$$

and

$$
\begin{aligned}
\ell\left(n_{v} \lambda\right) & =\ell(\lambda)+\ell(v)-2 \#\left\{\alpha \in \Sigma_{\text {red }}^{+} \mid v(\alpha)<0,\langle\alpha, \nu(\lambda)\rangle<0\right\} \\
& =\ell(\lambda)-\ell(v)+2 \#\left\{\alpha \in \Sigma_{\text {red }}^{+} \mid v(\alpha)<0, \quad\langle\alpha, \nu(\lambda)\rangle \geq 0\right\} .
\end{aligned}
$$

Proof. The formula for $\ell\left(n_{v} \lambda\right)$ follows from the formula for $\ell\left(\lambda n_{v}\right)$ and $\ell\left(n_{v} \lambda\right)=\ell\left(\left(n_{v} \lambda\right)^{-1}\right)=\ell\left(\lambda^{-1} n_{v}^{-1}\right)=\ell\left(\lambda^{-1} n_{v^{-1}}\right)$, here, at the last point, we use the fact that $n_{v^{-1}} n_{v} \in \operatorname{Ker}(W(1) \rightarrow W)$ has the length zero.

Since

$$
|\langle\alpha, \nu(\lambda)\rangle-1|-1= \begin{cases}|\langle\alpha, \nu(\lambda)\rangle|-2 & (\langle\alpha, \nu(\lambda)\rangle>0) \\ |\langle\alpha, \nu(\lambda)\rangle| & (\langle\alpha, \nu(\lambda)\rangle \leq 0)\end{cases}
$$


we have

$$
\begin{aligned}
\ell\left(\lambda n_{v}\right)-\#\left(\Sigma^{\prime+} \cap v\left(\Sigma^{\prime-}\right)\right) \\
\quad=\sum_{\alpha \in \Sigma^{\prime+}}|\langle\alpha, \nu(\lambda)\rangle|-2 \#\left\{\alpha \in \Sigma^{\prime+} \cap v\left(\Sigma^{\prime-}\right) \mid\langle\alpha, \nu(\lambda)\rangle>0\right\} .
\end{aligned}
$$

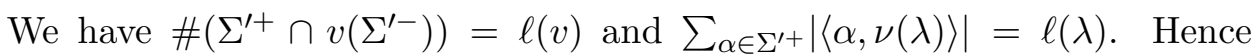
$\ell\left(\lambda n_{v}\right)=\ell(\lambda)+\ell(v)-2 \#\left\{\alpha \in \Sigma^{\prime+} \cap v\left(\Sigma^{\prime-}\right) \mid\langle\alpha, \nu(\lambda)\rangle>0\right\}$. For any $\alpha \in \Sigma^{\prime}$ there exists a unique $r_{\alpha}>0$ such that $r_{\alpha} \alpha \in \Sigma_{\text {red }}$. We have $\alpha \in \Sigma^{\prime+} \cap v\left(\Sigma^{\prime-}\right)$ if and only if $r_{\alpha} \alpha \in \Sigma_{\text {red }}^{+} \cap v\left(\Sigma_{\text {red }}^{-}\right)$and $\langle\alpha, \nu(\lambda)\rangle>0$ if and only if $\left\langle r_{\alpha} \alpha, \nu(\lambda)\right\rangle>0$. Since $\alpha \mapsto r_{\alpha} \alpha$ gives a bijection $\Sigma^{\prime} \simeq \Sigma_{\text {red }}$, we get $\#\left\{\alpha \in \Sigma^{\prime+} \cap v\left(\Sigma^{\prime-}\right) \mid\langle\alpha, \nu(\lambda)\rangle>0\right\}=\#\left\{\alpha \in \Sigma_{\text {red }}^{+} \cap v\left(\Sigma_{\text {red }}^{-}\right) \mid\langle\alpha, \nu(\lambda)\rangle>0\right\}$. We get the first formula. Since

$$
\begin{aligned}
\ell(v)= & \#\left\{\alpha \in \Sigma_{\text {red }}^{+} \mid v^{-1}(\alpha)<0\right\} \\
= & \#\left\{\alpha \in \Sigma_{\text {red }}^{+} \mid v^{-1}(\alpha)<0,\langle\alpha, \nu(\lambda)\rangle \leq 0\right\} \\
& +\#\left\{\alpha \in \Sigma_{\text {red }}^{+} \mid v^{-1}(\alpha)<0,\langle\alpha, \nu(\lambda)\rangle>0\right\},
\end{aligned}
$$

we get the second formula.

Lemma 2.17. Let $v \in W_{0}$ and $\lambda \in \Lambda(1)$. We have:

- $\ell\left(\lambda n_{v}\right)=\ell(\lambda)+\ell(v)$ if and only if for any $\alpha \in \Sigma^{+}$such that $v^{-1}(\alpha)<$ $0,\langle\alpha, \nu(\lambda)\rangle \leq 0$.

- $\ell\left(\lambda n_{v}\right)=\ell(\lambda)-\ell(v)$ if and only if for any $\alpha \in \Sigma^{+}$such that $v^{-1}(\alpha)<$ $0,\langle\alpha, \nu(\lambda)\rangle>0$.

- $\ell\left(n_{v} \lambda\right)=\ell(\lambda)+\ell(v)$ if and only if for any $\alpha \in \Sigma^{+}$such that $v(\alpha)<0$, $\langle\alpha, \nu(\lambda)\rangle \geq 0$.

- $\ell\left(n_{v} \lambda\right)=\ell(\lambda)-\ell(v)$ if and only if for any $\alpha \in \Sigma^{+}$such that $v(\alpha)<0$, $\langle\alpha, \nu(\lambda)\rangle<0$.

In particular, for $\alpha \in \Delta$ and $\lambda \in \Lambda(1)$, we have

- $\ell\left(\lambda n_{s_{\alpha}}\right)=\ell(\lambda)+1$ if and only if $\langle\alpha, \nu(\lambda)\rangle \leq 0$.

- $\ell\left(n_{s_{\alpha}} \lambda\right)=\ell(\lambda)+1$ if and only if $\langle\alpha, \nu(\lambda)\rangle \geq 0$.

Proof. Obvious from the previous lemma.

Lemma 2.18. Let $P$ be a parabolic subgroup, $v \in W_{0}, w \in W_{P}(1)$ and $\lambda_{0} \in Z\left(W_{P}(1)\right)$.

(1) If $v \in W_{0}^{P}, \lambda_{0}$ is dominant and $w$ is $P$-negative, then we have $\ell\left(n_{v} \lambda_{0} w\right)=\ell\left(n_{v} \lambda_{0}\right)+\ell(w)=\ell(v)+\ell\left(\lambda_{0}\right)+\ell(w)$.

(2) If $v \in{ }^{P} W_{0}, \lambda_{0}$ is anti-dominant and $w$ is $P$-positive, then we have $\ell\left(w \lambda_{0} n_{v}\right)=\ell(w)+\ell\left(\lambda_{0} n_{v}\right)=\ell(w)+\ell\left(\lambda_{0}\right)+\ell(v)$.

Proof. (2) follows from (11) by taking the inverse.

We prove (1). Take $w_{1} \in W_{0, P}$ and $\lambda \in \Lambda(1)$ such that $w=n_{w_{1}} \lambda$. We remark that $\nu\left(\lambda_{0}\right)$ and $\nu(\lambda)$ is in the same closed Weyl chamber. In fact, if $\alpha \in \Sigma^{+} \backslash \Sigma_{P}^{+}$, then $\left\langle\alpha, \nu\left(\lambda_{0}\right)\right\rangle \geq 0$ and $\langle\alpha, \nu(\lambda)\rangle \geq 0$ by the assumption. Hence $\left\langle\alpha, \nu\left(\lambda_{0}\right)\right\rangle\langle\alpha, \nu(\lambda)\rangle \geq 0$. If $\alpha \in \Sigma_{P}^{+}$, then $\left\langle\alpha, \nu\left(\lambda_{0}\right)\right\rangle=0$ since $\lambda_{0} \in$ $Z\left(W_{P}(1)\right)$. Hence $\langle\alpha, \nu(\lambda)\rangle\left\langle\alpha, \nu\left(\lambda_{0}\right)\right\rangle \geq 0$ for any $\alpha \in \Sigma_{P}^{+}$. Therefore $\nu(\lambda)$ and $\nu\left(\lambda_{0}\right)$ are in the same closed Weyl chamber. In particular, $\ell\left(\lambda_{0} \lambda\right)=$ $\ell\left(\lambda_{0}\right)+\ell(\lambda)$ by Lemma 2.11. 
Since $\lambda_{0}$ is in the center of $W_{P}(1)$, we have

$$
\begin{aligned}
\ell\left(n_{v} \lambda_{0} w\right) & =\ell\left(n_{v} n_{w_{1}} \lambda_{0} \lambda\right) \\
& =\ell\left(\lambda_{0} \lambda\right)+\ell\left(v w_{1}\right)-2 \#\left\{\alpha \in \Sigma_{\text {red }}^{+} \mid v w_{1}(\alpha)<0,\left\langle\alpha, \nu\left(\lambda_{0} \lambda\right)\right\rangle<0\right\}
\end{aligned}
$$

by Lemma 2.16 .

Let $\alpha \in \Sigma_{\text {red }}^{+}$such that $v w_{1}(\alpha)<0$. Since $v \in W_{0}^{P}, \ell\left(v w_{1}\right)=\ell(v)+\ell\left(w_{1}\right)$. Hence we have $w_{1}(\alpha)<0$ or $v(\beta)<0$ where $\beta=w_{1}(\alpha)>0$. Assume that $v(\beta)<0$ where $\beta=w_{1}(\alpha)>0$. Since $v \in W_{0}^{P}, \beta \in \Sigma^{+} \backslash \Sigma_{P}^{+}$. Therefore $\alpha=w_{1}^{-1}(\beta) \in \Sigma^{+} \backslash \Sigma_{P}^{+}$. Hence $\langle\alpha, \nu(\lambda)\rangle \geq 0$ since $\lambda$ is $P$-negative. From the assumption, $\lambda_{0}$ is dominant. Therefore $\left\langle\alpha, \nu\left(\lambda_{0}\right)\right\rangle \geq 0$. Hence we get $\left\langle\alpha, \nu\left(\lambda_{0} \lambda\right)\right\rangle \geq 0$. Therefore we have

$\ell\left(n_{v} \lambda_{0} w\right)=\ell\left(\lambda_{0} \lambda\right)+\ell\left(v w_{1}\right)-2 \#\left\{\alpha \in \Sigma_{\text {red }}^{+} \mid w_{1}(\alpha)<0,\left\langle\alpha, \nu\left(\lambda_{0} \lambda\right)\right\rangle<0\right\}$.

Since $w_{1} \in W_{0, P}, w_{1}(\alpha)<0$ implies $\alpha \in \Sigma_{P}^{+}$. Hence $\left\langle\alpha, \nu\left(\lambda_{0}\right)\right\rangle=0$. We have

$$
\ell\left(n_{v} \lambda_{0} w\right)=\ell\left(\lambda_{0} \lambda\right)+\ell\left(v w_{1}\right)-2 \#\left\{\alpha \in \Sigma_{\text {red }}^{+} \mid w_{1}(\alpha)<0,\langle\alpha, \nu(\lambda)\rangle<0\right\} .
$$

Recall that we have $\ell\left(v w_{1}\right)=\ell(v)+\ell\left(w_{1}\right)$ and $\ell\left(\lambda_{0} \lambda\right)=\ell\left(\lambda_{0}\right)+\ell(\lambda)$. Hence

$$
\begin{aligned}
& \ell\left(n_{v} \lambda_{0} w\right) \\
& =\ell\left(\lambda_{0}\right)+\ell(v)+\ell(\lambda)+\ell\left(w_{1}\right)-2 \#\left\{\alpha \in \Sigma_{\text {red }}^{+} \mid w_{1}(\alpha)<0,\langle\alpha, \nu(\lambda)\rangle<0\right\} \\
& =\ell\left(\lambda_{0}\right)+\ell(v)+\ell\left(n_{w_{1}} \lambda\right)=\ell\left(\lambda_{0}\right)+\ell(v)+\ell(w)
\end{aligned}
$$

by Lemma 2.16. Put $w=1$. Then we have $\ell\left(n_{v} \lambda_{0}\right)=\ell\left(\lambda_{0}\right)+\ell(v)$.

Lemma 2.19. Let $w \in W_{P}(1), v \in W_{0}$ and $\lambda_{0} \in Z\left(W_{P}(1)\right)$.

(1) If $w$ is P-positive, $\lambda_{0}=\lambda_{P}^{+}$as in Proposition 2.5 and $v \in W_{0}^{P}$, then $\ell\left(n_{v} \lambda_{0} w\right)=\ell\left(n_{v} \lambda_{0}\right)+\ell(w)=\ell\left(\lambda_{0}\right)-\ell(v)+\ell(w)$.

(2) If $w$ is $P$-negative, $\lambda_{0}=\lambda_{P}^{-}$as in Proposition 2.5 and $v \in{ }^{P} W_{0}$, then $\ell\left(w \lambda_{0} n_{v}\right)=\ell(w)+\ell\left(\lambda_{0} n_{v}\right)=\ell(w)+\ell\left(\lambda_{0}\right)-\ell(v)$.

Proof. (2) follows from (11) by taking the inverse.

Take $w_{1} \in W_{0, P}$ and $\lambda \in \Lambda(1)$ such that $w=n_{w_{1}} \lambda$. Then we have

$$
\begin{aligned}
& \ell\left(n_{v} \lambda_{0} w\right) \\
& =\ell\left(n_{v} n_{w_{1}} \lambda_{0} \lambda\right) \\
& =\ell\left(\lambda_{0} \lambda\right)-\ell\left(v w_{1}\right)+2 \#\left\{\alpha \in \Sigma_{\text {red }}^{+} \mid\left(v w_{1}\right)(\alpha)<0,\left\langle\alpha, \nu\left(\lambda_{0} \lambda\right)\right\rangle \geq 0\right\}
\end{aligned}
$$

by Lemma 2.16. Let $\alpha \in \Sigma^{+}$such that $\left(v w_{1}\right)(\alpha)<0$. Then $w_{1}(\alpha)<0$ or $v(\beta)<0$ where $\beta=w_{1}(\alpha)>0$. If $v(\beta)<0, \beta=w_{1}(\alpha)>0$, then $\beta \in \Sigma^{+} \backslash \Sigma_{P}^{+}$since $v \in W_{0}^{P}$. Since $w_{1} \in W_{0, P}$, we have $\alpha=w_{1}^{-1}(\beta) \in \Sigma^{+} \backslash \Sigma_{P}^{+}$. Hence $\left\langle\alpha, \nu\left(\lambda_{0}\right)\right\rangle<0$ by the condition on $\lambda_{P}^{+},\langle\alpha, \nu(\lambda)\rangle \leq 0$ since $\lambda$ is $P$ positive. Therefore we have $\left\langle\alpha, \nu\left(\lambda_{0} \lambda\right)\right\rangle<0$. We get

$\ell\left(n_{v} \lambda_{0} w\right)=\ell\left(\lambda_{0} \lambda\right)-\ell\left(v w_{1}\right)+2 \#\left\{\alpha \in \Sigma_{\text {red }}^{+} \mid w_{1}(\alpha)<0,\left\langle\alpha, \nu\left(\lambda_{0} \lambda\right)\right\rangle \geq 0\right\}$. If $w_{1}(\alpha)<0$, then since $w_{1} \in W_{0, P}$, we have $\alpha \in \Sigma_{P}$. Hence $\left\langle\alpha, \nu\left(\lambda_{0}\right)\right\rangle=0$. Therefore we have

$$
\ell\left(n_{v} \lambda_{0} w\right)=\ell\left(\lambda_{0} \lambda\right)-\ell\left(v w_{1}\right)+2 \#\left\{\alpha \in \Sigma_{\text {red }}^{+} \mid w_{1}(\alpha)<0,\langle\alpha, \nu(\lambda)\rangle \geq 0\right\} .
$$

We have:

- $\ell\left(v w_{1}\right)=\ell(v)+\ell\left(w_{1}\right)$ since $v \in W_{0}^{P}$ and $w_{1} \in W_{0, P}$. 
- $\ell\left(\lambda_{0} \lambda\right)=\ell\left(\lambda_{0}\right)+\ell(\lambda)$. Indeed, $\nu\left(\lambda_{0}\right)$ and $\nu(\lambda)$ are in the same closed Weyl chamber. If $\alpha \in \Sigma^{+} \backslash \Sigma_{P}^{+}$, then $\left\langle\alpha, \nu\left(\lambda_{0}\right)\right\rangle$ and $\langle\alpha, \nu(\lambda)\rangle$ are both not positive since $\lambda_{0}, \lambda$ are both $P$-positive. If $\alpha \in \Sigma_{P}^{+}$, then $\left\langle\alpha, \nu\left(\lambda_{0}\right)\right\rangle=0$, hence $\left\langle\alpha, \nu\left(\lambda_{0}\right)\right\rangle\langle\alpha, \nu(\lambda)\rangle=0 \geq 0$.

Therefore we get

$$
\begin{aligned}
& \ell\left(n_{v} \lambda_{0} w\right) \\
& =\ell\left(\lambda_{0}\right)-\ell(v)+\ell(\lambda)-\ell\left(w_{1}\right)+2 \#\left\{\alpha \in \Sigma_{\text {red }}^{+} \mid w_{1}(\alpha)<0,\langle\alpha, \nu(\lambda)\rangle \geq 0\right\} \\
& =\ell\left(\lambda_{0}\right)-\ell(v)+\ell\left(\lambda n_{w_{1}}\right)=\ell\left(\lambda_{0}\right)-\ell(v)+\ell(w)
\end{aligned}
$$

by Lemma 2.16, Applying this to $w=1$, we get $\ell\left(n_{v} \lambda_{0}\right)=\ell\left(\lambda_{0}\right)-\ell(v)$.

Lemma 2.20. Let $w \in W_{P}(1)$ and $\lambda_{P}^{-} \in \Lambda(1)$ as in Proposition 2.5. Then $\ell\left(w \lambda_{P}^{-}\right)=\ell(w)+\ell\left(\lambda_{P}^{-}\right)$if and only if $w$ is $P$-negative.

Proof. The "if part" follows from Lemma 2.19 (2). Assume that $\ell\left(w \lambda_{P}^{-}\right)=$ $\ell(w)+\ell\left(\lambda_{P}^{-}\right)$. Take $v \in W_{0, P}$ and $\mu \in \Lambda(1)$ such that $w=n_{v} \mu$. Then we have

$$
\ell\left(w \lambda_{P}^{-}\right)=\sum_{\alpha \in \Sigma^{\prime+} \cap v^{-1}\left(\Sigma^{\prime+}\right)}\left|\left\langle\alpha, \nu\left(\mu \lambda_{P}^{-}\right)\right\rangle\right|+\sum_{\alpha \in \Sigma^{\prime+} \cap v^{-1}\left(\Sigma^{\prime-}\right)}\left|\left\langle\alpha, \nu\left(\mu \lambda_{P}^{-}\right)\right\rangle+1\right|
$$

and

$$
\begin{aligned}
\ell(w) & =\sum_{\alpha \in \Sigma^{\prime+} \cap v^{-1}\left(\Sigma^{\prime+}\right)}|\langle\alpha, \nu(\mu)\rangle|+\sum_{\alpha \in \Sigma^{\prime+} \cap v^{-1}\left(\Sigma^{\prime}\right)}|\langle\alpha, \nu(\mu)\rangle+1|, \\
\ell\left(\lambda_{P}^{-}\right) & =\sum_{\alpha \in \Sigma^{\prime+} \cap v^{-1}\left(\Sigma^{\prime+}\right)}\left|\left\langle\alpha, \nu\left(\lambda_{P}^{-}\right)\right\rangle\right|+\sum_{\alpha \in \Sigma^{\prime+} \cap v^{-1}\left(\Sigma^{\prime}\right)}\left|\left\langle\alpha, \nu\left(\lambda_{P}^{-}\right)\right\rangle\right|
\end{aligned}
$$

by the length formula. By the triangle inequality and the assumption $\ell\left(w \lambda_{P}^{-}\right)=\ell(w)+\ell\left(\lambda_{P}^{-}\right)$, we have

$$
\left|\left\langle\alpha, \nu\left(\mu \lambda_{P}^{-}\right)\right\rangle+\varepsilon\right|=|\langle\alpha, \nu(\mu)\rangle+\varepsilon|+\left|\left\langle\alpha, \nu\left(\lambda_{P}^{-}\right)\right\rangle\right| .
$$

where $\varepsilon=1$ if $\alpha \in \Sigma^{\prime+} \cap v^{-1}\left(\Sigma^{\prime-}\right)$ and $\varepsilon=0$ if $\alpha \in \Sigma^{\prime+} \cap v^{-1}\left(\Sigma^{\prime+}\right)$. If $\alpha \in \Sigma^{+} \backslash \Sigma_{P}^{+}$, we have $v(\alpha)>0$ since $v \in W_{0, P}$. Hence $\varepsilon=0$. Therefore we get

$$
\left|\left\langle\alpha, \nu\left(\mu \lambda_{P}^{-}\right)\right\rangle\right|=|\langle\alpha, \nu(\mu)\rangle|+\left|\left\langle\alpha, \nu\left(\lambda_{P}^{-}\right)\right\rangle\right|,
$$

So we get $\langle\alpha, \nu(\mu)\rangle\left\langle\alpha, \nu\left(\lambda_{P}^{-}\right)\right\rangle \geq 0$. We have $\left\langle\alpha, \nu\left(\lambda_{P}^{-}\right)\right\rangle>0$ by the condition on $\lambda_{P}^{-}$. Hence $\langle\alpha, \nu(\mu)\rangle \geq 0$. Therefore $w=n_{v} \mu$ is $P$-negative.

2.11. Twist by $n_{w_{G} w_{P}}$. For a parabolic subgroup $P$, let $w_{P}$ be the longest element in $W_{0, P}$. In particular, $w_{G}$ is the longest element in $W_{0}$. Let $P^{\prime}$ be a parabolic subgroup corresponding to $-w_{G}\left(\Delta_{P}\right)$, in other words, $P^{\prime}=n_{w_{G} w_{P}} P^{\mathrm{op}} n_{w_{G} w_{P}}^{-1}$ where $P^{\mathrm{op}}$ is the opposite parabolic subgroup of $P$ with respect to the Levi part of $P$ containing $Z$. Set $n=n_{w_{G} w_{P}}$. Then the map $P^{\mathrm{op}} \rightarrow P^{\prime}$ defined by $p \mapsto n p n^{-1}$ is an isomorphism which preserves the data used to define the pro- $p$-Iwahori Hecke algebras. Hence $T_{w}^{P} \mapsto T_{n w n^{-1}}^{P^{\prime}}$ gives an isomorphism $\mathcal{H}_{P} \rightarrow \mathcal{H}_{P^{\prime}}$. This sends $T_{w}^{P *}$ to $T_{n w n^{-1}}^{P^{\prime *}}$ and $E_{o_{+, P} \cdot v}^{P}(w)$ to $E_{o_{+, P^{\prime}} \cdot n v n^{-1}}^{P^{\prime}}\left(n w n^{-1}\right)$ where $v \in W_{0, P}$. 
Let $\sigma$ be an $\mathcal{H}_{P}$-module. Then we define an $\mathcal{H}_{P^{\prime}}$-module $n_{w_{G} w_{P}} \sigma$ via the pull-back of the above isomorphism. Namely, we define $\left(n_{w_{G} w_{P}} \sigma\right)\left(T_{w}^{P^{\prime}}\right)=$ $\sigma\left(T_{n_{w_{G} w_{P}}^{-1} w n_{w_{G} w_{P}}^{P}}^{P}\right)$.

Using this twist, we have another description of $I_{P}$.

Proposition 2.21 ([Vig15b, Theorem 1.8]). Let $P$ be a parabolic subgroup and $\sigma$ an $\mathcal{H}_{P}$-module. Set $P^{\prime}=n_{w_{G} w_{P}} P^{\mathrm{op}} n_{w_{G} w_{P}}^{-1}$. Then the map $\varphi \mapsto$ $\varphi\left(T_{n_{w}}\right)$ gives an isomorphism

$$
\left.\left\{\varphi \in I_{P}(\sigma) \mid \varphi\left(T_{n_{w}}\right)=0 \text { for any } w \in W_{0}^{P} \backslash\left\{w_{G} w_{P}\right\}\right\} \simeq n_{w_{G} w_{P}} \sigma\right|_{\mathcal{H}_{P^{\prime}}^{+}}
$$

as $j_{P^{\prime}}^{+}\left(\mathcal{H}_{P^{\prime}}^{+}\right)$-modules. Let $x \mapsto \varphi_{x}$ be the inverse of the above isomorphism. Then the induced homomorphism

$$
n_{w_{G} w_{P}} \sigma \otimes_{\left(\mathcal{H}_{P^{\prime}}^{+}, j_{P^{\prime}}^{+}\right)} \mathcal{H} \rightarrow I_{P}(\sigma)
$$

given by $x \otimes X \mapsto \varphi_{x} X$ is an isomorphism.

Lemma 2.22. (1) The map $w \mapsto w_{G} w_{P} w^{-1}$ gives a bijection $W_{0}^{P} \simeq$ ${ }^{\prime} W_{0}$ which reverse the Bruhat order.

(2) The isomorphism $I_{P}(\sigma) \simeq n_{w_{G} w_{P}} \sigma \otimes_{\left(\mathcal{H}_{P^{\prime}}^{+}, j_{P^{\prime}}^{+}\right)} \mathcal{H}$ is given by $I_{P}(\sigma) \ni$ $\varphi \mapsto \sum_{w \in W_{0}^{P}} \varphi\left(T_{n_{w}}\right) \otimes T_{n_{w_{G} w_{P} w^{-1}}^{*}}^{*}$.

Proof. The first part is proved in the proof of [Abe, Proposition 4.15]. For (2), we prove the following. Let $x \in \sigma$ and $\varphi \in I_{P}(\sigma)$ such that $\varphi\left(T_{n_{w_{G} w_{P}}}\right)=$ $x$ and $\varphi\left(T_{n_{w}}\right)=0$ for any $w \in W_{0}^{P} \backslash\left\{w_{G} w_{P}\right\}$. Then for $v \in{ }^{P} W_{0}$ and $w \in W_{0}^{P}$, we have

$$
\left(\varphi T_{n_{v}}^{*}\right)\left(T_{n_{w}}\right)= \begin{cases}x & v=w_{G} w_{P} w^{-1} \\ 0 & \text { otherwise. }\end{cases}
$$

This means the following diagram is commutative.

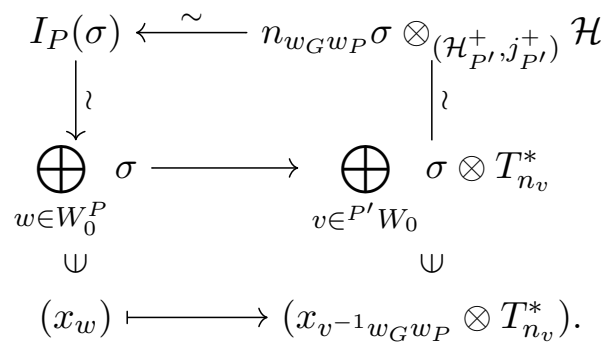

The commutativity of this diagram implies the lemma.

Assume that $\left(\varphi T_{n_{v}}^{*}\right)\left(T_{n_{w}}\right) \neq 0$. We have

$$
T_{n_{v}}^{*} T_{n_{w}}=E_{v^{-1} \cdot o_{-}}\left(n_{v}\right) E_{o_{-}}\left(n_{w}\right) \in C E_{v^{-1} \cdot o_{-}}\left(n_{v} n_{w}\right) \subset \sum_{a \leq v w} T_{n_{a}} C\left[Z_{\kappa}\right] .
$$

For $a \in W_{0}$ and $t \in Z_{\kappa}$, we have $\varphi\left(T_{n_{a}} T_{t}\right)=\varphi\left(T_{n_{a}}\right) T_{t}^{P}$. Hence $\varphi\left(T_{n_{a}}\right) \neq 0$ for some $a \leq v w$.

Decompose $a=a_{1} a_{2}$ where $a_{1} \in W_{0}^{P}$ and $a_{2} \in W_{0, P}$. Then we have $\varphi\left(T_{n_{a}}\right)=\varphi\left(T_{n_{a_{1}}}\right) T_{n_{a_{2}}}^{P}$. Since this is non-zero, we have $a_{1}=w_{G} w_{P}$. Namely we have $a \in w_{G} W_{0, P}$. Take $b \in W_{0, P}$ such that $a=w_{G} b$. By (1), we can take $v_{1} \in W_{0}^{P}$ such that $v=w_{G} w_{P} v_{1}^{-1}$. Then $a \leq v w$ implies $b \geq w_{P} v_{1}^{-1} w$. Since 
$b \in W_{0, P}$, we also have $w_{P} v_{1}^{-1} w \in W_{0, P}$. Hence $v_{1}^{-1} w \in W_{0, P}$. Therefore we have $w \in v_{1} W_{0, P}$. Since $v_{1}, w \in W_{0}^{P}$, we have $v_{1}=w$. Hence $v=w_{G} w_{P} w^{-1}$.

If $v=w_{G} w_{P} w^{-1}$, then $\ell(v)=\ell\left(w_{G}\right)-\ell\left(w_{P} w^{-1}\right)=\ell\left(w_{G}\right)-\ell\left(w_{P}\right)-\ell(w)$ since $w \in W_{0}^{P}$. Hence $\ell(v)+\ell(w)=\ell\left(w_{G}\right)-\ell\left(w_{P}\right)=\ell\left(w_{G} w_{P}\right)=\ell(v w)$. Therefore we have

$$
\begin{aligned}
T_{n_{v}}^{*} T_{n_{w}} & =E_{o_{-} \cdot v^{-1}}\left(n_{v}\right) E_{o_{-}}\left(n_{w}\right) \\
& =E_{o_{-} \cdot v^{-1}}\left(n_{v} n_{w}\right) \in T_{n_{w_{G} w_{P}}}+\sum_{a<w_{G} w_{P}} T_{n_{a}} C\left[Z_{\kappa}\right] .
\end{aligned}
$$

By [Abe, Lemma 4.13], if $a<w_{G} w_{P}$ then $a \notin w_{G} w_{P} W_{0, P}$. Hence we have $\varphi\left(T_{n_{a}} C\left[Z_{\kappa}\right]\right)=0$. Therefore we have $\varphi\left(T_{n_{v}}^{*} T_{n_{w}}\right)=\varphi\left(T_{n_{w_{G} w_{P}}}\right)=x$.

2.12. The extension and the generalized Steinberg modiles. Let $P$ be a parabolic subgroup and $\sigma$ an $\mathcal{H}_{P}$-module. For $\alpha \in \Delta$, let $P_{\alpha}$ be a parabolic subgroup corresponding to $\{\alpha\}$. Then we define $\Delta(\sigma) \subset \Delta$ by

$\Delta(\sigma)$

$=\left\{\alpha \in \Delta \mid\left\langle\Delta_{P}, \alpha^{\vee}\right\rangle=0, \sigma\left(T_{\lambda}^{P}\right)=1\right.$ for any $\left.\lambda \in W_{\text {aff }, P_{\alpha}}(1) \cap \Lambda(1)\right\} \cup \Delta_{P}$.

Let $P(\sigma)$ be a parabolic subgroup corresponding to $\Delta(\sigma)$.

Proposition 2.23 (AHVa]). Let $\sigma$ be an $\mathcal{H}_{P}$-module and $Q$ a parabolic subgroup between $P$ and $P(\sigma)$. Denote the parabolic subgroup corresponding to $\Delta_{Q} \backslash \Delta_{P}$ by $P_{2}$. Then there exist a unique $\mathcal{H}_{Q}$-module $e_{Q}(\sigma)$ acting on the same space as $\sigma$ such that

- $e_{Q}(\sigma)\left(T_{w}^{Q *}\right)=\sigma\left(T_{w}^{P *}\right)$ for any $w \in W_{P}(1)$.

- $e_{Q}(\sigma)\left(T_{w}^{Q *}\right)=1$ for any $w \in W_{P_{2}, \text { aff }}(1)$.

Moreover, one of the following condition gives a characterization of $e_{Q}(\sigma)$.

(1) For any $w \in W_{P}^{Q-}(1), e_{Q}(\sigma)\left(T_{w}^{Q *}\right)=\sigma\left(T_{w}^{P *}\right)$ (namely, $e_{Q}(\sigma) \simeq \sigma$ as $\left(\mathcal{H}_{P}^{Q-}, j_{P}^{Q-*}\right)$-modules) and for any $w \in W_{\mathrm{aff}, P_{2}}(1), e_{Q}(\sigma)\left(T_{w}^{Q *}\right)=1$.

(2) For any $w \in W_{P}^{Q+}(1), e_{Q}(\sigma)\left(T_{w}^{Q *}\right)=\sigma\left(T_{w}^{P *}\right)$ and for any $w \in$ $W_{\text {aff }, P_{2}}(1), e_{Q}(\sigma)\left(T_{w}^{Q *}\right)=1$.

We call $e_{Q}(\sigma)$ the extension of $\sigma$ to $\mathcal{H}_{Q}$. A typical example of the extension is the trivial representation $\mathbf{1}=\mathbf{1}_{G}$. This is a one-dimensional $\mathcal{H}$-module defined by $\mathbf{1}\left(T_{w}\right)=q_{w}$, or equivalently $\mathbf{1}\left(T_{w}^{*}\right)=1$. We have $\Delta\left(\mathbf{1}_{P}\right)=\left\{\alpha \in \Delta \mid\left\langle\Delta_{P}, \alpha^{\vee}\right\rangle=0\right\} \cup \Delta_{P}$ and, if $Q$ is a parabolic subgroup between $P$ and $P\left(\mathbf{1}_{P}\right)$, we have $e_{Q}\left(\mathbf{1}_{P}\right)=\mathbf{1}_{Q}$

Remark 2.24. Assume that $p=0$ in $C$. The condition "for any $w \in$ $W_{\text {aff, } P_{2}}(1), e_{Q}(\sigma)\left(T_{w}^{Q *}\right)=1$ " is equivalent to the following two conditions.

- $e_{Q}(\sigma)\left(T_{s}^{Q}\right)=0$ for any $s \in S_{\text {aff, } P_{2}}(1) \cap W_{\text {aff }, P_{2}}(1)$.

- $e_{Q}(\sigma)\left(T_{t}^{Q}\right)=1$ for any $t \in Z_{\kappa} \cap W_{\text {aff }, P_{2}}(1)$.

Indeed, assume that $e_{Q}(\sigma)\left(T_{w}^{Q *}\right)=1$ for any $w \in W_{\text {aff, } P_{2}}(1)$. Then for $t \in Z_{\kappa} \cap W_{\text {aff, } P_{2}}(1)$, we have $T_{t}^{Q *}=T_{t}^{Q}$. Hence $e_{Q}(\sigma)\left(T_{t}^{Q}\right)=e_{Q}(\sigma)\left(T_{t}^{Q *}\right)=$ 1. For $s \in S_{\text {aff, } P_{2}}(1) \cap W_{\text {aff, } P_{2}}(1)$, take $c_{s}(t) \in \mathbb{Z}$ as in Proposition 2.1, By Lemma 2.3, we have $e_{Q}(\sigma)\left(c_{s}\right)=\sum_{t \in Z_{\kappa} \cap W_{\text {aff, } P_{2}}(1)} c_{s}(t) e_{Q}(\sigma)\left(T_{t}^{Q}\right)=$ $\sum_{t \in Z_{\kappa} \cap W_{\text {aff }, P_{2}}(1)} c_{s}(t)=q_{s, P_{2}}-1=-1$. Hence $e_{Q}(\sigma)\left(T_{s}^{Q}\right)=e_{Q}(\sigma)\left(T_{s}^{Q *}\right)+$ $e_{Q}(\sigma)\left(c_{s}\right)=1-1=0$. 
On the other hand, assume that the two conditions hold. Then by the above argument, from the second condition, we have $e_{Q}(\sigma)\left(c_{s}\right)=-1$. Hence $e_{Q}(\sigma)\left(T_{s}^{Q *}\right)=e_{Q}(\sigma)\left(T_{s}^{Q}\right)-e_{Q}(\sigma)\left(c_{s}\right)=1$. By taking a reduced expression of $w \in W_{\text {aff, } P_{2}}(1)$, we get $e_{Q}(\sigma)\left(T_{w}^{Q *}\right)=1$. The conditions are appeared in [Abe, 4.4].

Remark 2.25. For each $\alpha \in \Delta$, let $P_{\alpha}$ be a parabolic subgroup corresponding to $\{\alpha\}$. By [Abe, Lemma 2.5], $\Lambda(1) \cap W_{\text {aff, } P_{2}}(1)$ is generated by $\bigcup_{\alpha \in \Delta(\sigma) \backslash \Delta_{P}}\left(\Lambda(1) \cap W_{\text {aff, } P_{\alpha}}(1)\right)$. Hence for each $\lambda \in \Lambda(1) \cap W_{\text {aff, } P_{2}}(1)$, we can write $\lambda=\mu_{1} \cdots \mu_{r}$ where $\mu_{i} \in W_{\text {aff, } P_{\alpha}}(1) \cap \Lambda(1)$ for some $\alpha \in \Delta(\sigma) \backslash \Delta_{P}$. Since $\alpha \in \Delta(\sigma) \backslash \Delta_{P}$ is orthogonal to $\Delta_{P}, \ell_{P}\left(\mu_{i}\right)=0$ for each $i$. Therefore $T_{\lambda}^{P}=T_{\mu_{1}}^{P} \cdots T_{\mu_{r}}^{P}$. Since $\sigma\left(T_{\mu_{i}}^{P}\right)=1$, we have $\sigma\left(T_{\lambda}^{P}\right)=1$.

Let $P(\sigma) \supset P_{0} \supset Q_{1} \supset Q \supset P$. Then as in [Abe, 4.5], we have $I_{Q_{1}}^{P_{0}}\left(e_{Q_{1}}(\sigma)\right) \subset I_{Q}^{P_{0}}\left(e_{Q}(\sigma)\right)$. Define

$$
\operatorname{St}_{Q}^{P_{0}}(\sigma)=\operatorname{Cok}\left(\bigoplus_{Q_{1} \supsetneq Q} I_{Q_{1}}^{P_{0}}\left(e_{Q_{1}}(\sigma)\right) \rightarrow I_{Q}^{P_{0}}\left(e_{Q}(\sigma)\right)\right) .
$$

When $P_{0}=G$, we write $\operatorname{St}_{Q}(\sigma)$.

In the rest of this subsection, we assume that $P(\sigma)=G$. Since $\Delta \backslash \Delta_{P}=$ $\Delta(\sigma) \backslash \Delta_{P}$ is orthogonal to $\Delta_{P}$, we have $w_{G} w_{P} \in W_{0, P_{2}}$ where $P_{2}$ corresponds to $\Delta \backslash \Delta_{P}$ and $n_{w_{G} w_{P}} P^{\mathrm{op}} n_{w_{G} w_{P}}^{-1}=P$. Hence $n_{w_{G} w_{P}} \sigma$ is also an $\mathcal{H}_{P}$-module.

Lemma 2.26. $n_{w_{G} w_{P}} \sigma=\sigma$.

Proof. Let $w \in W_{P}(1)$. Put $n=n_{w_{G} w_{P}}$. Since $n \in W_{\text {aff, } P_{2}}(1)$ and $W_{\text {aff, } P_{2}}(1)$ is a normal subgroup of $W(1)$ [Abe, Lemma 4.17], we have $n^{-1} w n w^{-1}=$ $n^{-1}\left(w n w^{-1}\right) \in W_{\text {aff }, P_{2}}(1)$. The image of $n$ (resp. $w$ ) by $W(1) \rightarrow W \rightarrow W_{0}$ is in $W_{0, P_{2}}$ (resp. $W_{0, P}$ ) and by the assumption $P(\sigma)=G, W_{0, P_{2}}$ and $W_{0, P}$ commute with each other. Hence the image of $n^{-1} w n w^{-1}$ in $W_{0}$ is trivial. Therefore $n^{-1} w n w^{-1} \in \Lambda(1) \cap W_{\text {aff, } P_{2}}(1)$. In particular, the length as an element in $W_{P}(1)$ is zero by Lemma 2.12 and $\sigma\left(T_{n^{-1} w n w^{-1}}^{P}\right)=$ 1 by Remark 2.25. Hence $n \sigma\left(T_{w}^{P}\right)=\sigma\left(T_{n^{-1} w n}^{P}\right)=\sigma\left(T_{n^{-1} w n w^{-1}}^{P} T_{w}^{P}\right)=$ $\sigma\left(T_{n^{-1} w n w^{-1}}^{P}\right) \sigma\left(T_{w}^{P}\right)=\sigma\left(T_{w}^{P}\right)$.

Lemma 2.27. Let $Q$ be a parabolic subgroup containing $P$ and set $Q^{\prime}=$ $n_{w_{G} w_{Q}} Q^{\mathrm{op}} n_{w_{G} w_{Q}}^{-1}$. Then we have $n_{w_{G} w_{Q}} e_{Q}(\sigma) \simeq e_{Q^{\prime}}(\sigma)$.

Proof. By the above lemma, we have

$$
\left.\sigma\right|_{\mathcal{H}_{P}^{-}}=\left.n_{w_{G} w_{P}} \sigma\right|_{\mathcal{H}_{P}^{-}}=\left.n_{w_{G} w_{Q}} e_{Q}\left(n_{w_{Q} w_{P}} \sigma\right)\right|_{\mathcal{H}_{P}^{-}}=\left.n_{w_{G} w_{Q}} e_{Q}(\sigma)\right|_{\mathcal{H}_{P}^{-}} .
$$

Let $Q_{2}$ (resp. $Q_{2}^{\prime}$ ) be the subgroup corresponding to $\Delta_{Q} \backslash \Delta_{P}$ (resp. $\left.\Delta_{Q^{\prime}} \backslash \Delta_{P}\right)$. We have $w_{G} w_{Q} \in W_{0, P_{2}}$. Hence $n_{w_{G} w_{Q}}$ preserves $\Sigma_{P_{2}}$. Moreover we have $w_{G} w_{Q}\left(\Sigma_{Q_{2}}\right)=\Sigma_{Q_{2}^{\prime}}$. For $\alpha \in \Sigma_{Q_{2}}$, the root subgroup for $\alpha$ is sent to that of $w_{G} w_{Q}(\alpha)$ by $n_{w_{G} w_{Q}}$. Denote the Levi part of $Q_{2}$ (resp. $Q_{2}^{\prime}$ ) containing $Z$ by $M_{Q_{2}}$ (resp. $M_{Q_{2}^{\prime}}$ ). Then the above argument implies $n_{w_{G} w_{Q}} M_{Q_{2}}^{\prime} n_{w_{G} w_{Q}}^{-1}=M_{Q_{2}^{\prime}}^{\prime}$. Hence $n_{w_{G} w_{Q}} W_{\text {aff, } Q_{2}}(1) n_{w_{G} w_{Q}}^{-1}=W_{\text {aff, } Q_{2}^{\prime}}(1)$. Therefore, for $w \in W_{\text {aff, } Q_{2}^{\prime}}(1)$, we have

$$
\left(n_{w_{G} w_{Q}} e_{Q}(\sigma)\right)\left(T_{w}^{Q^{\prime} *}\right)=e_{Q}(\sigma)\left(T_{n_{w_{G} w_{Q}}^{-1} w_{w_{G} w_{Q}}}^{Q *}\right)=1
$$


from the definition of the extension. We get the lemma by the characterization of the extension.

2.13. Supersingular modules. Assume that $p=0$ in $C$. Let $\mathcal{O}$ be a conjugacy class in $W(1)$ which is contained in $\Lambda(1)$. For a spherical orientation $o$, set $z_{\mathcal{O}}=\sum_{\lambda \in \mathcal{O}} E_{o}(\lambda)$. Then this does not depend on $o$ and gives an element of the center of $\mathcal{H}$ [Vig15a, Theorem 5.1]. The length of $\lambda \in \mathcal{O}$ does not depend on $\lambda$. We denote it by $\ell(\mathcal{O})$.

Definition 2.28. Let $\pi$ be an $\mathcal{H}$-module. We call $\pi$ supersingular if there exists $n \in \mathbb{Z}_{>0}$ such that $\pi z_{\mathcal{O}}^{n}=0$ for any $\mathcal{O}$ such that $\ell(\mathcal{O})>0$.

2.14. Simple modules. Assume that $C$ is an algebraically closed field of characteristic $p$. We consider the following triple $(P, \sigma, Q)$.

- $P$ is a parabolic subgroup.

- $\sigma$ is an simple supersingular $\mathcal{H}_{P}$-module.

- $Q$ is a parabolic subgroup between $P$ and $P(\sigma)$.

Define

$$
I(P, \sigma, Q)=I_{P(\sigma)}\left(\operatorname{St}_{Q}^{P(\sigma)}(\sigma)\right) .
$$

Theorem 2.29 ([Abe, Theorem 1.1]). The module $I(P, \sigma, Q)$ is simple and any simple module has this form. Moreover, $(P, \sigma, Q)$ is unique up to isomorphism.

The simple supersingular modules are classified in Oll14, Vig15a. We do not recall the classification since we do not need it in this paper.

\section{A FTILRATION ON PARABOLIC INDUCTIONS}

3.1. A filtration. Let $P$ be a parabolic subgroup and $A$ a subset of $W_{0}^{P}$. For an $\mathcal{H}_{P}$-module $\sigma$, put

$$
I_{P}(\sigma)_{A}=\left\{\varphi \in I_{P}(\sigma) \mid \varphi\left(T_{n_{w}}\right)=0\left(w \in W_{0}^{P} \backslash A\right)\right\} .
$$

We call $A \subset W_{0}^{P}$ open if $v \in A, w \geq v$ implies $w \in A$. Assume that $A$ is open and fix a minimal element $w \in A$. Set $A^{\prime}=A \backslash\{w\}$. Then $A^{\prime}$ is also open. By Proposition 2.9, the map $I_{P}(\sigma)_{A} / I_{P}(\sigma)_{A^{\prime}} \rightarrow \sigma$ given by $\varphi \mapsto \varphi\left(T_{n_{w}}\right)$ is a bijection. In this section, we give a description of the action of $E_{O_{-}}(\lambda)$ on $I_{P}(\sigma)_{A} / I_{P}(\sigma)_{A^{\prime}}$. We start with the following lemma.

Lemma 3.1. Let $w \in W_{P}(1)$ and $\lambda_{0}=\lambda_{P}^{-} \in \Lambda(1)$ as in Proposition 2.5 such that $w \lambda_{0}$ is $P$-negative. Then $q_{w}^{1 / 2} q_{\lambda_{0}}^{1 / 2} q_{w \lambda_{0}}^{-1 / 2}$ does not depend on a choice of $\lambda_{0}$.

Proof. Let $\lambda_{0}^{\prime}$ be another choice and put $\lambda_{1}=\lambda_{0} \lambda_{0}^{\prime}$. Since $\nu\left(\lambda_{0}\right)$ and $\nu\left(\lambda_{0}^{\prime}\right)$ belong to the same closed Weyl chamber, we have $\ell\left(\lambda_{0} \lambda_{0}^{\prime}\right)=\ell\left(\lambda_{0}\right)+$ $\ell\left(\lambda_{0}^{\prime}\right)$ by Lemma 2.11. Hence $q_{\lambda_{1}}=q_{\lambda_{0}} q_{\lambda_{0}^{\prime}}$. By Lemma 2.19 (2), we have $\ell\left(w \lambda_{1}\right)=\ell\left(w \lambda_{0}\right)+\ell\left(\lambda_{0}^{\prime}\right)$. Hence $q_{w \lambda_{1}}=q_{w \lambda_{0}} q_{\lambda_{0}^{\prime}}$. Therefore we get $q_{w}^{1 / 2} q_{\lambda_{0}}^{1 / 2} q_{w \lambda_{0}}^{-1 / 2}=q_{w}^{1 / 2} q_{\lambda_{1}}^{1 / 2} q_{w \lambda_{1}}^{-1 / 2}$. Replacing $\lambda_{0}$ with $\lambda_{0}^{\prime}$, we also have $q_{w}^{1 / 2} q_{\lambda_{0}^{\prime}}^{1 / 2} q_{w \lambda_{0}^{\prime}}^{-1 / 2}=q_{w}^{1 / 2} q_{\lambda_{1}}^{1 / 2} q_{w \lambda_{1}}^{-1 / 2}$. We get the lemma.

We denote $q_{w}^{1 / 2} q_{\lambda_{0}}^{1 / 2} q_{w \lambda_{0}}^{-1 / 2}$ by $q(P, w)$. By Lemma 2.20 , we have $q(P, w)=1$ if and only if $w$ is $P$-negative. 
Proposition 3.2. The subspace $I_{P}(\sigma)_{A}$ is $\mathcal{A}_{o_{-}}$stable and the action of $E_{o_{-}}(\lambda)$ on $I_{P}(\sigma)_{A} / I_{P}(\sigma)_{A^{\prime}} \simeq \sigma$ is given by $q\left(P, n_{w}^{-1} \cdot \lambda\right) E_{o_{-, P}}^{P}\left(n_{w}^{-1} \cdot \lambda\right)$.

We need the following lemma. Recall that we have another basis $\left\{E_{-}(w) \mid\right.$ $w \in W(1)\}$ defined by $E_{-}\left(n_{v} \mu\right)=q_{n_{v}}^{-1 / 2} q_{\mu}^{-1 / 2} q_{n_{v} \mu}^{1 / 2} T_{n_{v}}^{*} E_{o_{-}}(\mu)$ for $v \in W_{0}$ and $\mu \in \Lambda(1)$. From the definition, we have

$$
E_{-}(w) E_{O_{-}}(\lambda)=q_{w}^{1 / 2} q_{\lambda}^{1 / 2} q_{w \lambda}^{-1 / 2} E_{-}(w \lambda) .
$$

Lemma 3.3. Let $X \in \mathcal{H}, \varphi \in I_{P}(\sigma)$ and $w \in W_{P}(1)$. Then we have $\varphi\left(X E_{-}(w)\right)=q(P, w) \varphi(X) \sigma\left(E_{-}^{P}(w)\right)$.

Proof. Replacing $\varphi$ with $\varphi X$, we may assume $X=1$. If $w$ is $P$-negative, then this follows from $q(P, w)=1$ and $j_{P}^{-*}\left(E_{-}^{P}(w)\right)=E_{-}(w)$ [Abe, Lemma 4.6]. In general, let $\lambda_{P}^{-} \in \Lambda(1)$ as in Proposition 2.5 such that $w \lambda_{P}^{-}$is $P$-negative. Then we have

$$
E_{-}(w) E_{o_{-}}\left(\lambda_{P}^{-}\right)=q(P, w) E_{-}\left(w \lambda_{P}^{-}\right) .
$$

Hence we have

$$
\begin{aligned}
\varphi\left(E_{-}(w)\right) & =\varphi\left(E_{-}(w) E_{o_{-}}\left(\lambda_{P}^{-}\right)\right) \sigma\left(E_{o_{-, P}}^{P}\left(\lambda_{P}^{-}\right)^{-1}\right) \\
& =q(P, w) \varphi\left(E_{-}\left(w \lambda_{P}^{-}\right)\right) \sigma\left(E_{o_{-, P}}^{P}\left(\lambda_{P}^{-}\right)^{-1}\right) \\
& =q(P, w) \varphi(1) \sigma\left(E_{-}^{P}\left(w \lambda_{P}^{-}\right) E_{o_{-, P}}^{P}\left(\lambda_{P}^{-}\right)^{-1}\right) \\
& =q(P, w) \varphi(1) \sigma\left(E_{-}^{P}(w)\right)
\end{aligned}
$$

We get the lemma.

We also use:

Lemma 3.4. Let $v \in W_{0}^{P}$ and $\varphi \in I_{P}(\sigma)$. Assume that $\varphi\left(T_{n_{v}}^{*}\right)=0$. Then we have $\varphi\left(E_{-}\left(n_{v} w\right)\right)=0$ for any $w \in W_{P}(1)$.

Proof. Take $\lambda_{P}^{-}$as in Proposition 2.5] such that $w \lambda_{P}^{-}$is $P$-negative. Then we have

$$
\begin{aligned}
\varphi\left(E_{-}\left(n_{v} w\right)\right) & =\varphi\left(E_{-}\left(n_{v} w\right) E_{o_{-}}\left(\lambda_{P}^{-}\right)\right) \sigma\left(E_{o_{-, P}}^{P}\left(\lambda_{P}^{-}\right)^{-1}\right) \\
& \in C\left[q_{s}\right] \varphi\left(E_{-}\left(n_{v} w \lambda_{P}^{-}\right)\right) \sigma\left(E_{o_{-, P}}^{P}\left(\lambda_{P}^{-}\right)^{-1}\right) .
\end{aligned}
$$

Hence it is sufficient to prove $\varphi\left(E_{-}\left(n_{v} w \lambda_{P}^{-}\right)\right)=0$. Namely we may assume $w$ is $P$-negative.

If $w$ is $P$-negative, by Lemma 2.18, we have $\ell\left(n_{v} w\right)=\ell\left(n_{v}\right)+\ell(w)$. Hence by the definition of $E_{-}$, we have $E_{-}\left(n_{v} w\right)=T_{n_{v}}^{*} E_{-}(w)$. Therefore we have $\varphi\left(E_{-}\left(n_{v} w\right)\right)=\varphi\left(T_{n_{v}}^{*}\right) \sigma\left(E_{-}^{P}(w)\right)=0$.

Proof of Proposition 3.2. Let $v \notin A^{\prime}$. By the Bernstein relations Vig16, Corollary 5.43], in $\mathcal{H}\left[q_{s}^{ \pm 1}\right]$, we have

$$
E_{o_{-}}(\lambda) T_{n_{v}} \in T_{n_{v}} E_{o_{-}}\left(n_{v}^{-1} \cdot \lambda\right)+\sum_{v_{1}<v, \mu \in \Lambda(1)} C\left[q_{s}^{ \pm 1}\right] T_{n_{v_{1}}} E(\mu) .
$$


Since $T_{n_{v_{1}}} \in \sum_{v_{2} \leq v_{1}} T_{n_{v_{2}}}^{*} C\left[Z_{\kappa}\right]$, we have

$$
\begin{aligned}
E_{o_{-}}(\lambda) T_{n_{v}} & \in T_{n_{v}} E_{o_{-}}\left(n_{v}^{-1} \cdot \lambda\right)+\sum_{v_{2}<v, \mu \in \Lambda(1)} C\left[q_{s}^{ \pm 1}\right] T_{n_{v_{2}}}^{*} E(\mu) \\
& =T_{n_{v}} E_{o_{-}}\left(n_{v}^{-1} \cdot \lambda\right)+\sum_{v_{2}<v, \mu \in \Lambda(1)} C\left[q_{s}^{ \pm 1}\right] E_{-}\left(n_{v_{2}} \mu\right) .
\end{aligned}
$$

For $v_{2}<v$, take $v_{3} \in W_{0}^{P}$ and $v_{3}^{\prime} \in W_{0, P}$ such that $v_{2}=v_{3} v_{3}^{\prime}$. Then $E_{-}\left(n_{v_{2}} \mu\right)=E_{-}\left(n_{v_{3}} n_{v_{3}^{\prime}} \mu\right)$ and $n_{v_{3}^{\prime}} \mu \in W_{P}(1)$. We have $v_{3} \leq v_{2}<v$. Hence

$$
\begin{aligned}
E_{o_{-}}(\lambda) T_{n_{v}} & \in T_{n_{v}} E_{o_{-}}\left(n_{v}^{-1} \cdot \lambda\right)+\left(\sum_{v_{3}<v, v_{3} \in W_{0}^{P}, x \in W_{P}(1)} C\left[q_{s}^{ \pm 1}\right] E_{-}\left(n_{v_{3}} x\right) \cap \mathcal{H}\right) \\
& =T_{n_{v}} E_{o_{-}}\left(n_{v}^{-1} \cdot \lambda\right)+\sum_{v_{3}<v, v_{3} \in W_{0}^{P}, x \in W_{P}(1)} C\left[q_{s}\right] E_{-}\left(n_{v_{3}} x\right) .
\end{aligned}
$$

Let $\varphi \in I_{P}(\sigma)_{A}$ and we prove $\varphi\left(E_{-}\left(n_{v_{3}} x\right)\right)=0$ for $v_{3}<v, v_{3} \in W_{0}^{P}, x \in$ $W_{P}(1)$ by applying the above lemma. We check $\varphi\left(T_{n_{v_{3}}}^{*}\right)=0$. We have $T_{n_{v_{3}}}^{*} \in \sum_{v_{4} \leq v_{3}} T_{n_{v_{4}}} C\left[Z_{\kappa}\right]$. Since $v_{4} \leq v_{3}<v$ and $v \notin A^{\prime}$, we have $v_{4} \notin A$. Hence $\varphi\left(T_{n_{v_{4}}}\right)=0$. Therefore we get $\varphi\left(T_{n_{v_{3}}}^{*}\right)=0$.

Therefore we have $\left(\varphi E_{O_{-}}(\lambda)\right)\left(T_{n_{v}}\right)=\varphi\left(T_{n_{v}} E_{o_{-}}\left(n_{v}^{-1} \cdot \lambda\right)\right)$. By Lemma 3.3. we have $\varphi\left(T_{n_{v}} E_{o_{-}}\left(n_{v}^{-1} \cdot \lambda\right)\right)=q\left(P, n_{v}^{-1} \cdot \lambda\right) \varphi\left(T_{n_{v}}\right) \sigma\left(E_{o_{-}}^{P}\left(n_{v}^{-1} \cdot \lambda\right)\right)$. This is zero if $v \neq w$. Hence $\varphi E_{o_{-}}(\lambda) \in I_{P}(\sigma)_{A}$. If $v=w$, we get $\varphi\left(E_{o_{-}}(\lambda) T_{n_{w}}\right)=$ $q\left(P, n_{w}^{-1} \cdot \lambda\right) \varphi\left(T_{n_{w}}\right) \sigma\left(E_{o_{-}}^{P}\left(n_{w}^{-1} \cdot \lambda\right)\right)$. This gives the lemma.

Finally, we describe the filtration in terms of a tensor product. Recall that we have an isomorphism (Proposition 2.21)

$$
I_{P}(\sigma) \simeq n_{w_{G} w_{P}} \sigma \otimes_{\left(\mathcal{H}_{P^{\prime}}^{+}, j_{P^{\prime}}^{+}\right)} \mathcal{H}
$$

where $P^{\prime}=n_{w_{G} w_{P}} P^{\mathrm{op}} n_{w_{G} w_{P}}^{-1}$ Let $A \subset P^{\prime} W_{0}$ be a closed subset, namely a subset which satisfies that $v \in A, w \leq v$ implies $w \in A$. Set $A_{0}=$ $\left\{w^{-1} w_{G} w_{P} \mid w \in A\right\}$. Then $A_{0} \subset W_{0}^{P}$ is an open subset by Lemma 2.22 , By Lemma 2.22, $I_{P}(\sigma)_{A_{0}}$ corresponds to

$$
\sum_{v \in A} n_{w_{G} w_{P}} \sigma \otimes T_{n_{v}}^{*} .
$$

Let $w \in A$ be a maximal element and put $A^{\prime}=A \backslash\{w\}$.

Lemma 3.5. The quotient

$$
\left(\sum_{v \in A} n_{w_{G} w_{P}} \sigma \otimes T_{n_{v}}^{*}\right) /\left(\sum_{v \in A^{\prime}} n_{w_{G} w_{P}} \sigma \otimes T_{n_{v}}^{*}\right)
$$

is isomorphic to $\sigma$ as a vector space and the action of $E_{o_{-}}(\lambda)$ is given by

$$
q\left(P, n_{w^{-1} w_{G} w_{P}}^{-1} \cdot \lambda\right) \sigma\left(E_{o_{-, P}}^{P}\left(n_{w^{-1} w_{G} w_{P}}^{-1} \cdot \lambda\right)\right) .
$$


Remark 3.6. Since $\ell(w)+\ell\left(w^{-1} w_{G} w_{P}\right)=\ell\left(w_{G} w_{P}\right)$ (see the last part of the proof of Lemma 2.22), we have $n_{w^{-1} w_{G} w_{P}}=n_{w}^{-1} n_{w_{G} w_{P}}$. Hence we have

$$
\begin{aligned}
\sigma\left(E_{o_{-, P}}^{P}\left(n_{w^{-1} w_{G} w_{P}}^{-1} \cdot \lambda\right)\right) & =\sigma\left(E_{o_{-, P}}^{P}\left(n_{w_{G} w_{P}}^{-1} n_{w} \cdot \lambda\right)\right) \\
& =\left(n_{w_{G} w_{P}} \sigma\right)\left(E_{o_{-, P^{\prime}}}^{P^{\prime}}\left(n_{w} \cdot \lambda\right)\right) .
\end{aligned}
$$

Remark 3.7. For any $\mu, q\left(P, n_{w_{G} w_{P}}^{-1} \cdot \mu\right)=1$ if and only if $n_{w_{G} w_{P}}^{-1} \cdot \mu$ is $P$ negative. Set $P^{\prime}=n_{w_{G} w_{P}} P^{\mathrm{op}} n_{w_{G} w_{P}}^{-1}$. Then we have $\left(w_{G} w_{P}\right)\left(\Sigma^{+} \backslash \Sigma_{P}^{+}\right)=$ $\Sigma^{-} \backslash \Sigma_{P^{\prime}}^{-}$. Hence $q\left(P, n_{w_{G} w_{P}}^{-1} \cdot \mu\right)=1$ if and only if $\mu$ is $P^{\prime}$-positive. Therefore $q\left(P, n_{w^{-1} w_{G} w_{P}}^{-1} \cdot \lambda\right)=1$ if and only if $n_{w} \cdot \lambda$ is $P^{\prime}$-positive.

3.2. Sum and intersections. In this subsection, assume that $P$ is a parabolic subgroup and $\sigma$ an $\mathcal{H}_{P}$-module which has the extension to $\mathcal{H}$. Let $Q$ be a parabolic subgroup containing $P$. Let $A \subset W_{0}^{Q}$ be an open subset. Then we have $I_{Q}(\sigma)_{A} \subset I_{Q}(\sigma)$. In this subsection we prove the following lemma using an argument in $\mathrm{AHVa}$.

Lemma 3.8. Let $\mathcal{P} \subset\left\{Q_{1} \supset Q\right\}$ be a subset. Then we have

$$
I_{Q}\left(e_{Q}(\sigma)\right)_{A} \cap \sum_{Q_{1} \in \mathcal{P}}\left(I_{Q_{1}}\left(e_{Q_{1}}(\sigma)\right)\right)=\sum_{Q_{1} \in \mathcal{P}}\left(I_{Q}\left(e_{Q}(\sigma)\right)_{A} \cap I_{Q_{1}}\left(e_{Q_{1}}(\sigma)\right)\right) .
$$

Remark 3.9. The above lemma is equivalent to the following. Let $\mathcal{P} \subset$ $\left\{Q_{1} \supset Q\right\}$ be a subset and set $\pi=I_{Q}\left(e_{Q}(\sigma)\right) / \sum_{Q_{1} \in \mathcal{P}} I_{Q_{1}}\left(e_{Q_{1}}(\sigma)\right)$. Put $I_{Q_{1}, A}=I_{Q_{1}}\left(e_{Q_{1}}(\sigma)\right) \cap I_{Q}\left(e_{Q}(\sigma)\right)_{A}$ and let $\pi_{A}$ be the image of $I_{Q}\left(e_{Q}(\sigma)\right)_{A}$. Then the sequence

$$
\bigoplus_{Q_{1} \in \mathcal{P}} I_{Q_{1}, A} \rightarrow I_{Q, A} \rightarrow \pi_{A} \rightarrow 0
$$

is exact.

Take a minimal element $w \in A$ and set $A^{\prime}=A \backslash\{w\}$.

Lemma 3.10. Let $Q_{1} \supset Q$. The injective map $I_{Q_{1}, A} / I_{Q_{1}, A^{\prime}} \hookrightarrow I_{Q, A} / I_{Q, A^{\prime}}$ is surjective if $w \in W_{0}^{Q_{1}}$ and 0 otherwise.

Proof. Recall that $\varphi \mapsto \varphi\left(T_{n_{w}}\right)$ gives an isomorphism $I_{Q, A} / I_{Q, A^{\prime}} \simeq \sigma$. Assume that $w \in W_{0}^{Q_{1}}$ and let $\varphi \in I_{Q, A}$. Set $x=\varphi\left(T_{n_{w}}\right)$ and take $\psi \in I_{Q_{1}}\left(e_{Q_{1}}(\sigma)\right)$ such that $\psi\left(T_{n_{w}}\right)=x$ and $\psi\left(T_{n_{v}}\right)=0$ for $v \in W_{0}^{Q_{1}} \backslash\{w\}$. Let $v \in W_{0}^{Q}$ and take $v_{1} \in W_{0}^{Q_{1}}$ and $v_{2} \in W_{Q_{1}, 0}$ such that $v=v_{1} v_{2}$. Then we have $\psi\left(T_{n_{v}}\right)=\psi\left(T_{n_{v_{1}}}\right) e_{Q_{1}}(\sigma)\left(T_{n_{v_{2}}}^{Q_{1}}\right)$ since $j_{Q_{1}}^{-*}\left(T_{n_{v_{2}}}^{Q_{1}}\right)=T_{n_{v_{2}}}$ by Lemma 2.7. Hence if $\psi\left(T_{n_{v}}\right) \neq 0$, then $v_{1}=w$. Therefore $v \in w W_{Q_{1}, 0}$. Since $w \in W_{0}^{Q_{1}}$, any element in $w W_{Q_{1}, 0}$ is greater than or equal to $w$. Hence $v \in A$. Namely $\psi \in I_{Q_{1}, A}$ and we proved the surjectivity of the map in the lemma.

Assume that $w \notin W_{0}^{Q_{1}}$ and take $w_{1} \in W_{0}^{Q_{1}}$ and $w_{2} \in W_{Q_{1}, 0}$ such that $w=w_{1} w_{2}$. Then $w_{1}<w$. Since $w$ is minimal in $A$, we have $w_{1} \notin A$. Hence for $\psi \in I_{Q_{1}, A}$, we have $\psi\left(T_{n_{w_{1}}}\right)=0$. Therefore we have $\psi\left(T_{n_{w}}\right)=$ $\psi\left(T_{n_{w_{1}}}\right) e_{Q_{1}}(\sigma)\left(T_{n_{w_{2}}}^{Q_{1}}\right)=0$. Hence we have $\psi \in I_{Q_{1}, A^{\prime}}$. We get $I_{Q_{1}, A}=$ $I_{Q_{1}, A^{\prime}}$. 
Proof of Lemma 3.8. Obviously the left hand side contains the right hand side. We prove that the right hand side contains the left hand side by backward induction on \#A. We assume that the lemma is true for $A$ and prove the lemma for $A^{\prime}$. By inductive hypothesis, we have

$$
\begin{aligned}
I_{Q, A^{\prime}} \cap \sum_{Q_{1} \in \mathcal{P}} I_{Q_{1}}\left(e_{Q_{1}}(\sigma)\right) & =I_{Q, A^{\prime}} \cap I_{Q, A} \cap \sum_{Q_{1} \in \mathcal{P}} I_{Q_{1}}\left(e_{Q_{1}}(\sigma)\right) \\
& =I_{Q, A^{\prime}} \cap \sum_{Q_{1} \in \mathcal{P}} I_{Q_{1}, A}
\end{aligned}
$$

First assume that $w \notin W_{0}^{Q_{1}}$ for any $Q_{1} \in \mathcal{P}$. Then by the above lemma, we have $I_{Q_{1}, A}=I_{Q_{1}, A^{\prime}}$ for any $Q_{1} \in \mathcal{P}$. Hence

$$
I_{Q, A^{\prime}} \cap \sum_{Q_{1} \in \mathcal{P}} I_{Q_{1}, A}=I_{Q, A^{\prime}} \cap \sum_{Q_{1} \in \mathcal{P}} I_{Q_{1}, A^{\prime}}=\sum_{Q_{1} \in \mathcal{P}} I_{Q_{1}, A^{\prime}}
$$

Now assume that there exists $Q_{1} \in \mathcal{P}$ such that $w \in W_{0}^{Q_{1}}$. Take $\varphi_{Q_{1}} \in$ $I_{Q_{1}, A}$ such that $\sum_{Q_{1} \in \mathcal{P}} \varphi_{Q_{1}} \in I_{Q, A^{\prime}}$. Set $\mathcal{P}_{1}=\left\{Q_{1} \in \mathcal{P} \mid w \in W_{0}^{Q_{1}}\right\}$. Let $Q_{0}$ be a parabolic subgroup corresponding to $\bigcup_{Q_{1} \in \mathcal{P}_{1}} \Delta_{Q_{1}}$. By the above lemma, for each $Q_{1} \in \mathcal{P}_{1}$, we have $I_{Q_{0}, A} / I_{Q_{0}, A^{\prime}} \simeq I_{Q_{1}, A} / I_{Q_{1}, A^{\prime}}$. Therefore for each $Q_{1} \in \mathcal{P}_{1}$ there exists $\varphi_{Q_{1}}^{\prime} \in I_{Q_{0}, A}$ such that $\varphi_{Q_{1}}-\varphi_{Q_{1}}^{\prime} \in I_{Q_{1}, A^{\prime}}$. Then we have

$$
\begin{aligned}
\sum_{Q_{1} \in \mathcal{P}} \varphi_{Q_{1}} & =\sum_{Q_{1} \in \mathcal{P} \backslash \mathcal{P}_{1}} \varphi_{Q_{1}}+\sum_{Q_{1} \in \mathcal{P}_{1}}\left(\varphi_{Q_{1}}-\varphi_{Q_{1}}^{\prime}\right)+\sum_{Q_{1} \in \mathcal{P}_{1}} \varphi_{Q_{1}}^{\prime} \\
& \in \sum_{Q_{1} \in \mathcal{P} \backslash \mathcal{P}_{1}} I_{Q_{1}, A}+\sum_{Q_{1} \in \mathcal{P}} I_{Q_{1}, A^{\prime}}+\sum_{Q_{1} \in \mathcal{P}_{1}} \varphi_{Q_{1}}^{\prime} .
\end{aligned}
$$

By the above lemma, for $Q_{1} \in \mathcal{P} \backslash \mathcal{P}_{1}$, we have $I_{Q_{1}, A}=I_{Q_{1}, A^{\prime}}$. Hence

$$
\sum_{Q_{1} \in \mathcal{P}} \varphi_{Q_{1}} \in \sum_{Q_{1} \in \mathcal{P} \backslash \mathcal{P}_{1}} I_{Q_{1}, A^{\prime}}+\sum_{Q_{1} \in \mathcal{P}_{1}} I_{Q_{1}, A^{\prime}}+\sum_{Q_{1} \in \mathcal{P}_{1}} \varphi_{Q_{1}}^{\prime}
$$

In particular, $\sum_{Q_{1} \in \mathcal{P}_{1}} \varphi_{Q_{1}}^{\prime} \in I_{Q, A^{\prime}} \cap I_{Q_{0}}\left(e_{Q_{0}}(\sigma)\right)=I_{Q_{0}, A^{\prime}}$ since $\sum_{Q_{1} \in \mathcal{P}} \varphi_{Q_{1}} \in$ $I_{Q, A^{\prime}}$. For $Q_{1} \in \mathcal{P}_{1}$, we have $I_{Q_{0}, A^{\prime}} \subset I_{Q_{1}, A^{\prime}}$. Hence $I_{Q_{0}, A^{\prime}} \subset \sum_{Q_{1} \in \mathcal{P}_{1}} I_{Q_{1}, A^{\prime}}$. Therefore

$$
\sum_{Q_{1} \in \mathcal{P}} \varphi_{Q_{1}} \in \sum_{Q_{1} \in \mathcal{P} \backslash \mathcal{P}_{1}} I_{Q_{1}, A^{\prime}}+\sum_{Q_{1} \in \mathcal{P}_{1}} I_{Q_{1}, A^{\prime}}+\sum_{Q_{1} \in \mathcal{P}_{1}} I_{Q_{1}, A^{\prime}}=\sum_{Q_{1} \in \mathcal{P}} I_{Q_{1}, A^{\prime}}
$$

We get the lemma.

3.3. A filtration on generalized Steinberg modules. As in the previous section, let $P$ be a parabolic subgroup and $\sigma$ an $\mathcal{H}_{P}$-module which has the extension to $\mathcal{H}$. Let $Q$ be a parabolic subgroup containing $P$. As in Remark 3.9, for each open subset $A \subset W_{0}^{Q}$, set $I_{Q_{1}, A}=I_{Q_{1}}\left(e_{Q_{1}}(\sigma)\right) \cap$ $I_{Q}\left(e_{Q}(\sigma)\right)_{A}$ and let $\operatorname{St}_{Q, A}$ be the image of $I_{Q}\left(e_{Q}(\sigma)\right)_{A}$. Let $w \in A$ be a minimal element and put $A^{\prime}=A \backslash\{w\}$. Then we have a commutative 
diagram.

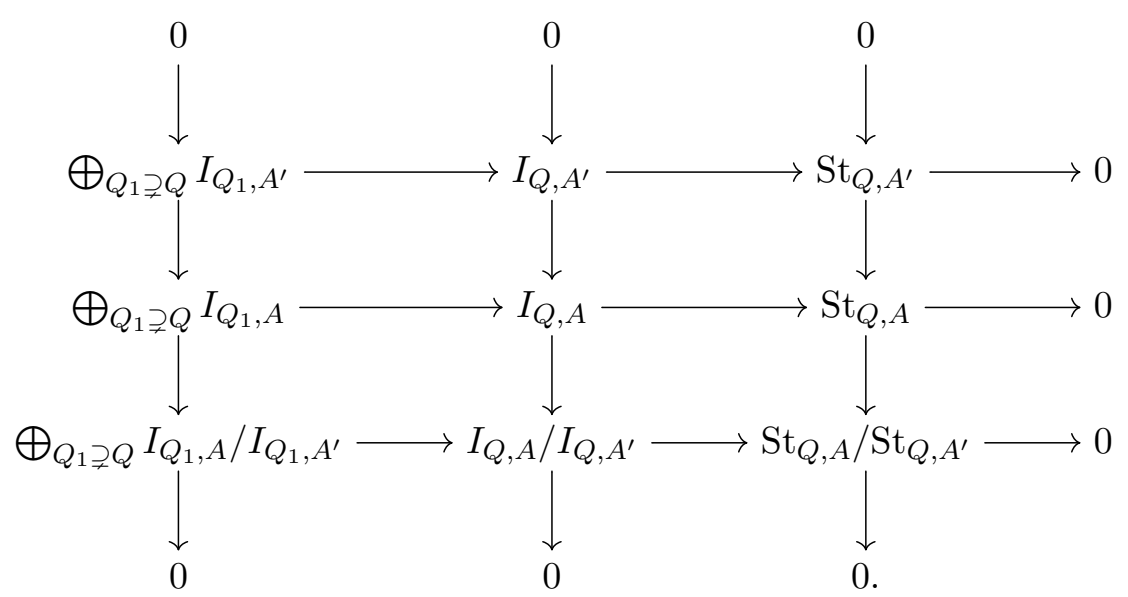

Since the first two rows are exact by Remark 3.9, the third row is also exact. If $w \notin W_{0}^{Q_{1}}$ for any $Q_{1} \supsetneq Q$, we have $I_{Q_{1}, A} / I_{Q_{1}, A^{\prime}}=0$ for any $Q_{1} \supsetneq Q$ by Lemma 3.10 . Hence $I_{Q, A} / I_{Q, A^{\prime}} \stackrel{\sim}{\rightarrow} \mathrm{St}_{Q, A} / \mathrm{St}_{Q, A^{\prime}}$. If $w \in W_{0}^{Q_{1}}$ for some $Q_{1} \supsetneq Q$, we have $I_{Q_{1}, A} / I_{Q_{1}, A^{\prime}} \stackrel{\sim}{\rightarrow} I_{Q, A} / I_{Q, A^{\prime}}$ by Lemma 3.10 . Hence $\bigoplus_{Q_{1} \supsetneq Q} I_{Q_{1}, A} / I_{Q_{1}, A^{\prime}} \rightarrow I_{Q, A} / I_{Q, A^{\prime}}$ is surjective. Therefore we have $\mathrm{St}_{Q, A} / \mathrm{St}_{Q, A^{\prime}}=0$. Summarizing this argument, we get the following lemma.

Lemma 3.11. If $w \in W_{0}^{Q_{1}}$ for some $Q_{1} \supsetneq Q$ then $\mathrm{St}_{Q, A} / \mathrm{St}_{Q, A^{\prime}}=0$. If $w \notin W_{0}^{Q_{1}}$ for any $Q_{1} \supsetneq Q$ then $I_{Q, A} / I_{Q, A^{\prime}} \stackrel{\sim}{\rightarrow} \mathrm{St}_{Q, A} / \mathrm{St}_{Q, A^{\prime}}$.

Using this, we give a description of $\operatorname{St}_{P}(\sigma)$. Let $P_{2}$ be a parabolic subgroup corresponding to $\Delta \backslash \Delta_{P}$. Note that, since $\sigma$ is assumed to be have the extension to $\mathcal{H}, \Delta_{P}$ and $\Delta \backslash \Delta_{P}=\Delta_{P_{2}}$ are orthogonal to each others. Hence $W_{0}^{P}=W_{0, P_{2}}$.

Proposition 3.12. The representation $\pi=\operatorname{St}_{P}(\sigma)$ is isomorphic to $\sigma$ as an $\left(\mathcal{H}_{P}^{+}, j_{P}^{+}\right)$-module and for $w \in W_{\mathrm{aff}, P_{2}}(1), \pi\left(T_{w}\right)=(-1)^{\ell(w)}$.

Proof. Let $w \in W_{0}^{P}$ and assume that $w \notin W_{0}^{Q_{1}}$ for any $Q_{1} \supsetneq P$. Let $\alpha \in$ $\Delta \backslash \Delta_{P}$ and consider the parabolic subgroup $Q_{1}$ corresponding to $\Delta_{P} \cup\{\alpha\}$. Then by the assumption $w\left(\Delta_{Q_{1}}\right) \not \subset \Sigma^{+}$. Since $w\left(\Delta_{P}\right) \subset \Sigma^{+}$, we have $w(\alpha)<0$. Therefore $w \in W_{0}^{P}=W_{0, P_{2}}$ satisfies that $w(\alpha)<0$ for any $\alpha \in \Delta_{P_{2}}$. Hence $w=w_{P_{2}}=w_{G} w_{P}$. Combining the above lemma, we get the following. Note that $\left\{w_{G} w_{P}\right\}$ is open.

- $\operatorname{St}_{P}(\sigma) / \mathrm{St}_{P,\left\{w_{G} w_{P}\right\}}$ has a filtration with zero successive quotients. Hence $\operatorname{St}_{P}(\sigma)=\mathrm{St}_{P,\left\{w_{G} w_{P}\right\}}$.

- $\mathrm{St}_{P,\left\{w_{G} w_{P}\right\}} \simeq I_{P}(\sigma)_{\left\{w_{G} w_{P}\right\}}$.

Set $\sigma^{\prime}=I_{P}(\sigma)_{\left\{w_{G} w_{P}\right\}}=\left\{\varphi \in I_{P}(\sigma) \mid \varphi\left(T_{n_{v}}\right)=0\left(v \in W_{0}^{P} \backslash\left\{w_{G} w_{P}\right\}\right)\right\}$. Then $\sigma^{\prime} \hookrightarrow I_{P}(\sigma) \rightarrow \operatorname{St}_{P}(\sigma)$ is an isomorphism. By Proposition 2.21, $\sigma^{\prime}$ is $j_{P}^{+}\left(\mathcal{H}_{P}^{+}\right)$-stable and isomorphic to $n_{w_{G} w_{P}} \sigma$ as $\left(\mathcal{H}_{P}^{+}, j_{P}^{+}\right)$-modules. By Lemma 2.26, we get the first part of the proposition.

Next, we prove $\pi\left(T_{w}\right)=(-1)^{\ell(w)}$ for $w \in W_{\text {aff, } P_{2}}(1)$ by the following three steps. 
(1) The claim is true for $w=n_{v}$ where $v \in W_{0, P_{2}}$.

(2) For any $v_{1}, v_{2} \in W_{0, P_{2}}$ and $w \in W(1)$, we have $\pi\left(T_{n_{v_{1}} w n_{v_{2}}}\right)=$ $\pi\left(T_{n_{v_{1}}}\right) \pi\left(T_{w}\right) \pi\left(T_{n_{v_{2}}}\right)$.

(3) We have $\pi\left(T_{w}\right)=(-1)^{\ell(w)}$ for any $w \in W_{\mathrm{aff}, P_{2}}(1)$.

(1) We may assume $v=s \in S_{0, P_{2}}$. Let $\bar{\varphi} \in \operatorname{St}_{P}(\sigma)$ and $\varphi \in \sigma^{\prime}$ its lift. For $w \in W_{0}^{P}=W_{0, P_{2}}$, we have

$$
\left(\varphi T_{n_{s}}\right)\left(T_{n_{w}}\right)= \begin{cases}\varphi\left(T_{n_{w_{2}}}\right) & w=s w_{P_{2}}, \\ \left(q_{s}-1\right) \varphi\left(T_{n_{w_{2}}}\right) & w=w_{P_{2}}, \\ 0 & \text { otherwise. }\end{cases}
$$

Indeed, if $w<s w$, then $\left(\varphi T_{n_{s}}\right)\left(T_{n_{w}}\right)=\varphi\left(T_{n_{s w}}\right)$. Hence if $s w \neq w_{P_{2}}$ then this is zero. If $w>s w$, then $\left(\varphi T_{n_{s}}\right)\left(T_{n_{w}}\right)=\varphi\left(T_{n_{s}}^{2} T_{n_{s w}}\right)=\varphi\left(c_{n_{s}} T_{n_{w}}+\right.$ $\left.q_{s} T_{n_{s w}}\right)$. Since $s(s w)>s w, s w \neq w_{P_{2}}$. Hence $\varphi\left(T_{n_{s w}}\right)=0$. Therefore $\left(\varphi T_{n_{s}}\right)\left(T_{n_{w}}\right)=\varphi\left(c_{n_{s}} T_{n_{w}}\right)=\varphi\left(T_{n_{w}}\right) e_{Q}(\sigma)\left(n_{w}^{-1} \cdot c_{n_{s}}\right)$. This is zero if $w \neq w_{P_{2}}$. When $w=w_{P_{2}}$, take $c_{t} \in \mathbb{Z}$ such that $n_{w_{P_{2}}}^{-1} \cdot c_{n_{s}}=\sum_{t} c_{t} T_{t}$. Since $n_{w_{P_{2}}}^{-1} \cdot c_{n_{s}} \in$ $C\left[Z_{\kappa} \cap W_{\text {aff, } P_{2}}(1)\right]$ (Lemma 2.3),$c_{t} \neq 0$ implies $t \in Z_{\kappa} \cap W_{\text {aff, } P_{2}}(1)$. Hence $e_{Q}(\sigma)\left(T_{t}\right)=1$ for such $t$. Therefore $e_{Q}(\sigma)\left(n_{w_{P_{2}}}^{-1} \cdot c_{n_{s}}\right)=\sum_{t} c_{t}=q_{s}-1$ by Proposition 2.1. We get the above calculation.

Take $\alpha \in \Delta_{P_{2}}$ such that $s=s_{\alpha}$ and put $\alpha^{\prime}=-w_{P_{2}}(\alpha)$ and $s^{\prime}=s_{\alpha^{\prime}}$. Let $Q_{1}$ be a parabolic subgroup corresponding to $\Delta_{P} \cup\left\{\alpha^{\prime}\right\}$. Then $w_{Q_{1}}=$ $w_{P} s^{\prime}$ and $w_{G} w_{Q_{1}}=w_{P_{2}} s^{\prime}$. Define $\psi \in I_{Q_{1}}\left(e_{Q_{1}}(\sigma)\right)$ by $\psi\left(T_{n_{w}}\right)=0$ for $w \in W_{0}^{Q_{1}} \backslash\left\{w_{P_{2}} s^{\prime}\right\}$ and $\psi\left(T_{n_{w_{P_{2}} s^{\prime}}}\right)=\varphi\left(T_{n_{w_{P_{2}}}}\right)$. We prove

$$
\psi\left(T_{n_{w}}\right)= \begin{cases}\varphi\left(T_{n_{w_{P_{2}}}}\right) & w=s w_{P_{2}}, \\ q_{s} \varphi\left(T_{n_{w_{P_{2}}}}\right) & w=w_{P_{2}}, \\ 0 & \text { otherwise. }\end{cases}
$$

Let $w \in W_{0}^{P}=W_{0, P_{2}}$ and take $w_{1} \in W_{0}^{Q_{1}}$ and $w_{2} \in W_{0, Q_{1} \cap P_{2}}$ such that $w=w_{1} w_{2}$. Then we have $\psi\left(T_{n_{w}}\right)=\psi\left(T_{n_{w_{1}}}\right) e_{Q_{1}}\left(T_{n_{w_{2}}}^{Q_{1}}\right)$. Hence if $w_{1} \neq w_{P_{2}} s^{\prime}$, namely $w \notin w_{P_{2}} s^{\prime} W_{0, Q_{1} \cap P_{2}}$, we have $\psi\left(T_{n_{w}}\right)=0$. Note that $W_{0, Q_{1} \cap P_{2}}=$ $\left\{1, s^{\prime}\right\}$ and $w_{P_{2}} s^{\prime}=s w_{P_{2}}$. Therefore $\psi\left(T_{n_{w}}\right)=0$ if $w \neq w_{P_{2}}, s w_{P_{2}}$. We have $\psi\left(T_{n_{w_{P_{2}}}}\right)=\psi\left(T_{n_{w_{P_{2}} s^{\prime}}}\right) e_{Q_{1}}(\sigma)\left(T_{n_{s^{\prime}}}^{Q_{1}}\right)$ and $e_{Q_{1}}(\sigma)\left(T_{n_{s^{\prime}}}^{Q_{1}}\right)=e_{Q_{1}}(\sigma)\left(T_{n_{s^{\prime}}}^{Q_{1 *}}+\right.$ $\left.c_{n_{s^{\prime}}}\right)=1+q_{s^{\prime}}-1=q_{s^{\prime}}$. Since $s$ and $s^{\prime}$ is conjugate, $q_{s}=q_{s^{\prime}}$. Hence $\psi\left(T_{n_{w_{2}}}\right)=q_{s} \varphi\left(T_{n_{w_{P_{2}}}}\right)$. Finally we have $\psi\left(T_{n_{s w_{P_{2}}}}\right)=\varphi\left(T_{n_{w_{P_{2}}}}\right)$ by the definition of $\psi$. We get the above formula.

Therefore $\left(\varphi T_{n_{s}}-\psi\right)\left(T_{n_{w}}\right)=0=-\varphi\left(T_{n_{w}}\right)$ if $w \neq w_{P_{2}}$ and equal to $-\varphi\left(T_{n_{w_{2}}}\right)$ if $w=w_{P_{2}}$. Since an element in $I_{P}(\sigma)$ is determined by the value at $W_{0}^{P}=W_{0, P_{2}}$, we have $\varphi T_{n_{s}}-\psi=-\varphi$. Hence $\bar{\varphi} T_{n_{s}}=-\bar{\varphi}$ in $\operatorname{St}_{P}(\sigma)$.

(2) Assume that $v_{2}=1$. To prove (2), by induction on the length of $v_{1}$, we may assume $v_{1}=s \in S_{0}$. If $n_{s} w>w$, then it is obvious. Assume that $n_{s} w<w$. Then we have $\pi\left(T_{w}\right)=\pi\left(T_{n_{s}^{-1}} T_{n_{s} w}\right)=\pi\left(T_{n_{s}^{-1}}\right) \pi\left(T_{n_{s} w}\right)=$ $-\pi\left(T_{n_{s} w}\right)$ by (1). Hence $\pi\left(T_{n_{s} w}\right)=-\pi\left(T_{w}\right)=\pi\left(T_{n_{s}}\right) \pi\left(T_{w}\right)$. The same argument implies (2) for any $v_{2}$.

(3) Take $w \in W_{\text {aff }, P_{2}}(1)$. Then we can take $w_{1}, w_{2} \in W_{0, P_{2}}$ and $\lambda \in$ $\Lambda(1) \cap W_{\text {aff, } P_{2}}(1)$ such that $w=n_{w_{1}} \lambda n_{w_{2}}$ and $\lambda$ is anti-dominant with respect 
to $\Sigma_{P_{2}}^{+}$. Since $\Delta_{P}$ is orthogonal to $\Delta_{P_{2}}, \Sigma_{P_{2}}^{+}=\Sigma^{+} \backslash \Sigma_{P}^{+}$. Hence $\lambda$ is $P$ positive. As in Remark 2.25, $\sigma\left(T_{\lambda}^{P}\right)=1$.

Since $\lambda \in \Lambda(1) \cap W_{\text {aff }}(1), \ell(\lambda)$ is even by Lemma [2.14, On the other hand, since $\lambda$ is $P$-positive, we have $\pi\left(T_{\lambda}\right)=\sigma\left(T_{\lambda}^{P}\right)$. Therefore $\pi\left(T_{\lambda}\right)=1=$ $(-1)^{\ell(\lambda)}$. Using (2), we have

$$
\pi\left(T_{w}\right)=\pi\left(T_{n_{w_{1}}}\right) \pi\left(T_{\lambda}\right) \pi\left(T_{n_{w_{2}}}\right)=(-1)^{\ell\left(w_{1}\right)+\ell(\lambda)+\ell\left(w_{2}\right)}=(-1)^{\ell(w)} .
$$

We get the proposition.

3.4. Tensor product decomposition. For the content of this subsection, see also [AHVa]. We start with the following lemma.

Lemma 3.13. Let $P_{1}, P_{2}$ be parabolic subgroups such that $\Delta_{P_{1}}$ is orthogonal to $\Delta_{P_{2}}$ and $\Delta_{P_{1}} \cup \Delta_{P_{2}}=\Delta$. Assume that an $\mathcal{H}_{P_{i}}$-module $\sigma_{i}$ has the extension to $\mathcal{H}$. Then we have the unique action of $\mathcal{H}$ on $e_{G}\left(\sigma_{1}\right) \otimes e_{G}\left(\sigma_{2}\right)$ which satisfies $\left(x_{1} \otimes x_{2}\right) T_{w}^{*}=x_{1} T_{w}^{*} \otimes x_{2} T_{w}^{*}$ for $w \in W(1), x_{1} \in e_{G}\left(\sigma_{1}\right)$ and $x_{2} \in e_{G}\left(\sigma_{2}\right)$.

Proof. The action obviously satisfies the braid relations. It is sufficient to prove that the action satisfies the quadratic relations.

Let $s \in W_{\text {aff }}(1)$ be a lift of a simple reflection. If $s \in W_{P_{1}}(1)$, then $s \in W_{\text {aff }, P_{1}}(1)$. Hence $e_{G}\left(\sigma_{2}\right)\left(T_{s}^{*}\right)=1$. Therefore $x_{1} T_{s}^{*} \otimes x_{2} T_{s}^{*}=x_{1} T_{s}^{*} \otimes x_{2}$. Hence it satisfies the quadratic relations. The quadratic relations hold too if $s \in W_{P_{2}}(1)$.

Remark 3.14. By the above proof, we have $\left(x_{1} \otimes x_{2}\right) T_{w}^{*}=x_{1} T_{w}^{*} \otimes x_{2}$ if $w \in$ $W_{P_{1} \text {,aff }}(1)$. Hence $\left(x_{1} \otimes x_{2}\right) X=x_{1} X \otimes x_{2}$ for any $X \in \bigoplus_{w \in W_{P_{1}, \text { aff }}(1)} C T_{w}^{*}$. In particular, for $X=T_{w}$ or $X=E_{o}(w)$ for any $w \in W_{P_{1} \text {,aff }}(1)$ and any orientation $o$. We also have $\left(x_{1} \otimes x_{2}\right) X=x_{1} \otimes x_{2} X$ for any $X=T_{w}$ or $X=E_{o}(w)$ where $w \in W_{P_{2}, \text { aff }}(1)$.

In the rest of this subsection, let $P$ be a parabolic subgroup, $\sigma$ an $\mathcal{H}_{P^{-}}$ module which has the extension to $\mathcal{H}$. Take a parabolic subgroup $Q$ containing $P$. Let $P_{2}$ be the parabolic subgroup corresponding to $\Delta \backslash \Delta_{P}$. Note that $\Delta_{P_{2}}$ is orthogonal to $\Delta_{P}$.

Lemma 3.15. We have $I_{Q}(\mathbf{1}) \simeq e_{G}\left(I_{P \cap Q}^{P_{2}}(\mathbf{1})\right)$. More generally, for an $\mathcal{H}_{P_{2} \cap Q}$-module $\sigma$ which has an extension to $\mathcal{H}_{Q}$, we have $I_{Q}\left(e_{Q}(\sigma)\right) \simeq$ $e_{G}\left(I_{P_{2} \cap Q}^{P_{2}}(\sigma)\right)$.

We use the following lemma.

Lemma 3.16. Let $P, Q$ be parabolic subgroups.

(1) Let $\lambda_{P}^{-}$as in Proposition 2.5. Then $\mathcal{H}_{P \cap Q}^{P-} \simeq \mathcal{H}_{P \cap Q}^{-}\left(T_{\lambda_{P}^{-}}^{P \cap Q}\right)^{-1}$.

(2) Let $\lambda_{P}^{+}$as in Proposition 2.5. Then $\mathcal{H}_{P \cap Q}^{P+} \simeq \mathcal{H}_{P \cap Q}^{+}\left(T_{\lambda_{P}^{+}}^{P \cap Q}\right)^{-1}$.

Proof. We only prove the first statement. The proof of the second statement is the same.

Let $\lambda \in \Lambda(1)$ such that $\langle\alpha, \nu(\lambda)\rangle \geq 0$ for any $\alpha \in \Sigma_{P}^{+} \backslash \Sigma_{P \cap Q}^{+}$. We can take $n \in \mathbb{Z}_{>0}$ such that $\left\langle\alpha, \nu\left(\lambda\left(\lambda_{P}^{-}\right)^{n}\right)\right\rangle \geq 0$ for any $\alpha \in \Sigma^{+} \backslash \Sigma_{P}^{+}$. Since $\left\langle\alpha, \nu\left(\left(\lambda_{P}^{-}\right)^{n}\right)\right\rangle=0$ for any $\alpha \in \Sigma_{P}^{+}$, we have $\left\langle\alpha, \nu\left(\lambda\left(\lambda_{P}^{-}\right)^{n}\right)\right\rangle=\langle\alpha, \nu(\lambda)\rangle \geq 0$ for any $\alpha \in \Sigma_{P}^{+} \backslash \Sigma_{P \cap Q}^{+}$. Hence $\left\langle\alpha, \nu\left(\lambda\left(\lambda_{P}^{-}\right)^{n}\right)\right\rangle \geq 0$ for any $\alpha \in \Sigma^{+} \backslash \Sigma_{P \cap Q}^{+}$. 
Therefore for any $w \in W_{P \cap Q}(1)$ which is $(P \cap Q)$-negative in $P$, there exists $n \in \mathbb{Z}_{\geq 0}$ such that $w\left(\lambda_{P}^{-}\right)^{n}$ is $(P \cap Q)$-negative in $G$. Then we have $E_{o_{-}, P \cap Q}^{P \cap Q}(w)=E_{o_{-}, P \cap Q}^{P \cap Q}\left(w\left(\lambda_{P}^{-}\right)^{n}\right)\left(T_{\lambda_{P}^{-}}^{P \cap Q}\right)^{-n} \in \mathcal{H}_{P \cap Q}^{-}\left(T_{\lambda_{P}-}^{P \cap Q}\right)^{-1}$.

Proof of Lemma 3.15. Consider the map

$$
I_{Q}\left(e_{Q}(\sigma)\right)=\operatorname{Hom}_{\left(\mathcal{H}_{Q}^{-}, j_{Q}^{-*}\right)}\left(\mathcal{H}, e_{Q}(\sigma)\right) \rightarrow \operatorname{Hom}_{\left(\mathcal{H}_{P_{2} \cap Q}^{-}, j_{P_{2} \cap Q}^{P_{2}-*}\right)}\left(\mathcal{H}_{P_{2}}^{-}, \sigma\right)
$$

defined by $\varphi \mapsto \varphi \circ j_{P_{2}}^{-*}$. Then obviously this is $\left(\mathcal{H}_{P_{2}}^{-}, j_{P_{2}}^{-*}\right)$-equivariant.

Let $\lambda_{P_{2}}^{-}$as in Proposition 2.5. Then we have $\mathcal{H}_{P_{2}}^{-}\left(T_{\lambda_{P_{2}}}^{P_{2}}\right)^{-1}=\mathcal{H}_{P_{2}}$. By Lemma 3.16, we have $\mathcal{H}_{P_{2} \cap Q}^{-}\left(T_{\lambda_{P_{2}}}^{P_{2} \cap Q}\right)^{-1}=\mathcal{H}_{P_{2} \cap Q}^{P_{2}-}$. Therefore we have

$$
I_{P_{2} \cap Q}^{P_{2}}(\sigma)=\operatorname{Hom}_{\left(\mathcal{H}_{P_{2} \cap Q}^{P_{2}-}, j_{P_{2} \cap Q}^{P_{2}-*}\right)}\left(\mathcal{H}_{P_{2}}, \sigma\right)=\operatorname{Hom}_{\left(\mathcal{H}_{P_{2} \cap Q}^{-}, j_{P_{2} \cap Q}^{P_{2}-*}\right)}\left(\mathcal{H}_{P_{2}}^{-}, \sigma\right) .
$$

Hence we get an $\left(\mathcal{H}_{P_{2}}^{-}, j_{P_{2}}^{-*}\right)$-homomorphism $I_{Q}\left(e_{Q}(\sigma)\right) \rightarrow I_{P_{2} \cap Q}^{P_{2}}(\sigma)$. For $v \in W_{0}^{Q}=W_{0, P_{2}}^{P_{2} \cap Q}$, we have $\varphi\left(T_{n_{v}}\right)=\left(\varphi \circ j_{P_{2}}^{-*}\right)\left(T_{n_{v}}^{P_{2}}\right)$ by Corollary 2.7. Hence this homomorphism is an isomorphism by Proposition 2.9.

Let $w \in W_{P, \text { aff }}(1), v \in W_{0}^{Q}$ and $\varphi \in I_{Q}\left(e_{Q}(\sigma)\right)$. We have $W_{0}^{Q} \subset W_{0, P_{2}}$. Since $n_{v} \in W_{P_{2} \text {,aff }}(1), \ell\left(w n_{v}\right)=\ell(w)+\ell\left(n_{v}\right)$. The subgroup $W_{P, \text { aff }}(1)$ is normal in $W(1)$ AHHV, II.7 Remark 4]. Hence $n_{v}^{-1} w n_{v} \in W_{P, \text { aff }}(1)$ and $\ell\left(w n_{v}\right)=\ell\left(n_{v}\right)+\ell\left(n_{v}^{-1} w n_{v}\right)$. Therefore $\left(\varphi T_{w}^{*}\right)\left(T_{n_{v}}^{*}\right)=\varphi\left(T_{w}^{*} T_{n_{v}}^{*}\right)=$ $\varphi\left(T_{w n_{v}}^{*}\right)=\varphi\left(T_{n_{v}}^{*} T_{n_{v}^{-1} w_{v}}^{*}\right)=\varphi\left(T_{n_{v}}^{*}\right) e_{Q}(\sigma)\left(T_{n_{v}^{-1} w n_{v}}^{Q *}\right)$. By the definition of the extension, $e_{Q}(\sigma)\left(T_{n_{v}^{-1} w n_{v}}^{Q *}\right)=1$. Hence $\left(\varphi T_{w}^{*}\right)\left(T_{n_{v}}^{*}\right)=\varphi\left(T_{n_{v}}^{*}\right)$. Since an element in $I_{Q}\left(e_{Q}(\sigma)\right)$ is determined by the values at $T_{n_{v}}^{*}$ for $v \in W_{0}^{Q}$ (Proposition [2.9), $T_{w}^{*}$ acts trivially on $I_{Q}\left(e_{Q}(\sigma)\right)$. Hence $I_{Q}\left(e_{Q}(\sigma)\right) \simeq e_{G}\left(I_{P_{2} \cap Q}^{P_{2}}(\sigma)\right)$.

By this lemma and Lemma 3.13, we have an $\mathcal{H}$-module structure on $I_{Q}(\mathbf{1}) \otimes e_{G}(\sigma)$.

Proposition 3.17. We have $I_{Q}\left(e_{Q}(\sigma)\right) \simeq I_{Q}(\mathbf{1}) \otimes e_{G}(\sigma)$.

Proof. Define the homomorphism $I_{Q}(\mathbf{1}) \otimes e_{G}(\sigma) \rightarrow e_{Q}(\sigma)$ by $\varphi \otimes x \mapsto \varphi(1) x$. For $w \in W_{Q}^{-}(1)$, we have

$$
\begin{aligned}
(\varphi \otimes x) T_{w}^{*}=\varphi T_{w}^{*} \otimes x e_{G}(\sigma)\left(T_{w}^{*}\right) & \mapsto\left(\varphi T_{w}^{*}\right)(1) x e_{G}(\sigma)\left(T_{w}^{*}\right) \\
& =\varphi(1) \mathbf{1}\left(T_{w}^{Q *}\right) x e_{G}(\sigma)\left(T_{w}^{*}\right) \\
& =\varphi(1) x e_{G}(\sigma)\left(T_{w}^{*}\right) .
\end{aligned}
$$

By [Abe, Proposition 4.19], we have $e_{G}(\sigma)\left(T_{w}^{*}\right)=e_{Q}(\sigma)\left(T_{w}^{Q *}\right)$. Hence the homomorphism is $\left(\mathcal{H}_{Q}^{-}, j_{Q}^{-*}\right)$-equivariant. Therefore we get a homomorphism $I_{Q}(\mathbf{1}) \otimes e_{G}(\sigma) \rightarrow I_{Q}\left(e_{Q}(\sigma)\right)$. By Proposition 2.9, we have the decompositions $I_{Q}(\mathbf{1}) \otimes e_{G}(\sigma)=\bigoplus_{w \in W_{0}^{Q}} C \otimes e_{G}(\sigma)$ and $I_{Q}\left(e_{Q}(\sigma)\right)=\bigoplus_{w \in W_{0}^{Q}} e_{Q}(\sigma)$ which 
makes the following diagram commutative

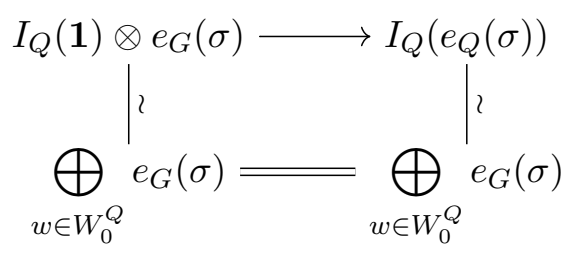

Hence we get the proposition.

\section{INDUCTIONS}

Let $P$ be a parabolic subgroup. Recall that our parabolic induction $I_{P}$ is defined by

$$
I_{P}(\sigma)=\operatorname{Hom}_{\left(\mathcal{H}_{P}^{-}, j_{P}^{-*}\right)}(\mathcal{H}, \sigma)
$$

for an $\mathcal{H}_{P}$-module $\sigma$. Since we also have two subalgebras $\mathcal{H}_{P}^{ \pm}$and four homomorphisms $j_{P}^{ \pm}, j_{P}^{ \pm *}$, we can define four "inductions". In this section, we study the relations between such functors.

4.1. Modules $\sigma_{\ell-\ell_{P}}$. Before studying such functors, we first consider the representation $\sigma_{\ell-\ell_{P}}$ attached to a representation $\sigma$ of $\mathcal{H}_{P}$ where $P$ is a parabolic subgroup.

Let $P$ be a parabolic subgroup and $\sigma$ an $\mathcal{H}_{P}$-module. We define a linear map $\sigma_{\ell-\ell_{P}}$ by

$$
\sigma_{\ell-\ell_{P}}\left(T_{w}^{P}\right)=(-1)^{\ell(w)-\ell_{P}(w)} \sigma\left(T_{w}^{P}\right)
$$

for $w \in W_{P}(1)$. From the following lemma, this is again an $\mathcal{H}_{P}$-module.

Lemma 4.1. (1) The linear map $\mathcal{H}_{P} \rightarrow C$ defined by $T_{w}^{P} \mapsto(-1)^{\ell(w)}$ is a character of $\mathcal{H}_{P}$ and it sends $T_{s}$ to -1 for any $s \in S_{\mathrm{aff}, P}(1)$.

(2) Let $\pi$ be an $\mathcal{H}$-module, $\chi$ a character of $\mathcal{H}$ such that $\chi\left(T_{t}\right)=1$ for any $t \in Z_{\kappa}$ and $\chi\left(T_{s}\right)=-1$ for any $s \in S_{\text {aff }}(1)$. Then the linear map defined by $T_{w} \mapsto(-1)^{\ell(w)} \chi\left(T_{w}\right) \pi\left(T_{w}\right)$ is an $\mathcal{H}$-module.

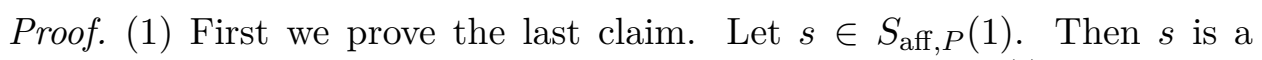
reflection in $W$. Hence $\ell(s)$ is odd. Therefore we have $(-1)^{\ell(s)}=-1$.

We check the quadratic relations. Let $s \in S_{\text {aff, } P}(1)$. The quadratic relation is $\left(T_{s}^{P}\right)^{2}=q_{s, P} T_{s^{2}}^{P}+c_{s} T_{s}^{P}$ and the left hand side goes to 1 by the map in the lemma. Since $s^{2} \in Z_{\kappa}, T_{s^{2}}^{P}$ goes to 1 . Hence $q_{s, P} T_{s^{2}}^{P}$ goes to $q_{s, P}$. The image of $c_{s}$ under the map is $q_{s, P}-1$ by Proposition 2.1. We have already proved that $T_{s}^{P}$ goes to -1 . Hence the right hand side goes to $q_{s, P}+\left(q_{s, P}-1\right)(-1)=1$. Therefore the map preserves the quadratic relations.

We check the braid relations. Let $w_{1}, w_{2} \in W_{P}(1)$. Then $T_{w_{1} w_{2}}^{P}$ goes to $(-1)^{\ell\left(w_{1} w_{2}\right)}=(-1)^{\ell\left(w_{1}\right)+\ell\left(w_{2}\right)}$ which is the product of the images of $T_{w_{1}}^{P}$ and $T_{w_{2}}^{P}$. Hence the map preserves the braid relations. We get (1).

(2) Let $\pi^{\prime}$ be the map given in (21) and first we check that $\pi^{\prime}$ preserves the quadratic relations. Let $s \in S_{\text {aff }}(1)$. We prove $\pi^{\prime}\left(T_{s}\right)^{2}=\pi^{\prime}\left(q_{s} T_{s^{2}}\right)+\pi^{\prime}\left(c_{s} T_{s}\right)$. We calculate the right hand side. Since $s^{2} \in Z_{\kappa}$, we have $\chi\left(T_{s^{2}}\right)=1$. We also have $\ell\left(s^{2}\right)=0$. Hence $\pi^{\prime}\left(T_{s^{2}}\right)=\pi\left(T_{s^{2}}\right)$. Take $c_{s}(t) \in \mathbb{Z}$ such 
that $c_{s}=\sum_{t \in Z_{\kappa}} c_{s}(t) T_{t}$. Then we have $\pi^{\prime}\left(c_{s} T_{s}\right)=\sum_{t \in Z_{\kappa}} c_{s}(t) \pi^{\prime}\left(T_{t} T_{s}\right)=$ $\sum_{t \in Z_{\kappa}} c_{s}(t)(-1)^{\ell(t s)} \chi\left(T_{t}\right) \chi\left(T_{s}\right) \pi\left(T_{t}\right) \pi\left(T_{s}\right)$. Since $t \in Z_{\kappa}$, we have $\ell(t s)=$ $\ell(s)=1$. We also have $\chi\left(T_{t}\right)=1$ by the assumption. Hence $\pi^{\prime}\left(c_{s} T_{s}\right)=$ $-\chi\left(T_{s}\right) \sum_{t \in Z_{\kappa}} c_{s}(t) \pi\left(T_{t}\right) \pi\left(T_{s}\right)=-\chi\left(T_{s}\right) \pi\left(c_{s}\right) \pi\left(T_{s}\right)$. Therefore $\pi^{\prime}\left(q_{s} T_{s^{2}}\right)+$ $\pi^{\prime}\left(c_{s} T_{s}\right)=q_{s} \pi\left(T_{s^{2}}\right)-\chi\left(T_{s}\right) \pi\left(c_{s}\right) \pi\left(T_{s}\right)$. Since we assume $\chi\left(T_{s}\right)=-1$, we get $q_{s} \pi\left(T_{s^{2}}\right)-\chi\left(T_{s}\right) \pi\left(c_{s}\right) \pi\left(T_{s}\right)=q_{s} \pi\left(T_{s^{2}}\right)+\pi\left(c_{s}\right) \pi\left(T_{s}\right)=\pi\left(q_{s} T_{s^{2}}+c_{s} T_{s}\right)=$ $\pi\left(T_{s}^{2}\right)=\pi\left(T_{s}\right)^{2}$. We have $\pi^{\prime}\left(T_{s}\right)^{2}=\chi\left(T_{s}\right)^{2} \pi\left(T_{s}\right)^{2}$. By the assumption, $\chi\left(T_{s}\right)=-1$. Hence $\pi^{\prime}\left(T_{s}\right)^{2}=\pi\left(T_{s}\right)^{2}$. We get the quadratic relations.

Let $w_{1}, w_{2} \in W_{P}(1)$ such that $\ell\left(w_{1} w_{2}\right)=\ell\left(w_{1}\right)+\ell\left(w_{2}\right)$. Then

$$
\begin{aligned}
\pi^{\prime}\left(T_{w_{1}} T_{w_{2}}\right) & =(-1)^{\ell\left(w_{1} w_{2}\right)} \chi\left(T_{w_{1} w_{2}}\right) \pi\left(T_{w_{1} w_{2}}\right) \\
& =(-1)^{\ell\left(w_{1}\right)+\ell\left(w_{2}\right)} \chi\left(T_{w_{1}} T_{w_{2}}\right) \pi\left(T_{w_{1}} T_{w_{2}}\right) \\
& =(-1)^{\ell\left(w_{1}\right)}(-1)^{\ell\left(w_{2}\right)} \chi\left(T_{w_{1}}\right) \chi\left(T_{w_{2}}\right) \pi\left(T_{w_{1}}\right) \pi\left(T_{w_{2}}\right) .
\end{aligned}
$$

Since $\chi\left(T_{w_{2}}\right)$ is a scalar, we have

$$
\begin{aligned}
& (-1)^{\ell\left(w_{1}\right)}(-1)^{\ell\left(w_{2}\right)} \chi\left(T_{w_{1}}\right) \chi\left(T_{w_{2}}\right) \pi\left(T_{w_{1}}\right) \pi\left(T_{w_{2}}\right) \\
& =(-1)^{\ell\left(w_{1}\right)} \chi\left(T_{w_{1}}\right) \pi\left(T_{w_{1}}\right)(-1)^{\ell\left(w_{2}\right)} \chi\left(T_{w_{2}}\right) \pi\left(T_{w_{2}}\right) \\
& =\pi^{\prime}\left(T_{w_{1}}\right) \pi^{\prime}\left(T_{w_{2}}\right) .
\end{aligned}
$$

Hence $\pi^{\prime}$ preserves the braid relations.

Let $\iota=\iota_{G}: \mathcal{H} \rightarrow \mathcal{H}$ be a linear map defined by $T_{w} \mapsto(-1)^{\ell(w)} T_{w}^{*}$ for $w \in W(1)$. Then this is an involution [Vig16, Proposition 4.23]. For any $\mathcal{H}$-module $\pi$, set $\pi^{\iota}=\pi \circ \iota$.

Lemma 4.2. We have $\left(\sigma^{\iota^{P}}\right)_{\ell-\ell_{P}}=\left(\sigma_{\ell-\ell_{P}}\right)^{\iota_{P}}$.

Proof. We prove $\left(\sigma^{\iota_{P}}\right)_{\ell-\ell_{P}}\left(T_{w}^{P}\right)=\left(\sigma_{\ell-\ell_{P}}\right)^{\iota_{P}}\left(T_{w}^{P}\right)$ for any $w \in W_{P}(1)$. We may assume that $w \in S_{\text {aff, } P}(1)$ or $\ell_{P}(w)=0$.

First assume that $w \in S_{\text {aff, } P}(1)$ and denote $w$ by $s$. By Lemma 4.1 (1), we have $(-1)^{\ell(s)}=-1$. Hence $\left(\sigma^{\iota_{P}}\right)_{\ell-\ell_{P}}\left(T_{s}^{P}\right)=(-1)^{\ell(s)-\ell_{P}(s)} \sigma\left(\iota_{P}\left(T_{s}^{P}\right)\right)=$ $\sigma\left(\iota_{P}\left(T_{s}^{P}\right)\right)=-\sigma\left(T_{s}^{P *}\right)$. On the other hand, we have $\left(\sigma_{\ell-\ell_{P}}\right)^{\iota_{P}}\left(T_{s}^{P}\right)=$ $-\left(\sigma_{\ell-\ell_{P}}\right)\left(T_{s}^{P *}\right)=-\left(\sigma_{\ell-\ell_{P}}\right)\left(T_{s}^{P}\right)+\left(\sigma_{\ell-\ell_{P}}\right)\left(c_{s}\right)$. We have $\left(\sigma_{\ell-\ell_{P}}\right)\left(T_{s}^{P}\right)=$ $(-1)^{\ell(s)-\ell_{P}(s)} \sigma\left(T_{s}^{P}\right)=\sigma\left(T_{s}^{P}\right)$ since $(-1)^{\ell(s)}=-1$ and $\ell_{P}(s)=1$. We have $\left(\sigma_{\ell-\ell_{P}}\right)\left(c_{s}\right)=\sigma\left(c_{s}\right)$. Hence $-\left(\sigma_{\ell-\ell_{P}}\right)\left(T_{s}^{P}\right)+\left(\sigma_{\ell-\ell_{P}}\right)\left(c_{s}\right)=-\sigma\left(T_{s}^{P}-c_{s}\right)=$ $-\sigma\left(T_{s}^{P *}\right)$.

Next assume that $\ell_{P}(w)=0$. Then $\iota_{P}\left(T_{w}^{P}\right)=(-1)^{\ell_{P}(w)} T_{w}^{P *}=T_{w}^{P}$. Hence we have $\left(\sigma_{\ell-\ell_{P}}\right)^{\iota_{P}}=\sigma_{\ell-\ell_{P}}\left(T_{w}^{P}\right)=(-1)^{\ell(w)-\ell_{P}(w)} \sigma\left(T_{w}^{P}\right)$. We also have $\left(\sigma^{\iota_{P}}\right)_{\ell-\ell_{P}}\left(T_{w}^{P}\right)=(-1)^{\ell(w)-\ell_{P}(w)} \sigma\left(\iota_{P}\left(T_{w}^{P}\right)\right)=(-1)^{\ell(w)-\ell_{P}(w)} \sigma\left(T_{w}^{P}\right)$. We get the lemma.

By Lemma 4.2, we have $\left(\sigma^{\iota_{P}}\right)_{\ell-\ell_{P}}=\left(\sigma_{\ell-\ell_{P}}\right)^{\iota_{P}}$. We denote it by $\sigma_{\ell-\ell_{P}}^{\iota_{P}}$.

Lemma 4.3. We have $\left(\sigma_{\ell-\ell_{P}}\right)_{\ell-\ell_{P}}=\sigma$ and $\left(\sigma^{\iota_{P}}\right)^{\iota_{P}}=\sigma$.

Proof. Obvious from the definition and $\iota_{P}^{2}=$ id.

Lemma 4.4. We have $\left(\sigma_{\ell-\ell_{P}}^{\iota_{P}}\right)_{\ell-\ell_{P}}^{\iota_{P}}=\sigma$.

Proof. The lemma follows from the above lemma and Lemma 4.2 .

Lemma 4.5. Let $\sigma$ be an $\mathcal{H}_{P}$-module and $w \in W_{P}(1)$. 
(1) We have $\sigma_{\ell-\ell_{P}}\left(T_{w}^{P *}\right)=(-1)^{\ell(w)-\ell_{P}(w)} \sigma\left(T_{w}^{P *}\right)$.

(2) For any orientation $o, \sigma_{\ell-\ell_{P}}\left(E_{o}^{P}(w)\right)=(-1)^{\ell(w)-\ell_{P}(w)} \sigma\left(E_{o}^{P}(w)\right)$.

Proof. By Lemma 4.2, we have

$$
\begin{aligned}
\sigma_{\ell-\ell_{P}}\left(T_{w}^{P *}\right) & =(-1)^{\ell_{P}(w)}\left(\sigma_{\ell-\ell_{P}}\right)^{\iota_{P}}\left(T_{w}^{P}\right) \\
& =(-1)^{\ell_{P}(w)}\left(\sigma^{\iota_{P}}\right)_{\ell-\ell_{P}}\left(T_{w}^{P}\right) \\
& =(-1)^{\ell(w)-\ell_{P}(w)}(-1)^{\ell_{P}(w)} \sigma^{\iota_{P}}\left(T_{w}^{P}\right) \\
& =(-1)^{\ell(w)-\ell_{P}(w)} \sigma\left(T_{w}^{P *}\right) .
\end{aligned}
$$

Now we prove (2) by induction on the length of $w$. If $\ell_{P}(w)=0$, then $E_{o}^{P}(w)=T_{w}^{P}$. Hence the lemma follows from the definition of $\sigma_{\ell-\ell_{P}}$.

Assume that $\ell_{P}(w)>0$ and take $s \in S_{\text {aff, } P}(1)$ such that $\ell_{P}\left(s^{-1} w\right)<$ $\ell_{P}(w)$. We have $E_{o}^{P}(w)=E_{o}^{P}(s) E_{o \cdot s}^{P}\left(s^{-1} w\right)$. Since $E_{o}^{P}(s)$ is $T_{s}^{P}$ or $T_{s}^{P *}$, we have $\sigma_{\ell-\ell_{P}}\left(E_{o}^{P}(s)\right)=(-1)^{\ell(s)-\ell_{P}(s)} \sigma\left(E_{o}^{P}(s)\right)$ as we have already proved. By inductive hypothesis,

$$
\begin{aligned}
\sigma_{\ell-\ell_{P}}\left(E_{o}^{P}(w)\right) & =\sigma_{\ell-\ell_{P}}\left(E_{o}^{P}(s)\right) \sigma_{\ell-\ell_{P}}\left(E_{o \cdot s}^{P}\left(s^{-1} w\right)\right. \\
& =(-1)^{\ell(s)-\ell_{P}(s)} \sigma\left(E_{o}^{P}(s)\right)(-1)^{\ell\left(s^{-1} w\right)-\ell_{P}\left(s^{-1} w\right)} \sigma\left(E_{o \cdot s}^{P}\left(s^{-1} w\right)\right) .
\end{aligned}
$$

Since $(-1)^{\ell(s)-\ell_{P}(s)}(-1)^{\ell\left(s^{-1} w\right)-\ell_{P}\left(s^{-1} w\right)}=(-1)^{\ell(w)-\ell_{P}(w)}$, we have

$$
\begin{aligned}
\sigma_{\ell-\ell_{P}}\left(E_{o}^{P}(w)\right) & =(-1)^{\ell(w)-\ell_{P}(w)} \sigma\left(E_{o}^{P}(s)\right) \sigma\left(E_{o \cdot s}^{P}\left(s^{-1} w\right)\right) \\
& =(-1)^{\ell(w)-\ell_{P}(w)} \sigma\left(E_{o}^{P}(s) E_{o \cdot s}^{P}\left(s^{-1} w\right)\right) \\
& =(-1)^{\ell(w)-\ell_{P}(w)} \sigma\left(E_{o}^{P}(w)\right) .
\end{aligned}
$$

We get the lemma.

Remark 4.6. Applying Lemma 4.1 to the right regular representation of $\mathcal{H}_{P}$ (namely, $\pi: \mathcal{H}_{P} \rightarrow \operatorname{End}\left(\mathcal{H}_{P}\right)^{\text {op }}$ defined by $\left.Y \pi(X)=Y X\right)$, we get the following: the linear map $\mathcal{H}_{P} \rightarrow \mathcal{H}_{P}$ defined by $T_{w}^{P} \mapsto(-1)^{\ell(w)-\ell_{P}(w)} T_{w}^{P}$ is an algebra homomorphism. By Lemma 4.5, this map sends $T_{w}^{P *}$ and $E_{o}^{P}(w)$ to $(-1)^{\ell(w)-\ell_{P}(w)} T_{w}^{P *}$ and $(-1)^{\ell(w)-\ell_{P}(w)} E_{o}^{P}(w)$, respectively where $o$ is any orientation.

Lemma 4.7. The algebra homomorphism defined in the remark is identity on $\mathcal{H}_{\mathrm{aff}, P}$. Hence $\left.\sigma_{\ell-\ell_{P}}\right|_{\mathcal{H}_{\mathrm{aff}, P}}=\left.\sigma\right|_{\mathcal{H}_{\mathrm{aff}, P}}$ for any $\mathcal{H}_{P}$-module $\sigma$.

Proof. The algebra $\mathcal{H}_{\text {aff, } P}$ is generated by $T_{s}$ where $s \in S_{\text {aff, } P}(1)$. By Lemma 4.1, $(-1)^{\ell(s)}=-1$. Since the image of $s$ in $W_{P}$ is an affine simple reflection, we have $\ell_{P}(s)=1$. Hence $(-1)^{\ell_{P}(s)}=-1$.

Lemma 4.8. We have $\Delta\left(\sigma_{\ell-\ell_{P}}^{\iota_{P}}\right)=\Delta(\sigma)$.

Proof. Let $\alpha \in \Delta(\sigma) \backslash \Delta_{P}, P_{\alpha}$ a parabolic subgroup corresponding to $\{\alpha\}$ and $\lambda \in \Lambda(1) \cap W_{\text {aff, } P_{\alpha}}(1)$ and we prove $\sigma_{\ell-\ell_{P}}^{\iota_{P}}\left(T_{\lambda}^{P}\right)=1$. Since $\ell_{P}(\lambda)=0$ by Lemma 2.12, we have $\iota_{P}\left(T_{\lambda}^{P}\right)=(-1)^{\ell_{P}(\lambda)} T_{\lambda}^{P *}=T_{\lambda}^{P}$. Hence $\sigma_{\ell-\ell_{P}}\left(T_{\lambda}^{P}\right)=$ $(-1)^{\ell(\lambda)-\ell_{P}(\lambda)} \sigma\left(T_{\lambda}^{P}\right)$. We have $\ell_{P}(\lambda)=0$. Since $\lambda \in \Lambda(1) \cap W_{\text {aff }}(1), \ell(\lambda)$ is even by Lemma 2.14. Hence $\sigma_{\ell-\ell_{P}}^{\iota_{P}}\left(T_{\lambda}^{P}\right)=\sigma\left(T_{\lambda}^{P}\right)=1$. Therefore $\Delta(\sigma) \subset$ $\Delta\left(\sigma_{\ell-\ell_{P}}^{\iota_{P}}\right)$. Applying this to $\sigma_{\ell-\ell_{P}}^{\iota_{P}}$, by Lemma 4.4, we have $\Delta\left(\sigma_{\ell-\ell_{P}}^{\iota_{P}}\right) \subset$ $\Delta(\sigma)$. 
Lemma 4.9. Let $P_{1} \supset P$ be parabolic subgroups of $G$ and $\sigma$ an $\mathcal{H}_{P}$-module

(1) We have $I_{P}^{P_{1}}\left(\sigma_{\ell_{P_{1}}-\ell_{P}}\right)_{\ell-\ell_{P_{1}}} \simeq I_{P}^{P_{1}}\left(\sigma_{\ell-\ell_{P}}\right)$.

(2) For parabolic subgroups $Q \subset Q_{1}$ between $P$ and $P(\sigma)$, we have $\operatorname{St}_{Q}^{Q_{1}}\left(\sigma_{\ell_{Q_{1}}-\ell_{P}}\right)_{\ell-\ell_{Q_{1}}} \simeq \operatorname{St}_{Q}^{Q_{1}}\left(\sigma_{\ell-\ell_{P}}\right)$.

Proof. (1) Define a linear map $f: \mathcal{H}_{P_{1}} \rightarrow \mathcal{H}_{P_{1}}$ by $T_{w}^{P_{1}} \mapsto(-1)^{\ell(w)-\ell_{P_{1}}(w)} T_{w}^{P_{1}}$. Then by Remark 4.6, $f$ is an algebra homomorphism. Put $\varphi^{f}=\varphi \circ f$ for $\varphi \in I_{P}^{P_{1}}\left(\sigma_{\ell_{P_{1}}-\ell_{P}}\right)_{\ell-\ell_{P_{1}}}$. Then for $X \in \mathcal{H}_{P_{1}}$ and $w \in W_{P}^{P_{1}-}(1)$, we have

$$
\begin{aligned}
\varphi^{f}\left(X j_{P}^{P_{1}-*}\left(T_{w}^{P *}\right)\right) & =\varphi^{f}\left(X T_{w}^{P^{1 *}}\right) \\
& =\varphi\left(f(X) f\left(T_{w}^{P_{1} *}\right)\right) \\
& =(-1)^{\ell(w)-\ell_{P_{1}}(w)} \varphi\left(f(X) T_{w}^{P_{1} *}\right) \\
& =(-1)^{\ell(w)-\ell_{P_{1}}(w)} \varphi(f(X)) \sigma_{\ell_{P_{1}}-\ell_{P}}\left(T_{w}^{P *}\right) \\
& =(-1)^{\ell(w)-\ell_{P}(w)} \varphi(f(X)) \sigma\left(T_{w}^{P *}\right) \\
& =\varphi(f(X)) \sigma_{\ell-\ell_{P}}\left(T_{w}^{P *}\right) \\
& =\varphi^{f}(X) \sigma_{\ell-\ell_{P}}\left(T_{w}^{P *}\right) .
\end{aligned}
$$

Hence $\varphi^{f} \in I_{P}^{P_{1}}\left(\sigma_{\ell-\ell_{P}}\right)$. For $w \in W_{P_{1}}(1)$ and $X \in \mathcal{H}_{P_{1}}$, we have

$$
\begin{aligned}
\left(\varphi^{f} T_{w}^{P_{1}}\right)(X) & =\varphi^{f}\left(T_{w}^{P_{1}} X\right) \\
& =\varphi\left(f\left(T_{w}^{P_{1}}\right) f(X)\right) \\
& =(-1)^{\ell(w)-\ell_{P_{1}}(w)} \varphi\left(T_{w}^{P_{1}} f(X)\right) \\
& =\left(\varphi T_{w}^{P_{1}}\right)(f(X))=\left(\varphi T_{w}^{P_{1}}\right)^{f}(X) .
\end{aligned}
$$

Hence $\varphi \mapsto \varphi^{f}$ is an $\mathcal{H}$-module homomorphism, therefore an isomorphism $I_{P}^{P_{1}}\left(\sigma_{\ell_{P_{1}}-\ell_{P}}\right)_{\ell-\ell_{P_{1}}} \rightarrow I_{P}^{P_{1}}\left(\sigma_{\ell-\ell_{P}}\right)$.

(2) First we prove the lemma for $Q=Q_{1}$, namely $\mathrm{St}_{Q}^{Q_{1}}=e_{Q}$. Let $w \in W_{P}(1)$. Then we have

$$
\begin{aligned}
e_{Q}\left(\sigma_{\ell_{Q}-\ell_{P}}\right)_{\ell-\ell_{Q}}\left(T_{w}^{Q *}\right) & =(-1)^{\ell(w)-\ell_{Q}(w)} e_{Q}\left(\sigma_{\ell_{Q}-\ell_{P}}\right)\left(T_{w}^{Q *}\right) \\
& =(-1)^{\ell(w)-\ell_{Q}(w)} \sigma_{\ell_{Q}-\ell_{P}}\left(T_{w}^{P *}\right) \\
& =(-1)^{\ell(w)-\ell_{Q}(w)}(-1)^{\ell_{Q}(w)-\ell_{P}(w)} \sigma\left(T_{w}^{P *}\right) \\
& =(-1)^{\ell(w)-\ell_{P}(w)} \sigma\left(T_{w}^{P *}\right)=\sigma_{\ell-\ell_{P}}\left(T_{w}^{P *}\right) .
\end{aligned}
$$

If $w \in W_{Q \cap P_{2} \text {,aff }}(1)$ then $T_{w}^{Q *} \in \mathcal{H}_{\mathrm{aff}, Q}$. Hence by Lemma 4.7, we have $e_{Q}\left(\sigma_{\ell_{Q}-\ell_{P}}\right)_{\ell-\ell_{Q}}\left(T_{w}^{Q *}\right)=e_{Q}\left(\sigma_{\ell_{Q}-\ell_{P}}\right)\left(T_{w}^{Q *}\right)$. The definition of the extension says that it is 1 . Therefore by a characterization of $e_{Q}\left(\sigma_{\ell-\ell_{P}}\right)$, we have (2) in this case.

In general, consider the exact sequence

$$
\bigoplus_{Q_{1} \supset Q_{2} \supsetneq Q} I_{Q_{2}}^{Q_{1}}\left(e_{Q_{2}}\left(\sigma_{\ell_{Q_{1}}-\ell_{P}}\right)\right) \rightarrow I_{Q}^{Q_{1}}\left(e_{Q}\left(\sigma_{\ell_{Q_{1}}-\ell_{P}}\right)\right) \rightarrow \operatorname{St}_{Q}^{Q_{1}}\left(\sigma_{\ell_{Q_{1}}-\ell_{P}}\right) \rightarrow 0 .
$$


Hence

$$
\begin{aligned}
\bigoplus_{Q_{1} \supset Q_{2} \supsetneq Q} I_{Q_{2}}^{Q_{1}}\left(e_{Q_{2}}\left(\sigma_{\ell_{Q_{1}}-\ell_{P}}\right)\right)_{\ell-\ell_{Q_{1}}} \rightarrow I_{Q}^{Q_{1}}\left(e_{Q}\left(\sigma_{\ell_{Q_{1}}-\ell_{P}}\right)\right)_{\ell-\ell_{Q_{1}}} \\
\rightarrow \mathrm{St}_{Q}^{Q_{1}}\left(\sigma_{\ell_{Q_{1}}-\ell_{P}}\right)_{\ell-\ell_{Q_{1}}} \rightarrow 0 .
\end{aligned}
$$

Using (1) and (2) for $Q=Q_{1}$, for $Q_{1} \supset Q_{2} \supset Q$, we have

$$
\begin{aligned}
I_{Q_{2}}^{Q_{1}}\left(e_{Q_{2}}\left(\sigma_{\ell_{Q_{1}}-\ell_{P}}\right)\right)_{\ell-\ell_{Q_{1}}} & =I_{Q_{2}}^{Q_{1}}\left(e_{Q_{2}}\left(\sigma_{\ell_{Q_{2}}-\ell_{P}}\right)_{\ell_{Q_{1}}-\ell_{Q_{2}}}\right)_{\ell-\ell_{Q_{1}}} \\
& =I_{Q_{2}}^{Q_{1}}\left(e_{Q_{2}}\left(\sigma_{\ell_{Q_{2}}}-\ell_{P}\right) \ell-\ell_{Q_{2}}\right) \\
& =I_{Q_{2}}^{Q_{1}}\left(e_{Q_{2}}\left(\sigma_{\ell-\ell_{P}}\right)\right) .
\end{aligned}
$$

Therefore

$$
\bigoplus_{Q_{1} \supset Q_{2} \supsetneq Q} I_{Q_{2}}^{Q_{1}}\left(e_{Q_{2}}\left(\sigma_{\ell-\ell_{P}}\right)\right) \rightarrow I_{Q}^{Q_{1}}\left(e_{Q}\left(\sigma_{\ell-\ell_{P}}\right)\right) \rightarrow \operatorname{St}_{Q}^{Q_{1}}\left(\sigma_{\ell_{Q_{1}}-\ell_{P}}\right)_{\ell-\ell_{Q_{1}}} \rightarrow 0 .
$$

Hence we get the lemma.

4.2. The functor $I_{P}^{\prime}$. We define the functor $I_{P}^{\prime}$ as follows.

Definition 4.10. For an $\mathcal{H}_{P}$-module $\sigma$, put

$$
I_{P}^{\prime}(\sigma)=\operatorname{Hom}_{\left(\mathcal{H}_{P}^{-}, j_{P}^{-}\right)}(\mathcal{H}, \sigma) .
$$

We remark the following proposition.

Proposition 4.11. Let $P$ be a parabolic subgroup and $\sigma$ an $\mathcal{H}_{P}$-module. Then the map $\varphi \mapsto \varphi \circ \iota$ induces an isomorphism $I_{P}(\sigma)^{\iota} \simeq I_{P}^{\prime}\left(\sigma_{\ell-\ell_{P}}^{\iota_{P}}\right)$.

Proof. For $w \in W_{P}^{-}(1)$, we have

$$
\begin{aligned}
(\varphi \circ \iota)\left(X j_{P}^{-}\left(T_{w}^{P}\right)\right) & =(\varphi \circ \iota)\left(X T_{w}\right) \\
& =\varphi\left(\iota(X) \iota\left(T_{w}\right)\right) \\
& =(-1)^{\ell(w)} \varphi\left(\iota(X) T_{w}^{*}\right) \\
& =(-1)^{\ell(w)} \varphi(\iota(X)) \sigma\left(T_{w}^{P *}\right) \\
& =(-1)^{\ell(w)-\ell_{P}(w)} \varphi^{\iota}(X) \sigma^{\iota P}\left(T_{w}^{P}\right) .
\end{aligned}
$$

Hence $\varphi \circ \iota \in \operatorname{Hom}_{\left(\mathcal{H}_{P}^{-}, j_{P}^{-}\right)}\left(\mathcal{H}, \sigma_{\ell-\ell_{P}}^{\iota_{P}}\right)$. By the same argument implies that $\psi \mapsto \psi \circ \iota$ gives a homomorphism $I_{P}^{\prime}\left(\sigma_{\ell-\ell_{P}}^{\iota_{P}}\right) \rightarrow I_{P}(\sigma)^{\iota}$ which is the inverse of the above homomorphism.

From the properties of $I_{P}$, we get the properties of $I_{P}^{\prime}$.

Proposition 4.12. We have the following.

(1) The functor $I_{P}^{\prime}$ is exact.

(2) The map $\varphi \mapsto\left(\varphi\left(T_{n_{w}}^{*}\right)\right)$ gives an isomorphism $I_{P}^{\prime}(\sigma) \simeq \bigoplus_{w \in W_{0}^{P}} \sigma$.

(3) Let $Q$ be a parabolic subgroup containing $P$. Then $I_{Q}^{\prime} \circ I_{P}^{Q \prime} \simeq I_{P}^{\prime}$ by the homomorphism $\varphi \mapsto(X \mapsto \varphi(X)(1))$.

Proof. (1) follows from the exactness of $I_{P}$. Proposition 2.9 implies (2) and Proposition 2.10 implies (3). 
4.3. Other inductions. The reason why we introduce only one induction $I_{P}^{\prime}$ is that the other two inductions are not new by the following proposition.

Proposition 4.13. Put $P^{\prime}=n_{w_{G} w_{P}} P^{\mathrm{op}} n_{w_{G} w_{P}}^{-1}$.

(1) The map $\varphi \mapsto\left(X \mapsto \varphi\left(X T_{n_{w_{G} w_{P}}}\right)\right)$ gives an isomorphism

$$
I_{P}^{\prime}(\sigma)=\operatorname{Hom}_{\left(\mathcal{H}_{P}^{-}, j_{P}^{-}\right)}(\mathcal{H}, \sigma) \stackrel{\sim}{\rightarrow} \operatorname{Hom}_{\left(\mathcal{H}_{P^{\prime}}^{+}, j_{P^{\prime}}^{+}\right)}\left(\mathcal{H}, n_{w_{G} w_{P}} \sigma\right) .
$$

(2) The map $\varphi \mapsto\left(X \mapsto \varphi\left(X T_{n_{w_{G} w_{P}}^{*}}^{*}\right)\right)$ gives an isomorphism

$$
I_{P}(\sigma)=\operatorname{Hom}_{\left(\mathcal{H}_{P}^{-}, j_{P}^{-*}\right)}(\mathcal{H}, \sigma) \stackrel{\sim}{\rightarrow} \operatorname{Hom}_{\left(\mathcal{H}_{P^{\prime}}^{+}, j_{P^{\prime}}^{+*}\right)}\left(\mathcal{H}, n_{w_{G} w_{P}} \sigma\right) .
$$

Remark 4.14. By $\varphi \mapsto \varphi \circ \iota$, we have

$$
\operatorname{Hom}_{\left(\mathcal{H}_{P}^{+}, j_{P}^{+}\right)}(\mathcal{H}, \sigma)^{\iota} \simeq \operatorname{Hom}_{\left(\mathcal{H}_{P}^{+}, j_{P}^{+*}\right)}\left(\mathcal{H}, \sigma_{\ell-\ell_{P}}^{\iota_{P}}\right) .
$$

Hence the statement (2) follows from (1).

First we check that the map is a homomorphism. For the calculation, we need the following lemma. Recall the notation ${ }^{P} W_{0}$ from subsection 2.9.

Lemma 4.15. Let $w \in{ }^{P} W_{0}, v \in W_{0, P}$ and $\lambda \in Z\left(W_{P}(1)\right) Z_{\kappa}$. For $o=o_{+}$ or $o_{-}$, we have $E_{o \cdot v}\left(\lambda n_{w}\right)=E_{o}\left(\lambda n_{w}\right)$.

Proof. We may assume $\lambda \in Z\left(W_{P}(1)\right)$. We prove the lemma in $\mathcal{H}\left[q_{s}^{ \pm 1}\right]$. First we assume $o=o_{-}$and prove the lemma by induction on $\ell(w)$. Assume that $w=1$. Take anti-dominant $\lambda_{1}, \lambda_{2} \in Z\left(W_{P}(1)\right)$ such that $\lambda=\lambda_{1} \lambda_{2}^{-1}$. Then by (2.1), we have $E_{o_{-}}(\lambda) E_{o_{-}}\left(\lambda_{2}\right)=q_{\lambda}^{1 / 2} q_{\lambda_{2}}^{1 / 2} q_{\lambda_{1}}^{-1 / 2} E_{o_{-}}\left(\lambda_{1}\right)$. Since $\lambda_{1}, \lambda_{2}$ are anti-dominant, we have $E_{o_{-}}\left(\lambda_{1}\right)=T_{\lambda_{1}}$ and $E_{o_{-}}\left(\lambda_{2}\right)=T_{\lambda_{2}}$ by (2.3). Hence $E_{o_{-}}(\lambda)=q_{\lambda}^{1 / 2} q_{\lambda_{1}}^{-1 / 2} q_{\lambda_{2}}^{1 / 2} T_{\lambda_{1}} T_{\lambda_{2}}^{-1}$. Since $n_{v}^{-1} \cdot \lambda_{1}=\lambda_{1}$ and $n_{v}^{-1} \cdot \lambda_{2}=\lambda_{2}$ are both anti-dominant, we have $E_{o_{-} \cdot v}(\lambda)=q_{\lambda}^{1 / 2} q_{\lambda_{1}}^{-1 / 2} q_{\lambda_{2}}^{1 / 2} T_{\lambda_{1}} T_{\lambda_{2}}^{-1}$ by the same argument. Hence we get the lemma in this case.

Assume that $\ell(w)>0$ and take $s \in S_{0}$ such that $w s<w$. Then by [Deo77, Lemma 3.1], we have $w s \in{ }^{P} W_{0}$. By the product formula (2.1), we have

$$
\begin{aligned}
E_{o_{-}}\left(\lambda n_{w}\right) & =q_{\lambda n_{w}}^{1 / 2} q_{\lambda n_{w s}}^{-1 / 2} q_{n_{s}}^{-1 / 2} E_{o_{-}}\left(\lambda n_{w s}\right) E_{o_{-} \cdot w s}\left(n_{s}\right), \\
E_{o_{-} \cdot v}\left(\lambda n_{w}\right) & =q_{\lambda n_{w}}^{1 / 2} q_{\lambda n_{w s}}^{-1 / 2} q_{n_{s}}^{-1 / 2} E_{o_{-} \cdot v}\left(\lambda n_{w s}\right) E_{o_{-} \cdot v w s}\left(n_{s}\right) .
\end{aligned}
$$

By inductive hypothesis, we have $E_{o_{-}}\left(\lambda n_{w s}\right)=E_{o_{-} \cdot v}\left(\lambda n_{w s}\right)$. Hence it is sufficient to prove that $E_{o_{-} \cdot w s}\left(n_{s}\right)=E_{o_{-} \cdot v w s}\left(n_{s}\right)$. Since $w s<w$, we have $E_{o_{-} \cdot w s}\left(n_{s}\right)=T_{n_{s}}$ by (2.4). Take $\alpha \in \Delta$ such that $s=s_{\alpha}$. Then $w s(\alpha)=$ $-w(\alpha)>0$. If vws $(\alpha)<0$, then $w s(\alpha) \in \Sigma_{P}^{+}$since $v \in W_{0, P}$. Since $w \in$ ${ }^{P} W_{0}$, we have $-\alpha=w^{-1}(w s(\alpha)) \in w^{-1}\left(\Sigma_{P}^{+}\right) \subset \Sigma^{+}$. This is a contradiction. Hence $v w s(\alpha)>0$. Therefore vws $<v w$. By (2.4), we have $E_{o_{-} \cdot v w s}\left(n_{s}\right)=$ $T_{n_{s}}$. We get $E_{o_{-} \cdot w s}\left(n_{s}\right)=E_{o_{-} \cdot v w s}\left(n_{s}\right)$ and finish the inductive step.

Now we get $E_{O_{-} \cdot v}\left(\lambda n_{w}\right)=E_{O_{-}}\left(\lambda n_{w}\right)$. Applying $\iota$ to both sides with [Vig16, Lemma 5.31], we get $(-1)^{\ell\left(\lambda n_{w}\right)} E_{o_{+} \cdot v}\left(\lambda n_{w}\right)=(-1)^{\ell\left(\lambda n_{w}\right)} E_{o_{+}}\left(\lambda n_{w}\right)$. Hence $E_{o_{+} \cdot v}\left(\lambda n_{w}\right)=E_{o_{+}}\left(\lambda n_{w}\right)$.

We start to prove Proposition 4.13.

Lemma 4.16. The map given in Proposition 4.13 is an $\mathcal{H}$-module homomorphism. 
Proof. Put $n=n_{w_{G} w_{P}}$. The lemma is equivalent to that the map $\varphi \mapsto$ $\varphi\left(T_{n}\right)$ from $I_{P}^{\prime}(\sigma)$ to $n \sigma$ gives an $\left(\mathcal{H}_{P^{\prime}}^{+}, j_{P^{\prime}}^{+}\right)$-module homomorphism. Let $w \in W_{P^{\prime}}(1)$ be a $P^{\prime}$-positive element. We have

$$
\left(\varphi j_{P^{\prime}}^{+}\left(E_{o_{+, P^{\prime}}}^{P^{\prime}}(w)\right)\right)\left(T_{n}\right)=\varphi\left(j_{P^{\prime}}^{+}\left(E_{o_{+, P^{\prime}}}^{P^{\prime}}(w)\right) T_{n}\right) .
$$

and, by Lemma 2.6, we have

$$
j_{P^{\prime}}^{+}\left(E_{o_{+, P^{\prime}}}^{P^{\prime}}(w)\right)=j_{P^{\prime}}^{+}\left(E_{o_{-, P^{\prime}} \cdot w_{P^{\prime}}}^{P^{\prime}}(w)\right)=E_{o_{-} \cdot w_{P^{\prime}}}(w) .
$$

We have $w_{G} w_{P}=w_{P^{\prime}} w_{G} \in P^{\prime} W_{0}$. Hence by Lemma 4.15, we have $T_{n}=$ $E_{o_{-}}(n)=E_{o_{-} \cdot n_{w_{P^{\prime}}} w}(n)$. By Lemma 2.18, we have $\ell(w)+\ell(n)=\ell(w n)=$ $\ell\left(n n^{-1} w n\right)=\ell(n)+\ell\left(n^{-1} w n\right)$. Here we use that $n \in W_{0}^{P}$ and $n^{-1} w n \in$ $W_{P}(1)$ is $P$-negative. Therefore

$$
\begin{aligned}
E_{o_{-} \cdot w_{P^{\prime}}}(w) T_{n} & =E_{o_{-} \cdot w_{P^{\prime}}}(w) E_{o_{-} \cdot n_{w_{P^{\prime}}} w}(n) \\
& =E_{o_{-} \cdot w_{P^{\prime}}}(w n) \\
& =E_{o_{-} \cdot w_{P^{\prime}}}(n) E_{o_{-} \cdot n_{w_{P^{\prime}}} n}\left(n^{-1} w n\right) \\
& =T_{n} E_{o_{-} \cdot n_{w_{P^{\prime}}}}\left(n^{-1} w n\right) .
\end{aligned}
$$

Since $n_{w_{P^{\prime}}} n=n_{w_{P^{\prime}}} n_{w_{G} w_{P}}=n_{w_{G}}$, we have $o_{-} \cdot n_{w_{P}} n=o_{-} \cdot w_{G}=o_{+}$. Hence $E_{o_{-} \cdot n_{w_{P^{\prime}}} n}\left(n^{-1} w n\right)=E_{o_{+}}\left(n^{-1} w n\right)=j_{P}^{-}\left(E_{o_{+}, P}^{P}\left(n^{-1} w n\right)\right)$ by Lemma 2.6. Therefore we have

$$
j_{P^{\prime}}^{+}\left(E_{o_{+, P^{\prime}}}^{P^{\prime}}(w)\right) T_{n}=T_{n} j_{P}^{-}\left(E_{o_{+, P}}^{P}\left(n^{-1} w n\right)\right) .
$$

Hence we get

$$
\begin{aligned}
\varphi\left(j_{P^{\prime}}^{+}\left(E_{o_{+, P^{\prime}}}^{P^{\prime}}(w)\right) T_{n}\right) & =\varphi\left(T_{n} j_{P}^{-}\left(E_{o_{+, P}}^{P}\left(n^{-1} w n\right)\right)\right) \\
& =\varphi\left(T_{n}\right) \sigma\left(E_{o_{+, P}}^{P}\left(n^{-1} w n\right)\right) \\
& =\varphi\left(T_{n}\right)(n \sigma)\left(E_{o_{+, P^{\prime}}}^{P^{\prime}}(w)\right) .
\end{aligned}
$$

We get the lemma.

We construct the homomorphism in the opposite direction.

Lemma 4.17. Let $\lambda=\lambda_{P}^{-} \in Z\left(W_{P}(1)\right)$ as in Proposition 2.5. Put $n=$ $n_{w_{G} w_{P}}$ and $P^{\prime}=n P^{\mathrm{op}} n^{-1}$. Then $\varphi \mapsto\left(X \mapsto \varphi\left(X E_{o_{+}}\left(\lambda n^{-1}\right)\right)\right)$ gives a homomorphism

$$
\operatorname{Hom}_{\left(\mathcal{H}_{P^{\prime}}^{+}, j_{P^{\prime}}^{+}\right)}(\mathcal{H}, n \sigma) \rightarrow \operatorname{Hom}_{\left(\mathcal{H}_{P}^{-}, j_{P}^{-}\right)}(\mathcal{H}, \sigma)=I_{P}^{\prime}(\sigma) .
$$

Proof. We prove that $\varphi \mapsto \varphi\left(E_{O_{+}}\left(\lambda n^{-1}\right)\right)$ is an $\left(\mathcal{H}_{P}^{-}, j_{P}^{-}\right)$-homomorphism $\operatorname{Hom}_{\left(\mathcal{H}_{P^{\prime}}^{+}, j_{P}^{+}\right)}(\mathcal{H}, n \sigma) \rightarrow \sigma$. Let $w \in W_{P}(1)$ be a $P$-negative element. Then

$$
\left(\varphi j_{P}^{-}\left(E_{o_{+}, P}^{P}(w)\right)\right)\left(E_{o_{+}}\left(\lambda n^{-1}\right)\right)=\varphi\left(j_{P}^{-}\left(E_{o_{+, P}}^{P}(w)\right) E_{o_{+}}\left(\lambda n^{-1}\right)\right)
$$

and $j_{P}^{-}\left(E_{o_{+}, P}^{P}(w)\right)=E_{o_{+}}(w)$ by Lemma 2.6. Since $w \in W_{P}(1), \lambda \in$ $Z\left(W_{P}(1)\right)$ and $\left(w_{G} w_{P}\right)^{-1} \in{ }^{P} W_{0}$, we have $E_{o_{+}}\left(\lambda n^{-1}\right)=E_{o_{+} \cdot w}\left(\lambda n^{-1}\right)$ by Lemma 4.15]. We also have, by Lemma 2.19, $\ell(w)+\ell\left(\lambda n^{-1}\right)=\ell\left(w \lambda n^{-1}\right)$. Hence

$$
E_{o_{+}}(w) E_{o_{+}}\left(\lambda n^{-1}\right)=E_{o_{+}}(w) E_{o_{+} \cdot w}\left(\lambda n^{-1}\right)=E_{o_{+}}\left(w \lambda n^{-1}\right) .
$$


Since $\lambda \in Z\left(W_{P}(1)\right)$, we have $w \lambda n^{-1}=\lambda w n^{-1}=\lambda n^{-1}\left(n w n^{-1}\right)$. The element $n w n^{-1}$ is $P^{\prime}$-positive. By Lemma 2.19, $\ell\left(\lambda n^{-1} n w n^{-1}\right)=\ell\left(\lambda n^{-1}\right)+$ $\ell\left(n w n^{-1}\right)$. (We have $\lambda n^{-1}=n^{-1}\left(n \lambda n^{-1}\right)$ and since $n\left(\Sigma^{+} \backslash \Sigma_{P}^{+}\right)=\Sigma^{-} \backslash$ $\Sigma_{P^{\prime}}^{-}, n \lambda n^{-1}$ satisfies the condition of $\lambda_{P^{\prime}}^{+}$in Proposition [2.5. We also have $\left(w_{G} w_{P}\right)^{-1}=w_{P} w_{G}=w_{G} w_{P^{\prime}} \in W_{0}^{P^{\prime}}$.) Hence we have $E_{o_{+}}\left(w \lambda n^{-1}\right)=$ $E_{o_{+}}\left(\lambda n^{-1}\right) E_{o_{+} \cdot n^{-1}}\left(n w n^{-1}\right)$. We have $o_{+} \cdot n^{-1}=o_{+} \cdot w_{P} w_{G}=o_{+} \cdot w_{G} w_{P^{\prime}}=$ $o_{-} \cdot w_{P^{\prime}}$. Hence we have

$$
\begin{aligned}
E_{o_{+} \cdot n^{-1}}\left(n w n^{-1}\right) & =E_{o_{-} \cdot w_{P^{\prime}}}\left(n w n^{-1}\right) \\
& =j_{P^{\prime}}^{+}\left(E_{o_{-, P^{\prime}} \cdot w_{P^{\prime}}}^{P^{\prime}}\left(n w n^{-1}\right)\right) \\
& =j_{P^{\prime}}^{+}\left(E_{o_{+, P^{\prime}}}^{P^{\prime}}\left(n w n^{-1}\right)\right)
\end{aligned}
$$

by Lemma 2.6. Therefore, we have

$$
j_{P}^{-}\left(E_{o_{+}, P}^{P}(w)\right) E_{o_{+}}\left(\lambda n^{-1}\right)=E_{o_{+}}\left(\lambda n^{-1}\right) j_{P^{\prime}}^{+}\left(E_{o_{+, P^{\prime}}}^{P^{\prime}}\left(n w n^{-1}\right)\right)
$$

Therefore

$$
\begin{aligned}
\left(\varphi j_{P}^{-}\left(E_{o_{+}, P}^{P}(w)\right)\right)\left(E_{o_{+}}\left(\lambda n^{-1}\right)\right) & =\varphi\left(j_{P}^{-}\left(E_{o_{+}, P}^{P}(w)\right)\left(E_{o_{+}}\left(\lambda n^{-1}\right)\right)\right) \\
& =\varphi\left(E_{o_{+}}\left(\lambda n^{-1}\right) j_{P^{\prime}}^{+}\left(E_{o_{+, P^{\prime}}}^{P^{\prime}}\left(n w n^{-1}\right)\right)\right) \\
& =\varphi\left(E_{o_{+}}\left(\lambda n^{-1}\right)\right)(n \sigma)\left(E_{o_{+, P^{\prime}}}^{P^{\prime}}\left(n w n^{-1}\right)\right) \\
& =\varphi\left(E_{o_{+}}\left(\lambda n^{-1}\right)\right) \sigma\left(E_{o_{+, P}}^{P}(w)\right) .
\end{aligned}
$$

We get the lemma.

Proof of Proposition 4.13. We prove that the compositions of the homomorphisms in Proposition 4.13 and Lemma 4.17 are isomorphisms. Let $\Phi$ be the homomorphism in Proposition 4.13 and $\Psi$ that in Lemma 4.17.

Put $n=n_{w_{G} w_{P}}$ and $P^{\prime}=n P^{\mathrm{op}} n^{-1}$. For $\varphi \in \operatorname{Hom}_{\left(\mathcal{H}_{P^{\prime}}^{+}, j_{P^{\prime}}^{+}\right)}(\mathcal{H}, n \sigma)$, $\Phi(\Psi(\varphi))$ is given by

$$
\Phi(\Psi(\varphi))(X)=\Psi(\varphi)\left(X T_{n}\right)=\varphi\left(X T_{n} E_{o_{+}}\left(\lambda n^{-1}\right)\right)
$$

where $\lambda=\lambda_{P}^{-}$as in Proposition 2.5. We have $T_{n}=E_{o_{-}}(n)$ by (2.4). Since $w_{G} w_{P}=w_{P^{\prime}} w_{G} \in P^{\prime} W_{0}$, we have $E_{o_{-}}(n)=E_{o_{-} \cdot w_{P^{\prime}}}(n)$ by Lemma 4.15, We have $o_{-} \cdot w_{P^{\prime}} n=o_{-} \cdot w_{G}=o_{+}$. Since $\left(w_{G} w_{P}\right)^{-1}=w_{P} w_{G} \in{ }^{P} W_{0}$, by Lemma 2.19 and 2.15, we have $\ell\left(\lambda n^{-1}\right)=\ell(\lambda)-\ell(n)=\ell(n \cdot \lambda)-\ell(n)$. Hence, by Lemma 2.6, we have

$$
\begin{aligned}
T_{n} E_{o_{+}}\left(\lambda n^{-1}\right) & =E_{o_{-} \cdot w_{P^{\prime}}}(n) E_{o_{-} \cdot w_{P^{\prime}} n}\left(\lambda n^{-1}\right) \\
& =E_{o_{-} \cdot w_{P^{\prime}}}\left(n \lambda n^{-1}\right) \\
& =j_{P^{\prime}}^{+}\left(E_{o_{-, P^{\prime}} \cdot w_{P^{\prime}}}^{P^{\prime}}\left(n \lambda n^{-1}\right)\right) \\
& =j_{P^{\prime}}^{+}\left(E_{o_{+, P^{\prime}}}^{P^{\prime}}\left(n \lambda n^{-1}\right)\right) .
\end{aligned}
$$

Therefore, we have

$$
\begin{aligned}
\Phi(\Psi(\varphi))(X) & =\varphi\left(X j_{P^{\prime}}^{+}\left(E_{o_{+, P^{\prime}}}^{P^{\prime}}\left(n \lambda n^{-1}\right)\right)\right) \\
& =\varphi(X)(n \sigma)\left(E_{o_{+, P^{\prime}}}^{P^{\prime}}\left(n \lambda n^{-1}\right)\right) \\
& =\varphi(X) \sigma\left(E_{o_{+, P}}^{P}(\lambda)\right) .
\end{aligned}
$$


Since $\lambda$ is in $Z\left(W_{P}(1)\right), \sigma\left(E_{o_{+}, P}^{P}(\lambda)\right)$ is invertible. Hence $\Phi \circ \Psi$ is invertible.

Next, for $\psi \in I_{P}^{\prime}(\sigma)=\operatorname{Hom}_{\left(\mathcal{H}_{P}^{-}, j_{P}^{-}\right)}(\mathcal{H}, \sigma)$, we have

$$
\Psi(\Phi(\psi))(X)=\psi\left(X E_{O_{+}}\left(\lambda n^{-1}\right) T_{n}\right) .
$$

As in the above argument, we have $T_{n}=E_{o_{-} \cdot w_{P^{\prime}}}(n)=E_{o_{+} \cdot n^{-1}}(n)$. We also have $\ell(\lambda)=\ell\left(\lambda n^{-1}\right)+\ell(n)$ as in the above. We have

$$
E_{o_{+}}\left(\lambda n^{-1}\right) T_{n}=E_{o_{+}}\left(\lambda n^{-1}\right) E_{o_{+} \cdot n^{-1}}(n)=E_{o_{+}}(\lambda)=j_{P}^{-}\left(E_{o_{+}, P}^{P}(\lambda)\right) .
$$

Therefore we have

$$
\Psi(\Phi(\psi))(X)=\psi\left(X j_{P}^{-}\left(E_{o_{+, P}}^{P}(\lambda)\right)\right)=\psi(X) \sigma\left(E_{o_{+}, P}^{P}(\lambda)\right) .
$$

Since $\lambda$ is in $Z\left(W_{P}(1)\right), \sigma\left(E_{o_{+}, P}^{P}(\lambda)\right)$ is invertible. Hence $\Psi \circ \Phi$ is invertible.

4.4. Tensor products. Recall that we have

$$
I_{P}(\sigma) \simeq n_{w_{G} w_{P}} \sigma \otimes_{\left(\mathcal{H}_{P^{\prime}}^{+}, j_{P^{\prime}}^{+}\right)} \mathcal{H} .
$$

where $P^{\prime}=n_{w_{G} w_{P}} P^{\mathrm{op}} n_{w_{G} w_{P}}^{-1}$ by Proposition 2.21] Again, we can consider the four inductions defined via the tensor product. By $x \otimes X \mapsto x \otimes \iota(X)$, we have

and

$$
\left(\sigma \otimes_{\left(\mathcal{H}_{P}^{+}, j_{P}^{+}\right)} \mathcal{H}\right)^{\iota} \simeq \sigma_{\ell-\ell_{P}}^{\iota_{P}} \otimes_{\left(\mathcal{H}_{P}^{+}, j_{P}^{+*}\right)} \mathcal{H}
$$

$$
\left(\sigma \otimes_{\left(\mathcal{H}_{P}^{-}, j_{P}^{-}\right)} \mathcal{H}\right)^{\iota} \simeq \sigma_{\ell-\ell_{P}}^{\iota_{P}} \otimes_{\left(\mathcal{H}_{P}^{-}, j_{P}^{-*}\right)} \mathcal{H} .
$$

Proposition 4.18. Let $P$ be a parabolic subgroup and $\sigma$ an $\mathcal{H}_{P}$-module. Put $P^{\prime}=n_{w_{G} w_{P}} P^{\mathrm{op}} n_{w_{G} w_{P}}^{-1}$.

(1) The map $x \otimes X \mapsto x \otimes T_{n_{w_{G} w_{P}}} X$ gives an isomorphism

$$
\sigma \otimes_{\left(\mathcal{H}_{P}^{-}, j_{P}^{-}\right)} \mathcal{H} \rightarrow n_{w_{G} w_{P}} \sigma \otimes_{\left(\mathcal{H}_{P^{\prime}}^{+}, j_{P^{\prime}}^{+}\right)} \mathcal{H} .
$$

(2) The map $x \otimes X \mapsto x \otimes T_{n_{w_{G} w_{P}}^{*}}^{*} X$ gives an isomorphism

$$
\sigma \otimes_{\left(\mathcal{H}_{P}^{-}, j_{P}^{-*}\right)} \mathcal{H} \rightarrow n_{w_{G} w_{P}} \sigma \otimes_{\left(\mathcal{H}_{P^{\prime}}^{+}, j_{P^{\prime}}^{+*}\right)} \mathcal{H}
$$

Proof. (2) follows from (1). We prove (1).

Put $n=n_{w_{G} w_{P}}$. Let $\Phi$ be a homomorphism given in the proposition and first we prove that this is a well-defined $\mathcal{H}$-homomorphism. We prove that the linear map $x \mapsto x \otimes T_{n}$ is an $\left(\mathcal{H}_{P}^{-}, j_{P}^{-}\right)$-homomorphism $\sigma \rightarrow n \sigma \otimes_{\left(\mathcal{H}_{P^{\prime}}^{+}, j_{P^{\prime}}^{+}\right)}$ $\mathcal{H}$. Let $x \in \sigma$ and $w \in W_{P}^{-}(1)$. Then $n w n^{-1} \in W_{P^{\prime}}^{+}(1)$. Hence by (4.1), we have

$$
j_{P^{\prime}}^{+}\left(E_{o_{+, P^{\prime}}}^{P^{\prime}}\left(n w n^{-1}\right)\right) T_{n}=T_{n} j_{P}^{-}\left(E_{o_{+}, P}^{P}(w)\right)
$$

Hence, in $n \sigma \otimes_{\left(\mathcal{H}_{P^{\prime}}^{+}, j_{P^{\prime}}^{+}\right)} \mathcal{H}$, we have

$$
\begin{aligned}
x \otimes T_{n} j_{P}^{-}\left(E_{o_{+, P}}^{P}(w)\right) & =x \otimes j_{P^{\prime}}^{+}\left(E_{o_{+, P^{\prime}}}^{P^{\prime}}\left(n w n^{-1}\right)\right) T_{n} \\
& =x(n \sigma)\left(E_{o_{+, P^{\prime}}^{\prime}}^{P^{\prime}}\left(n w n^{-1}\right)\right) \otimes T_{n} \\
& =x \sigma\left(E_{o_{+, P}}^{P}(w)\right) \otimes T_{n} .
\end{aligned}
$$

Therefore $\Phi$ is an $\mathcal{H}$-module homomorphism. 
Next let $\lambda=\lambda_{P}^{-}$and consider the linear map $\Psi: x \otimes X \mapsto x \otimes E_{o_{+}}\left(\lambda n^{-1}\right) X$. We prove that $\Psi$ is also a well-defined $\mathcal{H}$-homomorphism $n \sigma \otimes_{\left(\mathcal{H}_{P^{\prime}}^{+}, j_{P^{\prime}}^{+}\right)} \mathcal{H} \rightarrow$ $\sigma \otimes_{\left(\mathcal{H}_{P}^{-}, j_{P}^{-}\right)} \mathcal{H}$. Let $w \in W_{P^{\prime}}^{+}(1)$ and $x \in \sigma$. Then $n^{-1} w n \in W_{P}^{-}(1)$. By (4.2), we have

$$
j_{P}^{-}\left(E_{o_{+, P}}^{P}\left(n^{-1} w n\right)\right) E_{o_{+}}\left(\lambda n^{-1}\right)=E_{o_{+}}\left(\lambda n^{-1}\right) j_{P^{\prime}}^{+}\left(E_{o_{+, P^{\prime}}}^{P^{\prime}}(w)\right) .
$$

Hence, in $\sigma \otimes_{\left(\mathcal{H}_{P}^{-}, j_{P}^{-}\right)} \mathcal{H}$, we have

$$
\begin{aligned}
x \otimes E_{o_{+}}\left(\lambda n^{-1}\right) j_{P^{\prime}}^{+}\left(E_{o_{+, P^{\prime}}}^{P^{\prime}}(w)\right) & =x \otimes j_{P}^{-}\left(E_{o_{+, P}}^{P}\left(n^{-1} w n\right)\right) E_{o_{+}}\left(\lambda n^{-1}\right) \\
& =x \sigma\left(E_{o_{+, P}}^{P}\left(n^{-1} w n\right)\right) \otimes E_{o_{+}}\left(\lambda n^{-1}\right) \\
& =x(n \sigma)\left(E_{o_{+, P^{\prime}}}^{P^{\prime}}(w)\right) \otimes E_{o_{+}}\left(\lambda n^{-1}\right) .
\end{aligned}
$$

Therefore $\Psi$ is an $\mathcal{H}$-homomorphism.

Let $x \in \sigma$ and $X \in \mathcal{H}$. By (4.3), we have

$$
\begin{aligned}
\Phi(\Psi(x \otimes X)) & =x \otimes T_{n} E_{o_{+}}\left(\lambda n^{-1}\right) X \\
& =x \otimes j_{P^{\prime}}^{+}\left(E_{o_{+, P^{\prime}}}^{P^{\prime}}\left(n \lambda n^{-1}\right)\right) X \\
& =x(n \sigma)\left(E_{o_{+, P^{\prime}}}^{P^{\prime}}\left(n \lambda n^{-1}\right)\right) \otimes X \\
& =x \sigma\left(E_{o_{+}, P}^{P}(\lambda)\right) \otimes X .
\end{aligned}
$$

Since $\lambda \in Z\left(W_{P}(1)\right), \sigma\left(E_{o_{+}, P}^{P}(\lambda)\right)$ is invertible. Hence $\Phi \circ \Psi$ is invertible. By (4.4), we also have

$$
\begin{aligned}
\Psi(\Phi(x \otimes X)) & =x \otimes E_{o_{+}}\left(\lambda n^{-1}\right) T_{n} X \\
& =x \otimes j_{P}^{-}\left(E_{o_{+} P}^{P}(\lambda)\right) X \\
& =x \sigma\left(E_{o_{+, P}}^{P}(\lambda)\right) \otimes X .
\end{aligned}
$$

This is again invertible.

Corollary 4.19. Let $P$ be a parabolic subgroup and $\sigma$ an $\mathcal{H}_{P}$-module. Then we have

$$
\begin{aligned}
& I_{P}(\sigma) \simeq \sigma \otimes_{\left(\mathcal{H}_{P}^{-}, j_{P}^{-}\right)} \mathcal{H}, \\
& I_{P}^{\prime}(\sigma) \simeq \sigma \otimes_{\left(\mathcal{H}_{P}^{-}, j_{P}^{-*}\right)} \mathcal{H} .
\end{aligned}
$$

Proof. The first one follows from the Propositions 2.21 and 4.18 and the second one follows from the twist of the first one.

Proposition 4.20. Let $P$ be a parabolic subgroup and $\sigma$ an $\mathcal{H}_{P}$-module.

(1) The isomorphism $\sigma \otimes_{\left(\mathcal{H}_{P}^{-}, j_{P}^{-}\right)} \mathcal{H} \rightarrow I_{P}(\sigma)$ is given by the following. For $x \in \sigma$, let $\varphi_{x} \in I_{P}(\sigma)$ be an element such that $\varphi_{x}(1)=x$ and $\varphi_{x}\left(T_{n_{w}}^{*}\right)=0$ for any $w \in W_{0}^{P} \backslash\{1\}$. Then the isomorphism is given by $x \otimes X \mapsto \varphi_{x} X$.

(2) The isomorphism $\sigma \otimes_{\left(\mathcal{H}_{P}^{-}, j_{P}^{-*}\right)} \mathcal{H} \rightarrow I_{P}^{\prime}(\sigma)$ is given by the following. For $x \in \sigma$, let $\varphi_{x} \in I_{P}(\sigma)$ be an element such that $\varphi_{x}(1)=x$ and $\varphi_{x}\left(T_{n_{w}}\right)=0$ for any $w \in W_{0}^{P} \backslash\{1\}$. Then the isomorphism is given by $x \otimes X \mapsto \varphi_{x} X$.

In particular, these isomorphisms do not depend on a choice of a lift $n_{w_{G} w_{P}}$. 
Proof. The second statement follows from the first one. From the construction, the image of $x \in \sigma$ under the isomorphism in Corollary 4.19 is given by $\psi_{x} T_{n_{w_{G} w_{P}}}$ where $\psi_{x}$ is characterized by $\psi_{x}\left(T_{n_{w_{G} w_{P}}}\right)=x$ and $\psi_{x}\left(T_{n_{w}}\right)=0$ for any $w \in W_{0}^{P} \backslash\left\{w_{G} w_{P}\right\}$. We prove $\psi_{x} T_{n_{w_{G} w_{P}}}=\varphi_{x}$. then

Set $\psi=\psi_{x} T_{n_{w_{G} w_{P}}}$. We have $\psi(1)=\psi_{x}\left(T_{n_{w_{G} w_{P}}}\right)=x$. If $w \in W_{0}^{P} \backslash\{1\}$,

$$
\begin{aligned}
T_{n_{w_{G} w_{P}}} T_{n_{w}}^{*} & =E_{o_{+} \cdot\left(w_{G} w_{P}\right)^{-1}}\left(n_{w_{G} w_{P}}\right) E_{o_{+}}\left(n_{w}\right) \\
& =q_{w_{G} w_{P}}^{1 / 2} q_{w}^{1 / 2} q_{w_{G} w_{P} w}^{-1 / 2} E_{o_{+} \cdot\left(w_{G} w_{P}\right)^{-1}}\left(n_{w_{G} w_{P}} n_{w}\right) \\
& \in \sum_{v \in W_{0}, v \leq w_{G} w_{P} w} C\left[Z_{\kappa}\right] T_{n_{v}} .
\end{aligned}
$$

Hence it is sufficient to prove that if $v \leq w_{G} w_{P} w$ then $\psi_{x}\left(T_{n_{v}}\right)=0$. Since $w \notin W_{P, 0}$, we have $w_{G} w_{P} w \notin w_{G} w_{P} W_{P, 0}$. Hence by Abe, Lemma 4.13 (3)], we have $v \notin w_{G} w_{P} W_{P, 0}$. Take $v_{1} \in W_{0}^{P}$ and $v_{2} \in W_{P, 0}$ such that $v=v_{1} v_{2}$. Then we have $\psi_{x}\left(T_{n_{v}}\right)=\psi_{x}\left(T_{n_{v_{1}}} T_{n_{v_{2}}}\right)=\psi_{x}\left(T_{n_{v_{1}}}\right) \sigma\left(T_{n_{v_{2}}}^{P}\right)$. Since $v_{1} \neq w_{G} w_{P}$, this is zero.

\section{Adjoint Functors}

5.1. Adjoint functors $L_{P}$ and $R_{P}$. Let $P$ be a parabolic subgroup. By the definition of the parabolic induction $I_{P}$, it has the left adjoint functor $L_{P}$. The functor $L_{P}$ is defined by

$$
L_{P}(\pi)=\pi \otimes_{\left(\mathcal{H}_{P}^{-}, j_{P}^{-*}\right)} \mathcal{H}_{P}=\pi E_{o_{-}}\left(\lambda_{P}^{-}\right)^{-1}
$$

where $\lambda_{P}^{-}$is as in Proposition 2.5. Since this is a localization, this functor is exact.

By Proposition 2.21, the functor $I_{P}$ also has the right adjoint functor $R_{P}$. It is defined as follows. Set $P^{\prime}=n_{w_{G} w_{P}} P^{\mathrm{op}} n_{w_{G} w_{P}}^{-1}$. Then we have

$$
R_{P}(\pi)=n_{w_{G} w_{P}}^{-1} \operatorname{Hom}_{\left(\mathcal{H}_{P^{\prime}}^{+}, j_{P^{\prime}}^{+}\right)}\left(\mathcal{H}_{P^{\prime}}, \pi\right) .
$$

This is left exact. Let $\lambda_{P^{\prime}}^{+}$be as in Proposition 2.5. By Proposition 2.5, we have $\mathcal{H}_{P^{\prime}}=\mathcal{H}_{P^{\prime}}^{+}\left(T_{\lambda_{P^{\prime}}^{+}}^{P^{\prime}}\right)^{-1}$. Hence $\varphi \mapsto\left(\varphi\left(\left(T_{\lambda_{P^{\prime}}^{+}}^{P^{\prime}}\right)^{-n}\right)\right)$ gives an isomorphism

$$
R_{P}(\pi) \simeq\left\{\left(x_{n}\right)_{n \in \mathbb{Z}_{\geq 1}} \mid x_{n} \in \pi, x_{n+1} T_{\lambda_{P^{\prime}}^{+}}=x_{n}\right\} .
$$

Let $P_{1}$ be a parabolic subgroup containing $P$. Then the left adjoint functor (resp. the right adjoint functor) of $I_{P}^{P_{1}}$ is denoted by $L_{P}^{P_{1}}$ (resp. $R_{P}^{P_{1}}$ ).

5.2. Parabolic inductions and adjoint functors. In this subsection, we prove the following proposition. The condition on $\sigma$ is found in the study in $\mathrm{AHVa}$.

Proposition 5.1. Let $P, Q$ be parabolic subgroups and $\sigma$ an $\mathcal{H}_{Q}$-module. Assume that $\bigcap_{n \in \mathbb{Z}_{\geq 0}} p^{n} \sigma=0$. Then we have $R_{P} \circ I_{Q}(\sigma) \simeq I_{P \cap Q}^{P} \circ R_{P \cap Q}^{Q}(\sigma)$. 
Before proving the proposition, we reformulate the proposition in terms of

$$
\begin{aligned}
\widetilde{I}_{Q}(\sigma) & =\sigma \otimes_{\left(\mathcal{H}_{Q}^{+}, j_{Q}^{+}\right)} \mathcal{H}, \\
\widetilde{R}_{P}(\pi) & =\operatorname{Hom}_{\left(\mathcal{H}_{P}^{+}, j_{P}^{+}\right)}\left(\mathcal{H}_{P}, \pi\right) .
\end{aligned}
$$

By Proposition 2.21, we have

$$
I_{Q}(\sigma)=\widetilde{I}_{Q^{\prime}}\left(n_{w_{G} w_{Q}} \sigma\right), \quad R_{P}(\pi)=n_{w_{G} w_{P}}^{-1} \widetilde{R}_{P^{\prime}}(\pi)
$$

where $P^{\prime}=n_{w_{G} w_{P}} P^{\mathrm{op}} n_{w_{G} w_{P}}^{-1}$ and $Q^{\prime}=n_{w_{G} w_{Q}} Q^{\mathrm{op}} n_{w_{G} w_{Q}}^{-1}$.

Lemma 5.2. Let $P, Q$ be a parabolic subgroup and $\sigma$ an $\mathcal{H}_{Q}$-module. Assume that $\bigcap_{n \in \mathbb{Z}_{\geq 0}} p^{n} \sigma=0$. Then we have $\widetilde{R}_{P} \circ \widetilde{I}_{Q}(\sigma) \simeq \widetilde{I}_{P \cap Q}^{P} \circ \widetilde{R}_{P \cap Q}^{Q}(\sigma)$.

We prove that Lemma 5.2 implies Proposition [5.1. We have

$$
R_{P} \circ I_{Q}=n_{w_{G} w_{P}}^{-1} \widetilde{R}_{P^{\prime}} \circ \widetilde{I}_{Q^{\prime}} n_{w_{G} w_{Q}} .
$$

By Lemma 5.2, we have $\widetilde{R}_{P^{\prime}} \circ \widetilde{I}_{Q^{\prime}}=\widetilde{I}_{P^{\prime} \cap Q^{\prime}}^{P^{\prime}} \circ \widetilde{R}_{P^{\prime} \cap Q^{\prime}}^{Q^{\prime}}$. Let $P_{1}$ be a parabolic subgroup such that $\Delta_{P_{1}}=w_{P}\left(-\Delta_{P \cap Q}\right)=w_{P} w_{P \cap Q}\left(\Delta_{P \cap Q}\right)$. Then we have $w_{G} w_{P}\left(\Delta_{P_{1}}\right)=w_{G}\left(-\Delta_{P \cap Q}\right)=w_{G}\left(-\Delta_{P}\right) \cap w_{G}\left(-\Delta_{Q}\right)=\Delta_{P^{\prime}} \cap$ $\Delta_{Q^{\prime}}=\Delta_{P^{\prime} \cap Q^{\prime}}$. The adjoint action of $n_{w_{G} w_{P}}$ induces an isomorphism $\mathcal{H}_{P} \simeq \mathcal{H}_{P^{\prime}}$ and it induces an isomorphism $\mathcal{H}_{P_{1}}^{P+}$ to $\mathcal{H}_{P^{\prime} \cap Q^{\prime}}^{P^{\prime}+}$. Hence we get $n_{w_{G} w_{P}}^{-1} \widetilde{R}_{P^{\prime} \cap Q^{\prime}}^{P^{\prime}}=\widetilde{R}_{P_{1}}^{P} n_{w_{G} w_{P}}^{-1}$. Similarly we have $\widetilde{I}_{P^{\prime} \cap Q^{\prime}}^{Q^{\prime}} n_{w_{G} w_{Q}}=n_{w_{G} w_{Q}} \widetilde{I}_{P_{2}}^{Q}$ where $P_{2}$ is a parabolic subgroup corresponding to $w_{Q}\left(-\Delta_{P \cap Q}\right)$. Therefore we have

$$
R_{P} \circ I_{Q}=\widetilde{I}_{P_{1}}^{P} n_{w_{G} w_{P}}^{-1} n_{w_{G} w_{Q}} \widetilde{R}_{P_{2}}^{Q} .
$$

Since we have $n_{w_{G} w_{P}} n_{w_{P} w_{P \cap Q}}=n_{w_{G} w_{P \cap Q}}=n_{w_{G} w_{Q}} n_{w_{Q} w_{P \cap Q}}$, we have $n_{w_{G} w_{P}}^{-1} n_{w_{G} w_{Q}}=n_{w_{P} w_{P \cap Q}} n_{w_{Q} w_{P \cap Q}}^{-1}$. Since $w_{P} w_{P \cap Q}\left(\Delta_{P \cap Q}\right)=\Delta_{P_{1}}$, we have $\widetilde{I}_{P_{1}}^{P} n_{w_{P} w_{P \cap Q}}=I_{P \cap Q}^{P}$. Similarly we have $n_{w_{Q} q_{P \cap Q}}^{-1} \widetilde{R}_{P_{2}}^{Q}=R_{P \cap Q}^{Q}$. Hence we get Proposition 5.1.

In the rest of this section, we prove Lemma [5.2. Recall a filtration introduced in subsection 3.1. Let $A \subset{ }^{Q} W_{0}$ be a closed subset and fix a maximal element $w \in A$. Set $A^{\prime}=A \backslash\{w\}$. Then the quotient

$$
\left(\sum_{v \in A} \sigma \otimes T_{n_{v}}^{*}\right) /\left(\sum_{v \in A^{\prime}} \sigma \otimes T_{n_{v}}^{*}\right)
$$

is isomorphic to $\sigma$ as vector spaces and the action of $E_{O_{-}}(\lambda)$ is given by $\widetilde{q}\left(Q, n_{w} \cdot \lambda\right) \sigma\left(E_{o_{-},}^{Q}\left(n_{w} \cdot \lambda\right)\right)$ by Lemma 3.5 where $\widetilde{q}(Q, \mu)=q\left(Q^{\prime}, n_{w_{G} w_{Q}} \cdot \mu\right)$. By Remark [3.7, $\widetilde{q}(Q, \mu) \neq 1$ if and only if $\mu$ is $Q$-positive.

Lemma 5.3. Let $\lambda_{P}^{+}$be as in Proposition [2.5. Then $n_{w} \cdot \lambda_{P}^{+}$is $Q$-positive if and only if $w \in{ }^{Q} W_{0} \cap W_{0, P}={ }^{P \cap Q} W_{0, P}$.

Proof. The element $n_{w} \cdot \lambda_{P}^{+}$is $Q$-positive if and only if $\left\langle\alpha, w \nu\left(\lambda_{P}^{+}\right)\right\rangle \leq 0$ for any $\alpha \in \Sigma^{+} \backslash \Sigma_{Q}^{+}$. Since $\left\langle\beta, \nu\left(\lambda_{P}^{+}\right)\right\rangle \leq 0$ if and only if $\beta \in \Sigma^{+} \cup \Sigma_{P}$, the element $n_{w} \cdot \lambda_{P}^{+}$is $Q$-positive if and only if $w^{-1}\left(\Sigma^{+} \backslash \Sigma_{Q}^{+}\right) \subset \Sigma^{+} \cup \Sigma_{P}$. Since $w \in{ }^{Q} W_{0}$, we have $w^{-1}\left(\Sigma_{Q}^{+}\right) \subset \Sigma^{+}$. Hence this is equivalent to $w^{-1}\left(\Sigma^{+}\right) \subset \Sigma^{+} \cup \Sigma_{P}$. 
Therefore $w^{-1}\left(\Sigma^{-}\right) \supset \Sigma^{-} \backslash \Sigma_{P}^{-}$by taking the complement of the both sides. Hence $w\left(\Sigma^{-} \backslash \Sigma_{P}^{-}\right) \subset \Sigma^{-}$. Therefore we have $w \in W_{0, P}$.

For the last part, ${ }^{Q} W_{0} \cap W_{0, P} \subset{ }^{P \cap Q} W_{0, P}$ is obvious. If $w \in W_{0, P}$, then $w^{-1}\left(\Delta \backslash \Delta_{P}\right) \subset \Sigma^{+}$. Hence if $w \in{ }^{P \cap Q} W_{0, P}$, we have $w^{-1}\left(\left(\Delta \backslash \Delta_{P}\right) \cup\right.$ $\left.\Delta_{P \cap Q}\right) \subset \Sigma^{+}$. We have $\Delta_{Q} \subset\left(\Delta \backslash \Delta_{P}\right) \cup \Delta_{P \cap Q}$. Hence $w \in{ }^{Q} W_{0}$.

Put $I=\sum_{v \in P \cap Q} W_{0, P} \sigma \otimes T_{n_{v}}^{*} \subset \widetilde{I}_{Q}(\sigma)$.

Lemma 5.4. $I=\sum_{v \in W_{0, P}} \sigma \otimes T_{n_{v}}^{*}=\sum_{v \in W_{0, P}} \sigma \otimes T_{n_{v}}$.

Proof. For $v \in W_{0, P}$, Take $v_{1} \in W_{0, P \cap Q}, v_{2} \in{ }^{P \cap Q} W_{0, P}$ such that $v=v_{1} v_{2}$. Then for any $x \in \sigma$ we have $x \otimes T_{n_{v}}^{*}=x T_{n_{v_{1}}}^{Q *} \otimes T_{n_{v_{2}}}^{*}$ since $j_{Q}^{+}\left(T_{n_{v_{2}}}^{Q *}\right)=T_{n_{v_{2}}}^{*}$ by Corollary 2.7. This gives the first equality. The second equality follows from a usual triangular argument.

Lemma 5.5. The subspace $I$ is stable under the action of $E_{o_{-}}(w)$ where $w \in W_{P}(1)$. In particular, I is stable under the action of $j_{P}^{+}\left(\mathcal{H}_{P}^{+}\right)$.

Proof. We prove $\left(\sigma \otimes T_{n_{v}}\right) E_{o_{-}}(w) \subset I$. Take $a \in W_{0, P}$ and $\lambda \in \Lambda(1)$ such that $w=\lambda n_{a}$. Then by the Bernstein relations [Vig16, Corollary 5.43], in $\mathcal{H}\left[q_{s}^{ \pm 1}\right]$, we have

$$
\begin{aligned}
T_{n_{v}} E_{o_{-}}(w) & \in\left(C\left[q_{s}^{ \pm 1}\right] T_{n_{v}} E_{o_{-}}(\lambda) T_{n_{a}}\right) \cap \mathcal{H} \\
& \subset\left(\sum_{b \leq v, \mu \in \Lambda(1)} C\left[q_{s}^{ \pm 1}\right] E_{o_{-}}(\mu) T_{n_{b}} T_{n_{a}}\right) \cap \mathcal{H} .
\end{aligned}
$$

Since $v \in W_{0, P}, b \leq v$ implies $b \in W_{0, P}$. We have $a \in W_{0, P}$. Hence $T_{n_{b}} T_{n_{a}} \in \sum_{c \in W_{0, P}} C\left[Z_{\kappa}\right] T_{n_{c}}$. Therefore

$$
\begin{aligned}
T_{n_{v}} E_{o_{-}}(w) & \in\left(\sum_{c \in W_{0, P}, \mu \in \Lambda(1)} C\left[q_{s}^{ \pm 1}\right] E_{o_{-}}(\mu) T_{n_{c}}\right) \cap \mathcal{H} \\
& =\left(\sum_{c \in W_{0, P}, \mu \in \Lambda(1)} C\left[q_{s}^{ \pm 1}\right] E_{o_{-}}\left(\mu n_{c}\right)\right) \cap \mathcal{H} \\
& =\sum_{c \in W_{0, P}, \mu \in \Lambda(1)} C\left[q_{s}\right] E_{o_{-}}\left(\mu n_{c}\right) .
\end{aligned}
$$

Hence it is sufficient to prove that $\sigma \otimes E_{o_{-}}\left(\mu n_{c}\right) \subset I$, namely we may assume $v=1$. Take $\lambda_{Q}^{+}$as in Proposition 2.5 such that $\lambda_{Q}^{+} \lambda$ is $Q$-positive. Then for $x \in \sigma$, we have

$$
\begin{aligned}
x \otimes E_{o_{-}}\left(\lambda n_{a}\right) & =x \sigma\left(E_{o_{-}, Q}^{Q}\left(\lambda_{Q}^{+}\right)\right)^{-1} \otimes E_{o_{-}}\left(\lambda_{Q}^{+}\right) E_{o_{-}}\left(\lambda n_{a}\right) \\
& \in C x \sigma\left(E_{o_{-, Q}}^{Q}\left(\lambda_{Q}^{+}\right)\right)^{-1} \otimes E_{o_{-}}\left(\lambda_{Q}^{+} \lambda n_{a}\right) .
\end{aligned}
$$

Here we use the product formula (2.1). Therefore we may assume that $\lambda$ is $Q$-positive. Take $a_{1} \in W_{0, P \cap Q}$ and $a_{2} \in{ }^{P \cap Q} W_{0, P}$ such that $a=a_{1} a_{2}$. Then $\lambda n_{a_{1}}$ is $Q$-positive. By Lemma 2.20, we have $\ell\left(\lambda n_{a}\right)=\ell\left(\lambda n_{a_{1}}\right)+$ $\ell\left(n_{a_{2}}\right)$. Hence by (2.1), we have $E_{o_{-}}\left(\lambda n_{a}\right)=E_{o_{-}}\left(\lambda n_{a_{1}}\right) E_{o_{-} \cdot a_{1}}\left(n_{a_{2}}\right)$. By 
Lemma 4.15, we have $E_{o_{-} \cdot a_{1}}\left(n_{a_{2}}\right)=E_{o_{-}}\left(n_{a_{2}}\right)$. Hence

$$
\begin{aligned}
x \otimes E_{o_{-}}\left(\lambda n_{a}\right) & =x \otimes E_{o_{-}}\left(\lambda n_{a_{1}}\right) E_{o_{-}}\left(n_{a_{2}}\right) \\
& =x \sigma\left(E_{o_{-}, Q}^{Q}\left(\lambda n_{a_{1}}\right)\right) \otimes T_{n_{a_{2}}} .
\end{aligned}
$$

We get the lemma.

Consider the exact sequence

$$
0 \rightarrow I \rightarrow I_{Q}(\sigma) \rightarrow I_{Q}(\sigma) / I \rightarrow 0
$$

as $\left(\mathcal{H}_{P}^{+}, j_{P}^{+}\right)$-modules. We have the exact sequence

$$
\begin{aligned}
0 \rightarrow \operatorname{Hom}_{\left(\mathcal{H}_{P}^{+}, j_{P}^{+}\right)}\left(\mathcal{H}_{P}, I\right) \rightarrow \operatorname{Hom}_{\left(\mathcal{H}_{P}^{+}, j_{P}^{+}\right)}\left(\mathcal{H}_{P}, I_{Q}(\sigma)\right) \\
\\
\rightarrow \operatorname{Hom}_{\left(\mathcal{H}_{P}^{+}, j_{P}^{+}\right)}\left(\mathcal{H}_{P}, I_{Q}(\sigma) / I\right) .
\end{aligned}
$$

Lemma 5.6. Assume that $\bigcap_{n} p^{n} \sigma=0$. Then $\operatorname{Hom}_{\left(\mathcal{H}_{P}^{+}, j_{P}^{+}\right)}\left(\mathcal{H}_{P}, I_{Q}(\sigma) / I\right)=$ 0 .

Proof. Let $\lambda_{P}^{+}$as in Proposition 2.5. By Lemma 2.6, $j_{P}^{+}\left(E_{o_{-}, P}^{P}\left(\lambda_{P}^{+}\right)\right)=$ $E_{o_{-}}\left(\lambda_{P}^{+}\right)$. Hence by Proposition 2.5, we have

$$
\operatorname{Hom}_{\left(\mathcal{H}_{P}^{+}, j_{P}^{+}\right)}\left(\mathcal{H}_{P}, I_{Q}(\sigma) / I\right)=\operatorname{Hom}_{C\left[E_{o_{-}}\left(\lambda_{P}^{+}\right)\right]}\left(C\left[E_{o_{-}}\left(\lambda_{P}^{+}\right)^{ \pm 1}\right], I_{Q}(\sigma) / I\right) .
$$

Define a $C\left[E_{o_{-}}\left(\lambda_{P}^{+}\right)\right]$-module $\sigma_{w}$ by

$$
\sigma_{w}\left(E_{o_{-}}\left(\lambda_{P}^{+}\right)\right)=\widetilde{q}\left(P, n_{w} \cdot \lambda\right) e_{Q}(\sigma)\left(E_{o_{-}, Q}^{Q}\left(n_{w} \cdot \lambda_{P}^{+}\right)\right)
$$

on the same space as $\sigma$. Then $I_{Q}(\sigma) / I$ has a $C\left[E_{o_{-}}\left(\lambda_{P}^{+}\right)\right]$-stable filtration whose subquotient is given by $\sigma_{w}$ where $w \in{ }^{Q} W_{0} \backslash P \cap Q{ } W_{0, P}$. By Lemma 5.3, $\widetilde{q}\left(P, n_{w} \cdot \lambda\right) \neq 1$. Hence it is a positive power of $p$. Therefore the image of $\psi \in \operatorname{Hom}_{C\left[E_{o_{-}}\left(\lambda_{P}^{+}\right)\right]}\left(C\left[E_{o_{-}}\left(\lambda_{P}^{+}\right)^{ \pm 1}\right], \sigma_{w}\right)$ is contained in $\bigcap_{n \geq 0} p^{n} \sigma_{w}$. This is zero by the assumption.

Hence to prove Lemma 5.2 , it is sufficient to prove

$$
\operatorname{Hom}_{\left(\mathcal{H}_{P}^{+}, j_{P}^{+}\right)}\left(\mathcal{H}_{P}, I\right) \simeq \operatorname{Hom}_{\left(\mathcal{H}_{P \cap Q}^{Q+}, j_{P \cap Q}^{Q+}\right)}\left(\mathcal{H}_{P \cap Q}, \sigma\right) \otimes_{\left(\mathcal{H}_{P \cap Q}^{P+}, j_{P \cap Q}^{P+}\right)} \mathcal{H}_{P}
$$

To construct a homomorphism from the right hand side of (5.1) to the left hand side, it is sufficient to construct an $\left(\mathcal{H}_{P \cap Q}^{P+}, j_{P \cap Q}^{P+}\right)$-homomorphism $\operatorname{Hom}_{\left(\mathcal{H}_{P \cap Q}^{Q+}, j_{P \cap Q}^{Q+}\right)}\left(\mathcal{H}_{P \cap Q}, \sigma\right) \rightarrow \operatorname{Hom}_{\left(\mathcal{H}_{P}^{+}, j_{P}^{+}\right)}\left(\mathcal{H}_{P}, I\right)$. Let $\lambda_{P}^{+}$as in Proposition 2.5. By Lemma 3.16, we have

$$
\left.\operatorname{Hom}_{\left(\mathcal{H}_{P}^{+}, j_{P}^{+}\right)}\left(\mathcal{H}_{P}, I\right)\right|_{\left(\mathcal{H}_{P \cap Q}^{P+}, j_{P \cap Q}^{P+}\right)} \simeq \operatorname{Hom}_{\left(\mathcal{H}_{P \cap Q}^{+}, j_{P \cap Q}^{+}\right)}\left(\mathcal{H}_{P \cap Q}^{P+}, I\right) .
$$

(Both sides are isomorphic to $\operatorname{Hom}_{C\left[E_{o_{-}}\left(\lambda_{P}^{+}\right)\right]}\left(C\left[E_{o_{-}}\left(\lambda_{P}^{+}\right)^{ \pm 1}\right], I\right)$.) Now the restriction to from $\mathcal{H}_{P \cap Q}$ to $\mathcal{H}_{P \cap Q}^{P+}$ gives a map $\operatorname{Hom}_{\left(\mathcal{H}_{P \cap Q}^{Q+}, j_{P \cap Q}^{Q+}\right)}\left(\mathcal{H}_{P \cap Q}, \sigma\right) \rightarrow$ $\operatorname{Hom}_{\left(\mathcal{H}_{P \cap Q}^{+}, j_{P \cap Q}^{Q+}\right)}\left(\mathcal{H}_{P \cap Q}^{P+}, \sigma\right)$. Combining the inclusion $\sigma=\sigma \otimes 1 \hookrightarrow I$ which is $\left(\mathcal{H}_{Q}^{+}, j_{Q}^{+}\right)$-equivariant, we get

$$
\begin{aligned}
\operatorname{Hom}_{\left(\mathcal{H}_{P \cap Q}^{Q+}, j_{P \cap Q}^{Q+}\right)}\left(\mathcal{H}_{P \cap Q}, \sigma\right) \rightarrow \operatorname{Hom}_{\left(\mathcal{H}_{P \cap Q}^{+}, j_{P \cap Q}^{+}\right)}\left(\mathcal{H}_{P \cap Q}^{P+}, I\right) \\
\\
\simeq \operatorname{Hom}_{\left(\mathcal{H}_{P}^{+}, j_{P}^{+}\right)}\left(\mathcal{H}_{P}, I\right)
\end{aligned}
$$


and it gives a homomorphism between (5.1).

We prove that this gives an isomorphism. We have the decomposition

$$
I=\bigoplus_{v \in P \cap Q_{W_{0, P}}} \sigma \otimes T_{n_{v}}
$$

We have $\left\langle\alpha, \nu\left(\lambda_{P}^{+}\right)\right\rangle=0$ for any $\alpha \in \Delta_{P}$. Hence the Bernstein relations Vig16, Corollary 5.43] tells that $T_{n_{v}} E_{o_{-}}\left(\lambda_{P}^{+}\right)=E_{o_{-}}\left(n_{v} \cdot \lambda_{P}^{+}\right) T_{n_{v}}=$ $E_{o_{-}}\left(\lambda_{P}^{+}\right) T_{n_{v}}$ for any $v \in W_{0, P}$. (For the last part, recall that $\lambda_{P}^{+}$is in the center of $W_{P}(1)$.) Therefore each summand $\sigma \otimes T_{n_{v}}$ is stable under the action of $E_{o_{-}}\left(\lambda_{P}^{+}\right)$and we have

$$
\operatorname{Hom}_{\left(\mathcal{H}_{P}^{+}, j_{P}^{+}\right)}\left(\mathcal{H}_{P}, I\right) \simeq \bigoplus_{v \in P \cap Q} W_{0, P} \operatorname{Hom}_{C\left[E_{o_{-}}\left(\lambda_{P}^{+}\right)\right]}\left(C\left[E_{o_{-}}\left(\lambda_{P}^{+}\right)^{ \pm 1}\right], \sigma \otimes T_{n_{v}}\right) .
$$

For $x \in \sigma$, from the above calculation we have $x \otimes T_{n_{v}} E_{o_{-}}\left(\lambda_{P}^{+}\right)=x \otimes$ $E_{o_{-}}\left(\lambda_{P}^{+}\right) T_{n_{v}}=x E_{o_{-}, Q}^{Q}\left(\lambda_{P}^{+}\right) \otimes T_{n_{v}}$. Hence each summand is isomorphic to $\operatorname{Hom}_{C\left[E_{o_{-}, Q}^{Q}\left(\lambda_{P}^{+}\right)\right]}\left(C\left[E_{o_{-, Q}}^{Q}\left(\lambda_{P}^{+}\right)^{ \pm 1}\right], \sigma\right)$. Therefore we have

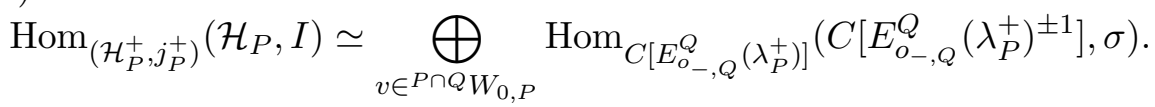

On the other hand, the right hand side of (5.1) is

$$
\bigoplus_{v \in P \cap Q W_{0, P \cap Q}} \operatorname{Hom}_{\left(\mathcal{H}_{P \cap Q}^{Q+}, j_{P \cap Q}^{Q+}\right)}\left(\mathcal{H}_{P \cap Q}, \sigma\right) \otimes T_{n_{v}} .
$$

Lemma 5.7. The element $\lambda_{P}^{+}$satisfies the condition of $\lambda_{P \cap Q}^{Q+}$ in Proposition 2.5.

Proof. The element $\lambda_{P}^{+} \in Z\left(W_{P}(1)\right)$ satisfies $\left\langle\alpha, \nu\left(\lambda_{P}^{+}\right)\right\rangle<0$ for any $\alpha \in \Sigma^{+} \backslash$ $\Sigma_{P}^{+}$. Hence $\left\langle\alpha, \nu\left(\lambda_{P}^{+}\right)\right\rangle<0$ for any $\alpha \in \Sigma_{Q}^{+} \backslash \Sigma_{P \cap Q}^{+}$and $\lambda_{P}^{+} \in Z\left(W_{P \cap Q}(1)\right)$.

Hence we have $\mathcal{H}_{P \cap Q}=\mathcal{H}_{P \cap Q}^{Q+} E_{o_{-}, P \cap Q}^{P \cap Q}\left(\lambda_{P}^{+}\right)^{-1}$. Note that, by Proposition 2.6, we have $j_{P \cap Q}^{Q+}\left(E_{o_{-}, P \cap Q}^{P \cap Q}\left(\lambda_{P}^{+}\right)\right)=E_{o_{-}, Q}^{Q}\left(\lambda_{P}^{+}\right)$. Therefore (5.3) is equal to

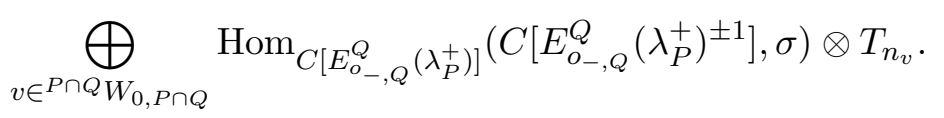

This is isomorphic to (5.2). This ends the proof of Lemma 5.2,

Corollary 5.8. Assume that $p$ is nilpotent in $C$. Then we have $L_{P} \circ I_{Q} \simeq$ $I_{P}^{P \cap Q} \circ L_{P \cap Q}^{P}$.

Proof. If $p$ is nilpotent, then any module satisfies $\bigcap_{n} p^{n} \sigma=0$. Hence we have $R_{P} \circ I_{Q} \simeq I_{P \cap Q}^{P} \circ R_{P \cap Q}^{P}$. Taking the left adjoint functors of the both sides, we get the corollary.

5.3. $L_{P}$ and Steinberg modules. In this section, we fix a parabolic subgroup $P \subset G$ such that $\Delta_{P}$ and $\Delta \backslash \Delta_{P}$ are orthogonal to each other. Let $\sigma$ be an $\mathcal{H}_{P}$-module which has the extension $e(\sigma)$ to $\mathcal{H}$. Then we have an $\mathcal{H}$-module $\operatorname{St}_{Q}(\sigma)$ for $Q \supset P$. Let $R$ be another parabolic subgroup.

Lemma 5.9. We have $L_{R}\left(e_{G}(\sigma)\right) \simeq e_{R}\left(L_{P \cap R}^{P}(\sigma)\right)$. 
Proof. Let $\lambda_{R}^{-}$as in Proposition 2.5. Then by Lemma [5.7, this satisfies the condition of $\lambda_{R \cap P}^{P-}$. Hence we have $L_{P \cap R}^{P}(\sigma)=\sigma E_{o_{-, P}}^{P}\left(\lambda_{R}^{-}\right)^{-1}$. By the definition, $\lambda_{R}^{-}$is dominant, hence in particular, it is $P$-negative. Hence $e_{G}(\sigma)\left(E_{o_{-}}\left(\lambda_{R}^{-}\right)\right)=e_{G}(\sigma)\left(j_{P}^{-*}\left(E_{o_{-}, P}^{P}\left(\lambda_{R}^{-}\right)\right)\right)=\sigma\left(E_{o_{-}, P}^{P}\left(\lambda_{R}^{-}\right)\right)$by Lemma 2.6. Therefore, as vector spaces, we have

$$
L_{R}\left(e_{G}(\sigma)\right)=e_{G}(\sigma) E_{o_{-}}\left(\lambda_{R}^{-}\right)^{-1} \simeq \sigma E_{o_{-, P}}^{P}\left(\lambda_{R}^{-}\right)^{-1}=L_{P \cap R}^{P}(\sigma) .
$$

Namely, the linear map defined by $e_{G}(\sigma) \otimes_{\left(\mathcal{H}_{R}^{-}, j_{R}^{-*}\right)} \mathcal{H}_{R} \ni x \otimes E_{o_{-, R}}^{R}\left(\lambda_{R}^{-}\right)^{-n} \mapsto$ $x \otimes E_{o_{-}, P \cap R}^{P \cap R}\left(\lambda_{R}^{-}\right)^{-n} \in \sigma \otimes_{\left(\mathcal{H}_{P \cap R}^{P-}, j_{P \cap R}^{P-*}\right)} \mathcal{H}_{P \cap R}$ is an isomorphism.

We prove that this map is $\left(\mathcal{H}_{P \cap R}^{R-}, j_{P \cap R}^{R-*}\right)$-equivariant. Let $w \in W_{P \cap R}^{R}(1)$ and take $k \in \mathbb{Z}_{\geq 0}$ such that $w\left(\lambda_{R}^{-}\right)^{k}$ is $R$-negative. Since the length of $\lambda_{R}^{-}$ as an element of $W_{R}(1)$ is zero, we have $T_{w}^{R *} E_{o_{-, R}}^{R}\left(\lambda_{R}^{-}\right)^{k}=T_{w}^{R *} T_{\left(\lambda_{R}^{-}\right)^{k}}^{R *}=$ $T_{w\left(\lambda_{R}^{-}\right)^{k}}^{R *}$. We also have that $E_{o_{-, R}}^{R}\left(\lambda_{R}^{-}\right)$is in the center of $\mathcal{H}_{R}^{-}$. Replacing $R$ with $P \cap R$, we also have $T_{w}^{(P \cap R) *} E_{o_{-}, P \cap R}^{P \cap R}\left(\lambda_{R}^{-}\right)^{k}=T_{w\left(\lambda_{R}^{-}\right)^{k}}^{(P \cap R) *}$. Hence for $x \in \sigma$, we have

$$
\begin{aligned}
& e_{G}(\sigma) \otimes_{\left(\mathcal{H}_{R}^{-}, j_{R}^{-*}\right)} \mathcal{H}_{R} \ni x \otimes E_{o_{-, R}}^{R}\left(\lambda_{R}^{-}\right)^{-n} T_{w}^{R *} \\
& =x \otimes T_{w}^{R *} E_{o_{-, R}}^{R}\left(\lambda_{R}^{-}\right)^{-n} \\
& =x \otimes T_{w\left(\lambda_{R}^{-}\right)^{k}}^{R *} E_{o_{-, R}}^{R}\left(\lambda_{R}^{-}\right)^{-(n+k)} \\
& =x e_{G}(\sigma)\left(T_{w\left(\lambda_{R}^{-}\right)^{k}}^{*}\right) \otimes E_{o_{-, R}}^{R}\left(\lambda_{R}^{-}\right)^{-(n+k)} \\
& =x \sigma\left(T_{w\left(\lambda_{R}^{-}\right)^{k}}^{P *}\right) \otimes E_{o_{-, R}}^{R}\left(\lambda_{R}^{-}\right)^{-(n+k)} \\
& \mapsto x \sigma\left(T_{w\left(\lambda_{R}^{-}\right)^{k}}^{P *}\right) \otimes E_{o_{-}, P \cap R}^{P \cap R}\left(\lambda_{R}^{-}\right)^{-(n+k)} \\
& =x \otimes T_{w\left(\lambda_{R}^{-}\right)^{k}}^{(P \cap R) *} E_{o_{-, P \cap R}}^{P \cap R}\left(\lambda_{R}^{-}\right)^{-(n+k)} \\
& =x \otimes E_{o_{-, P \cap R}}^{P \cap R}\left(\lambda_{R}^{-}\right)^{-n} T_{w}^{(P \cap R) *} \\
& \in \sigma \otimes_{\left(\mathcal{H}_{P \cap R}^{P-}, j_{P \cap R}^{P-*}\right)} \mathcal{H}_{P \cap R}
\end{aligned}
$$

Therefore the above homomorphism is $\left(\mathcal{H}_{P \cap R}^{R-}, j_{P \cap R}^{R-*}\right)$-equivariant.

Let $P_{2}$ be a parabolic subgroup corresponding to $\Delta \backslash \Delta_{P}$. Fix $w \in$ $W_{P_{2} \cap R \text {,aff }}(1)$ and take $v \in W_{0}$ and $\lambda \in \Lambda(1)$ such that $w=n_{v} \lambda$. Then we have $\nu(\lambda) \in \mathbb{R}\left(\Delta_{P_{2}}^{\vee} \cap \Delta_{R}^{\vee}\right)$. In particular, $\langle\alpha, \nu(\lambda)\rangle=0$ for any $\alpha \in \Delta_{P}$. Take a dominant $\mu \in Z\left(W_{R}(1)\right) \cap W_{P_{2} \text {,aff }}(1)$ such that $\langle\alpha, \nu(\mu)\rangle=0$ for any $\alpha \in \Delta_{R} \cup \Delta_{P}$ and $\langle\alpha, \nu(\mu)\rangle$ is sufficiently large for $\alpha \in \Delta \backslash\left(\Delta_{R} \cup \Delta_{P}\right)=$ $\Delta_{P_{2}} \backslash \Delta_{R}$. Then $\langle\alpha, \nu(\lambda \mu)\rangle=0$ for any $\alpha \in \Delta_{P}$. For $\alpha \in \Sigma_{P_{2}}^{+} \backslash \Sigma_{R}^{+}$, we can take $\mu$ such that $\langle\alpha, \nu(\lambda \mu)\rangle \geq 0$. For such $\mu$, we have $\langle\alpha, \nu(\lambda \mu)\rangle \geq 0$ for any $\alpha \in \Sigma_{P}^{+} \cup\left(\Sigma_{P_{2}}^{+} \backslash \Sigma_{R}^{+}\right)=\Sigma^{+} \backslash \Sigma_{R}^{+}$. Namely we can take $\mu$ such that $\lambda \mu$ is $R$-negative. Since $w \mu$ is $R$-negative, for $x \in \sigma$, we have

$x \otimes E_{o_{-, R}}^{R}\left(\lambda_{R}^{-}\right)^{-n} T_{w \mu}^{R *}=x e_{G}(\sigma)\left(T_{w \mu}^{*}\right) \otimes E_{o_{-, R}}^{R}\left(\lambda_{R}^{-}\right)^{-n} \in e_{G}(\sigma) \otimes_{\left(\mathcal{H}_{R}^{-}, j_{R}^{-*}\right)} \mathcal{H}_{R}$.

Since $w \mu \in W_{P_{2} \text {,aff }}(1), e_{G}(\sigma)\left(T_{w \mu}^{*}\right)=1$. Hence $L_{R}\left(e_{G}(\sigma)\right)\left(T_{w \mu}^{R *}\right)=1$. In particular, by taking $w=1$, we have $L_{R}\left(e_{G}(\sigma)\right)\left(T_{\mu}^{R *}\right)=1$. Since $\mu \in$ 
$Z\left(W_{R}(1)\right)$, we have $T_{w \mu}^{R *}=T_{w}^{R *} T_{\mu}^{R *}$. Therefore we get $L_{R}\left(e_{G}(\sigma)\right)\left(T_{w}^{R *}\right)=1$. By the characterization of $e_{R}\left(L_{P \cap R}^{P}(\sigma)\right)$, we get the lemma.

Proposition 5.10. Assume that $p$ is nilpotent in $C$. We have

$$
L_{R}\left(\operatorname{St}_{Q}(\sigma)\right)= \begin{cases}\operatorname{St}_{Q \cap R}^{R}\left(L_{P \cap R}^{P}(\sigma)\right) & \left(\Delta_{Q} \cup \Delta_{R}=\Delta\right), \\ 0 & (\text { otherwise }) .\end{cases}
$$

Proof. We have an exact sequence

$$
\bigoplus_{Q^{\prime} \supsetneq Q} I_{Q^{\prime}}\left(e_{Q^{\prime}}(\sigma)\right) \rightarrow I_{Q}\left(e_{Q}(\sigma)\right) \rightarrow \operatorname{St}_{Q}(\sigma) \rightarrow 0 .
$$

Since $L_{R}$ is exact, we have

$$
\bigoplus_{Q^{\prime} \ni Q} L_{R}\left(I_{Q^{\prime}}\left(e_{Q^{\prime}}(\sigma)\right)\right) \rightarrow L_{R}\left(I_{Q}\left(e_{Q}(\sigma)\right)\right) \rightarrow L_{R}\left(\operatorname{St}_{Q}(\sigma)\right) \rightarrow 0 .
$$

By Corollary [5.8, we have

$$
\bigoplus_{Q^{\prime} \supsetneq Q} I_{Q^{\prime} \cap R}^{R}\left(L_{Q^{\prime} \cap R}^{Q^{\prime}}\left(e_{Q^{\prime}}(\sigma)\right)\right) \rightarrow I_{Q \cap R}^{R}\left(L_{Q \cap R}^{Q}\left(e_{Q}(\sigma)\right)\right) \rightarrow L_{R}\left(\operatorname{St}_{Q}(\sigma)\right) \rightarrow 0 .
$$

By Lemma 5.9, we have

$$
\bigoplus_{Q^{\prime} \supsetneq Q} I_{Q^{\prime} \cap R}^{R}\left(e_{Q^{\prime} \cap R}\left(L_{P \cap R}^{P}(\sigma)\right)\right) \rightarrow I_{Q \cap R}^{R}\left(e_{Q \cap R}\left(L_{P \cap R}^{P}(\sigma)\right)\right) \rightarrow L_{R}\left(\operatorname{St}_{Q}(\sigma)\right) \rightarrow 0 .
$$

Hence if there exists $Q^{\prime} \supsetneq Q$ such that $Q^{\prime} \cap R=Q \cap R$, then $L_{R}\left(\operatorname{St}_{Q}(\sigma)\right)=0$. Such $Q^{\prime}$ exists if and only if $\Delta_{Q} \cup \Delta_{R} \neq \Delta$. Therefore, if $\Delta_{Q} \cup \Delta_{R} \neq \Delta$, then $L_{R}\left(\operatorname{St}_{Q}(\sigma)\right)=0$. If $\Delta_{Q} \cup \Delta_{R}=\Delta$ then $\left\{Q^{\prime} \cap R \mid Q^{\prime} \supsetneq Q\right\}=\left\{Q^{\prime \prime} \mid\right.$ $\left.R \supset Q^{\prime \prime} \supsetneq Q \cap R\right\}$. Therefore we get $L_{R}\left(\operatorname{St}_{Q}(\sigma)\right) \simeq \operatorname{St}_{Q \cap R}^{R}\left(L_{P \cap R}^{P}(\sigma)\right)$.

5.4. $R_{P}$ and an exactness. In the next subsection, we will prove the following proposition.

Proposition 5.11. Let $P$ be a parabolic subgroup, $\sigma$ an $\mathcal{H}_{P}$-module which has the extension to $\mathcal{H}$ and $Q$ a parabolic subgroup containing $P$. Assume that $\bigcap_{n \in \mathbb{Z}_{\geq 0}} p^{n} \sigma=0$. Then for any parabolic subgroup $R$, we have

$$
R_{R}\left(\operatorname{St}_{Q}(\sigma)\right)= \begin{cases}\operatorname{St}_{Q \cap R}^{R}\left(R_{P \cap R}^{P}(\sigma)\right) & \left(\Delta_{Q}=\Delta_{Q \cap R} \cup \Delta_{P}\right) \\ 0 & (\text { otherwise }) .\end{cases}
$$

The proof of this proposition is similar to that of Proposition 5.10. However, to use that argument, we need the following lemma. Note that this is not obvious since $R_{R}$ is not right exact.

Lemma 5.12. Let $P$ be a parabolic subgroup, $\sigma$ an $\mathcal{H}_{P}$-module which has the extension to $\mathcal{H}$ and $Q$ a parabolic subgroup containing $P$. Then for any parabolic subgroup $R$, the sequence

$$
\bigoplus_{Q_{1} \supsetneq Q} R_{R}\left(I_{Q_{1}}\left(e_{Q_{1}}(\sigma)\right)\right) \rightarrow R_{R}\left(I_{Q}\left(e_{Q}(\sigma)\right)\right) \rightarrow R_{R}\left(\operatorname{St}_{Q}(\sigma)\right) \rightarrow 0
$$

is exact.

We remark that we do not assume that $\bigcap_{n} p^{n} \sigma=0$. The aim of this subsection is to prove this lemma. 
Lemma 5.13. To prove Lemma 5.12, we may assume $\sigma=\mathbf{1}$ and $R$ contains $P$.

Proof. Put $R^{\prime}=n_{w_{G} w_{R}} R^{\mathrm{op}} n_{w_{G} w_{R}}^{-1}$. Let $R_{1}^{\prime}$ (resp. $R_{2}^{\prime}$ ) be a parabolic subgroup corresponding to $\Delta_{R^{\prime}} \cup \Delta_{P}$ (resp. $\Delta_{R^{\prime}} \cup \Delta_{P_{2}}$ ). Let $\lambda_{1}=\lambda_{R_{1}^{\prime}}^{+}$and $\lambda_{2}=\lambda_{R_{2}^{\prime}}^{+}$be as in Proposition 2.5. Moreover we take $\lambda_{1}$ from $W_{P_{2} \text {,aff }}(1)$ and $\lambda_{2}$ from $W_{P, \text { aff }}(1)$. Then $\lambda_{1} \lambda_{2}$ satisfies the condition of $\lambda_{R^{\prime}}^{+}$. We have

$$
R_{R}(\pi)=n_{w_{G} w_{R}}^{-1} \operatorname{Hom}_{\left(\mathcal{H}_{R^{\prime}}^{+}, j_{R^{\prime}}^{+}\right.}\left(\mathcal{H}_{R^{\prime}}, \pi\right) .
$$

Since $\mathcal{H}_{R^{\prime}}=\mathcal{H}_{R^{\prime}}\left(T_{\lambda_{R^{\prime}}^{+}}^{R^{\prime}}\right)^{-1}$, we have

$$
R_{R}(\pi) \simeq \operatorname{Hom}_{C\left[T_{\lambda_{R^{\prime}}^{+}}\right]}\left(C\left[\left(T_{\lambda_{R^{\prime}}^{+}}\right)^{ \pm 1}\right], \pi\right)
$$

as vector spaces. Therefore we have

$$
R_{R}(\pi) \simeq \operatorname{Hom}_{C\left[T_{\lambda_{1}}\right]}\left(C\left[\left(T_{\lambda_{1}}\right)^{ \pm 1}\right], \operatorname{Hom}_{C\left[T_{\lambda_{2}}\right]}\left(C\left[\left(T_{\lambda_{2}}\right)^{ \pm 1}\right], \pi\right)\right) .
$$

Take $\pi=I_{Q}\left(e_{Q}(\sigma)\right)$. Then $I_{Q}\left(e_{Q}(\sigma)\right) \simeq I_{Q}(\mathbf{1}) \otimes e_{G}(\sigma)$ by Lemma 3.17 and $\left(x_{1} \otimes x_{2}\right) T_{\lambda_{1}}=x_{1} T_{\lambda_{1}} \otimes x_{2}$ and $\left(x_{1} \otimes x_{2}\right) T_{\lambda_{2}}=x_{1} \otimes x_{2} T_{\lambda_{2}}$ for $x_{1} \in I_{Q}(\mathbf{1})$ and $x_{2} \in e_{G}(\sigma)$ by Remark 3.14. Hence we get

$$
\begin{aligned}
& R_{R}\left(I_{Q}\left(e_{Q}(\sigma)\right)\right) \\
& \simeq \operatorname{Hom}_{C\left[T_{\lambda_{1}}\right]}\left(C\left[\left(T_{\lambda_{1}}\right)^{ \pm 1}\right], I_{Q}(\mathbf{1})\right) \otimes \operatorname{Hom}_{C\left[T_{\lambda_{2}}\right]}\left(C\left[\left(T_{\lambda_{2}}\right)^{ \pm 1}\right], e_{G}(\sigma)\right) .
\end{aligned}
$$

Since we also have the same formula for $I_{Q_{1}}\left(e_{Q_{1}}(\sigma)\right)$ where $Q_{1} \supsetneq Q$ and $\mathrm{St}_{Q} \sigma$, the sequence in Lemma 5.12 is equal to the sequence

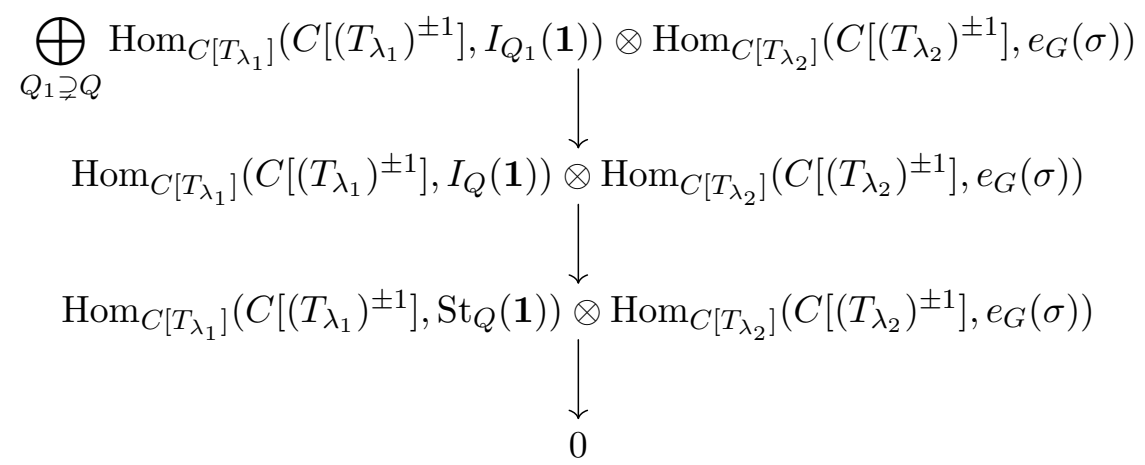

Hence it is sufficient to prove that the exact sequence $\bigoplus_{Q_{1} \supsetneq Q} I_{Q_{1}}(\mathbf{1}) \rightarrow$ $I_{Q}(\mathbf{1}) \rightarrow \operatorname{St}_{Q}(\mathbf{1}) \rightarrow 0$ is still exact after applying $\operatorname{Hom}_{C\left[T_{\lambda_{1}}\right]}\left(C\left[\left(T_{\lambda_{1}}\right)^{ \pm 1}\right], \cdot\right)$. Let $R_{1}$ be a parabolic subgroup corresponding to $\Delta_{R} \cup \Delta_{P}$. Then we have $R_{1}^{\prime}=n_{w_{G} w_{R_{1}}} R_{1}^{\mathrm{op}} n_{w_{G} w_{R_{1}}}^{-1}$ and $\operatorname{Hom}_{C\left[T_{\lambda_{1}}\right]}\left(C\left[\left(T_{\lambda_{1}}\right)^{ \pm 1}\right], \cdot\right) \simeq R_{R_{1}}$ as vector spaces. Hence we may assume $\sigma=\mathbf{1}$ and $R=R_{1}$, namely $R$ contains $P$.

We prove Lemma 5.12 assuming $\sigma=\mathbf{1}$ and $R$ contains $P$. Let $A \subset W_{0}^{Q}$ be an open subset and $w \in A$ a minimal element and $I_{Q}(\mathbf{1})_{A}$ a filtration defined in subsection 3.1. Set $A^{\prime}=A \backslash\{w\}$. As in Remark 3.9, put $I_{Q_{1}, A}=$ 
$I_{Q_{1}}(\mathbf{1}) \cap I_{Q}(\mathbf{1})_{A}$ and let $\operatorname{St}_{Q, A}$ be the image of $I_{Q}(\mathbf{1})_{A}$ in $\operatorname{St}_{Q}(\mathbf{1})$. Then we have a commutative diagram with exact rows and columns (Remark 3.9):

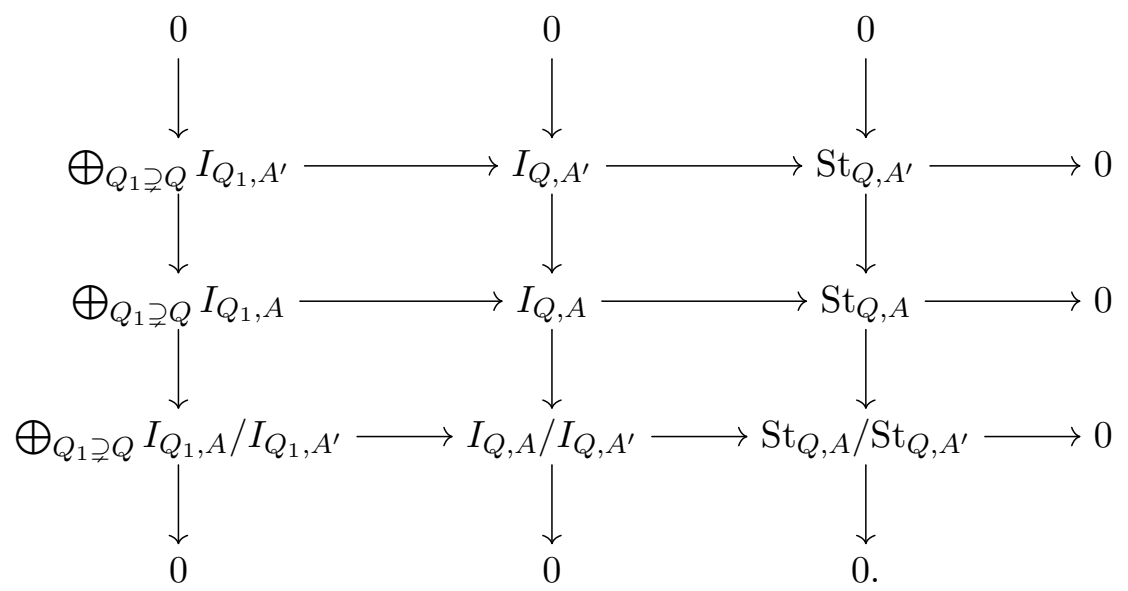

It is sufficient to prove that this diagram remains exact after applying $R_{R}$. By induction on $\# A$, it is sufficient to prove that $R_{R}$ preserves of the exactness of the following three sequences.

$$
\begin{gathered}
\bigoplus_{Q_{1} \supsetneq Q} I_{Q_{1}, A} / I_{Q_{1}, A^{\prime}} \rightarrow I_{Q, A} / I_{Q, A^{\prime}} \rightarrow \mathrm{St}_{Q, A} / \mathrm{St}_{Q, A^{\prime}} \rightarrow 0, \\
0 \rightarrow I_{Q_{1}, A^{\prime}} \rightarrow I_{Q_{1}, A} \rightarrow I_{Q_{1}, A} / I_{Q_{1}, A^{\prime}} \rightarrow 0 \quad\left(Q_{1} \supset Q\right) \\
\quad 0 \rightarrow \mathrm{St}_{Q, A^{\prime}} \rightarrow \mathrm{St}_{Q, A} \rightarrow \mathrm{St}_{Q, A} / \mathrm{St}_{Q, A^{\prime}} \rightarrow 0 .
\end{gathered}
$$

First we prove that $R_{R}$ preserves the exactness of (5.4). Assume that $w \notin$ $W_{0}^{Q_{1}}$ for any $Q_{1} \supsetneq Q$. Then $\bigoplus_{Q_{1} \supsetneq Q} I_{Q_{1}, A} / I_{Q_{1}, A^{\prime}}=0$ by Lemma 3.10, Hence $I_{Q, A} / I_{Q, A^{\prime}} \stackrel{\sim}{\rightarrow} \mathrm{St}_{Q, A} / \mathrm{St}_{Q, A^{\prime}}$. Therefore $R_{R}$ preserves the exactness of (5.4). If $w \in W_{0}^{Q_{1}}$ for some $Q_{1} \supsetneq Q$, then $I_{Q_{1}, A} / I_{Q_{1}, A^{\prime}} \simeq I_{Q, A} / I_{Q, A^{\prime}}$ for such $Q_{1}$ by Lemma 3.10. Hence $\operatorname{St}_{Q, A} / \mathrm{St}_{Q, A^{\prime}}=0$. Moreover, fix $Q_{0} \supsetneq Q$ such that $w \in W_{0}^{Q_{0}}$. Then $I_{Q, A} / I_{Q, A^{\prime}} \simeq I_{Q_{0}, A} / I_{Q_{0}, A^{\prime}} \hookrightarrow \bigoplus_{Q_{1} \supset Q} I_{Q_{1}, A} / I_{Q_{1}, A^{\prime}}$ is a splitting of $\bigoplus_{Q_{1} \supsetneq Q} I_{Q_{1}, A} / I_{Q_{1}, A^{\prime}} \rightarrow I_{Q, A} / I_{Q, A^{\prime}}$. Hence (5.4) is exact after applying $R_{R}$.

Set $R^{\prime}=n_{w_{G} w_{R}} R^{\mathrm{op}} n_{w_{G} w_{R}}^{-1}$ and let $\lambda_{R^{\prime}}^{+}$be as in Proposition 2.5. To prove that $R_{R}$ preserves the exactness of (5.5) and (5.6), we analyze the action of $X=T_{\lambda_{R^{\prime}}^{+}}$on $I_{Q_{1}, A} / I_{Q_{1}, A^{\prime}}$ for $Q_{1} \supset Q$.

Lemma 5.14. The action of $X=T_{\lambda_{R^{\prime}}^{+}}$on $I_{Q_{1}, A} / I_{Q_{1}, A^{\prime}}$ is a power of $p$ and it is 1 if and only if $w_{G}\left(\Sigma_{Q}\right) \subset \Sigma_{R^{\prime}}$ and $w \in W_{0, R^{\prime}} w_{G} w_{Q}$.

Proof. We may assume $Q_{1}=Q$ by Lemma 3.10. Note that since $\lambda_{R^{\prime}}^{+}$is anti-dominant, we have $E_{o_{-}}\left(\lambda_{R^{\prime}}^{+}\right)=T_{\lambda_{R^{\prime}}^{+}}$. Hence by Proposition [3.2, the action of $T_{\lambda_{R^{\prime}}^{+}}=E_{o_{-}}\left(\lambda_{R^{\prime}}^{+}\right)$is given by $q\left(Q, n_{w}^{-1} \cdot \lambda_{R^{\prime}}^{+}\right) \mathbf{1}\left(E_{o_{-}}^{Q}\left(n_{w}^{-1} \cdot \lambda_{R^{\prime}}^{+}\right)\right)$. Since $w \in W_{0}^{Q}, w\left(\Sigma_{Q}^{+}\right) \subset \Sigma^{+}$. Therefore $n_{w}^{-1} \cdot \lambda_{R^{\prime}}^{+}$is anti-dominant with respect to $\Sigma_{Q}^{+}$since $\lambda_{R^{\prime}}^{+}$is anti-dominant with respect to $\Sigma^{+}$. Hence $\mathbf{1}\left(E_{o_{-}}^{Q}\left(n_{w}^{-1} \cdot \lambda_{R^{\prime}}^{+}\right)\right)=$ $\mathbf{1}\left(T_{n_{w}^{-1} \cdot \lambda_{R^{\prime}}^{+}}^{Q}\right)=q_{n_{w}^{-1} \cdot \lambda_{R^{\prime}}^{+}, Q}$. Therefore the action of $T_{\lambda_{R^{\prime}}^{+}}$on this subquotient 
is given by a power of $p$ and it is 1 if and only if $q\left(Q, n_{w}^{-1} \cdot \lambda_{R^{\prime}}^{+}\right)=1$ and $\ell_{Q}\left(n_{w}^{-1} \cdot \lambda_{R^{\prime}}^{+}\right)=0$.

Put $Q^{\prime}=n_{w_{G} w_{Q}} Q^{\mathrm{op}} n_{w_{G} w_{Q}}^{-1}$. Note that $n_{w}^{-1} \cdot \lambda_{R^{\prime}}^{+}$is $Q$-negative if and only if $\left(n_{w_{G} w_{Q}} n_{w}^{-1}\right) \cdot \lambda_{R^{\prime}}^{+}$is $Q^{\prime}$-positive since $\left(w_{G} w_{Q}\right)^{-1}\left(\Sigma^{+} \backslash \Sigma_{Q^{\prime}}^{+}\right)=\Sigma^{-} \backslash$ $\Sigma_{Q}^{-}$. Therefore $n_{w}^{-1} \cdot \lambda_{R^{\prime}}^{+}$is $Q$-negative if and only if $w_{G} w_{Q} w^{-1} \in W_{0, R^{\prime}}$ by Lemma 5.3, namely $w \in W_{0, R^{\prime}} w_{G} w_{Q}$.

The length of $n_{w}^{-1} \cdot \lambda_{R^{\prime}}^{+} \in W_{Q}(1)$ is 0 if and only if $\left\langle\alpha, \nu\left(n_{w}^{-1} \cdot \lambda_{R^{\prime}}^{+}\right)\right\rangle=0$ for any $\alpha \in \Sigma_{Q}$. Since $\left\langle\beta, \nu\left(\lambda_{R^{\prime}}^{+}\right)\right\rangle=0$ if and only if $\beta \in \Sigma_{R^{\prime}}$, the length of $\lambda_{R^{\prime}}^{+} \in$ $W_{Q}(1)$ is 0 if and only if $w\left(\Sigma_{Q}\right) \subset \Sigma_{R^{\prime}}$. Since we have $w \in W_{0, R^{\prime}} w_{G} w_{Q}$, $w\left(\Sigma_{Q}\right) \subset \Sigma_{R^{\prime}}$ if and only if $w_{G}\left(\Sigma_{Q}\right) \subset \Sigma_{R^{\prime}}$.

The subset $W_{0, R^{\prime}} \cap Q^{Q^{\prime}} W_{0}$ is closed in ${ }^{Q^{\prime}} W_{0}$. Hence by Proposition 2.21, $W_{0, R^{\prime}} w_{G} w_{Q} \cap W_{0}^{Q}$ is open in $W_{0}^{Q}$. Therefore the exactness of (5.5) follows from the following general lemma. In the following lemma, we call a subset $A$ of a partially ordered set open if $a \in A$ and $b \geq a$ implies $b \in A$.

Lemma 5.15. Let $\Gamma$ be a partially ordered set and $M$ a $C[X]$-module with the decomposition into $C$-submodules $M=\bigoplus_{\gamma \in \Gamma} M_{\gamma}$. Assume the following.

- For each open subset $\Delta \subset \Gamma, M_{\Delta}=\bigoplus_{\gamma \in \Delta} M_{\gamma}$ is $C[X]$-stable.

- For each open subset $\Delta \subset \Gamma$ and a minimal element $\gamma \in \Delta$, the action of $X$ on $M_{\Delta} / M_{\Delta \backslash\{\gamma\}}$ is given by $p^{n_{\gamma}}$ for some $n_{\gamma} \in \mathbb{Z}_{\geq 0}$.

- The subset $\Gamma_{0}=\left\{\gamma \in \Gamma_{0} \mid n_{\gamma}=0\right\}$ is open.

Then for each open subset $\Delta \subset \Gamma$ and a minimal element $\gamma \in \Delta$, the homomorphism

$$
\operatorname{Hom}_{C[X]}\left(C\left[X^{ \pm 1}\right], M_{\Delta}\right) \rightarrow \operatorname{Hom}_{C[X]}\left(C\left[X^{ \pm 1}\right], M_{\Delta} / M_{\Delta \backslash\{\gamma\}}\right)
$$

is surjective.

Proof. Put $\Delta_{0}=\Delta \cap \Gamma_{0}$. We divide the map into two maps:

$$
\begin{gathered}
\operatorname{Hom}_{C[X]}\left(C\left[X^{ \pm 1}\right], M_{\Delta}\right) \rightarrow \operatorname{Hom}_{C[X]}\left(C\left[X^{ \pm 1}\right], M_{\Delta} / M_{\Delta_{0} \backslash\{\gamma\}}\right) \\
\operatorname{Hom}_{C[X]}\left(C\left[X^{ \pm 1}\right], M_{\Delta} / M_{\Delta_{0} \backslash\{\gamma\}}\right) \rightarrow \operatorname{Hom}_{C[X]}\left(C\left[X^{ \pm 1}\right], M_{\Delta} / M_{\Delta \backslash\{\gamma\}}\right) .
\end{gathered}
$$

We prove that both maps are surjective.

(1) We prove the surjectivity of (5.7). Since $M_{\Delta_{0} \backslash\{\gamma\}}$ have a filtration such that $X$ in invertible on successive quotients, $X$ is also invertible on $M_{\Delta_{0} \backslash\{\gamma\}}$. Hence the claim follows from the following claim.

Claim. Let $N$ be a $C[X]$-module and assume that $X$ is invertible on $N$. Then $\operatorname{Ext}_{C[X]}^{1}\left(C\left[X^{ \pm 1}\right], N\right)=0$.

Proof of Claim. Let $0 \rightarrow N \rightarrow L \rightarrow C\left[X^{ \pm 1}\right] \rightarrow 0$ be an exact sequence of $C[X]$-modules. Since $X$ is invertible on $N$ and $C\left[X^{ \pm 1}\right], X$ is also invertible on $L$. Namely $0 \rightarrow N \rightarrow L \rightarrow C\left[X^{ \pm 1}\right] \rightarrow 0$ is also an exact sequence of $C\left[X^{ \pm 1}\right]$-modules. Hence $\operatorname{Ext}_{C[X]}^{1}\left(C\left[X^{ \pm 1}\right], N\right)=\operatorname{Ext}_{C\left[X^{ \pm 1}\right]}^{1}\left(C\left[X^{ \pm 1}\right], N\right)$. This is obviously zero. 
(2) Next we prove the surjectivity of (5.8) assuming $\gamma \in \Gamma_{0}$. We have $M_{\Delta} / M_{\Delta_{0} \backslash\{\gamma\}} \supset M_{\Delta_{0}} / M_{\Delta_{0} \backslash\{\gamma\}} \simeq M_{\gamma} \simeq M_{\Delta} / M_{\Delta \backslash\{\gamma\}}$. Hence from the following diagram

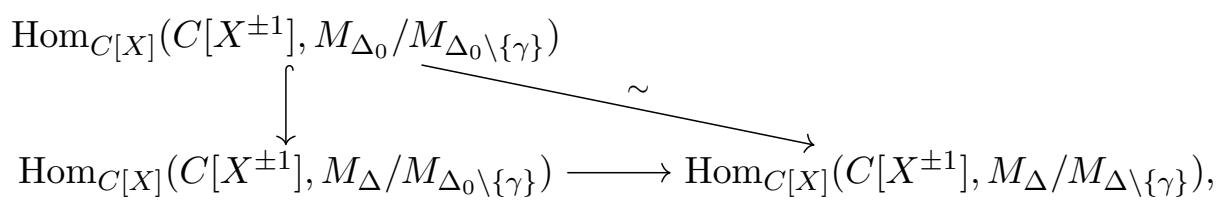

(5.8) is surjective in this case.

(3) Finally we prove the surjectivity of (5.8) assuming $\gamma \notin \Gamma_{0}$. Note that in general we have $\operatorname{Hom}_{C[X]}\left(C\left[X^{ \pm 1}\right], N\right)=\left\{\left(m_{n}\right)_{n \in \mathbb{Z}_{\geq 0}} \mid m_{n} \in N, X m_{n}=\right.$ $\left.m_{n-1}\right\}$ by $\varphi \mapsto\left(\varphi\left(X^{-n}\right)\right)$. Recall that the action of $X$ on $M_{\Delta} / M_{\Delta \backslash\{\gamma\}}$ is given by $p^{n_{\gamma}}$ with $n_{\gamma}>0$. (We have assumed that $\gamma \notin \Gamma_{0}$.) Hence to give an element in $\operatorname{Hom}_{C[X]}\left(C\left[X^{ \pm 1}\right], M_{\Delta} / M_{\Delta \backslash\{\gamma\}}\right)$ is equivalent to give a sequence of elements $\left(m_{n}^{(\gamma)}\right)$ in $M_{\gamma}$ such that $p^{n_{\gamma}} m_{n}^{(\gamma)}=m_{n-1}^{(\gamma)}$. We prove that we can extend this element to $m_{n}=\left(m_{n}^{(\delta)}\right)_{\delta \in \Delta, n \in \mathbb{Z}_{\geq 0}}$ in $M_{\Delta}=\bigoplus_{\delta \in \Delta} M_{\delta}$ such that $X m_{n}=m_{n-1}$. Since $p^{n_{\gamma}} m_{n}^{(\gamma)}=m_{n-1}^{(\gamma)}$ with $n_{\gamma}>0$, we have $m_{n}^{(\gamma)} \in \bigcap_{k} p^{k} M_{\gamma}$. We prove that we can take an extension $m_{n}=\left(m_{n}^{(\delta)}\right)$ from $\bigcap_{k} p^{k} M_{\Delta}$.

According to the decomposition $M=\bigoplus_{\gamma \in \Gamma} M_{\gamma}$, we have a linear map $X_{\gamma_{1}, \gamma_{2}} \in \operatorname{Hom}_{C}\left(M_{\gamma_{2}}, M_{\gamma_{1}}\right)$ such that $X m_{\gamma}=\sum_{\gamma^{\prime} \in \Gamma} X_{\gamma^{\prime}, \gamma} m_{\gamma}$ for $m_{\gamma} \in M_{\gamma} \subset$ $M$. By the assumption $X_{\gamma_{1}, \gamma_{2}}=0$ if $\gamma_{1} \nsupseteq \gamma_{2}$. We also have $X_{\gamma^{\prime}, \gamma^{\prime}}=p^{n_{\gamma^{\prime}}}$ with $n_{\gamma^{\prime}} \geq 0$. The condition $X m_{n}=m_{n-1}$ is equivalent to

$$
m_{n-1}^{\left(\delta_{1}\right)}=\sum_{\delta_{2} \leq \delta_{1}} X_{\delta_{1}, \delta_{2}} m_{n}^{\left(\delta_{2}\right)}
$$

We prove the existence of $m_{n}=\left(m_{n}^{(\delta)}\right)$ using a triangular argument, namely we take $m_{n}^{(\delta)}$ which satisfies (5.9) inductively on $\delta$.

Let $\delta \in \Delta \backslash\{\gamma\}$ and assume that we have taken $m_{n}^{\left(\delta^{\prime}\right)}$ for $\delta^{\prime} \in \Delta$ such that $\delta^{\prime}<\delta$. Since $m_{n}^{\left(\delta^{\prime}\right)} \in \bigcap_{k} p^{k} M_{\delta^{\prime}}$ for any $\delta^{\prime}<\delta$, we can take $x_{n}^{\left(\delta^{\prime}\right)} \in$ $\bigcap_{k} p^{k} M_{\delta^{\prime}}$ such that $p^{n_{\delta}} x_{n}^{\left(\delta^{\prime}\right)}=m_{n}^{\left(\delta^{\prime}\right)}$. We also have $x_{n-1}^{(\delta)} \in \bigcap_{k} p^{k} M_{\delta}$ such that $p^{n_{\delta}} x_{n-1}^{(\delta)}=m_{n-1}^{(\delta)}$. Then define $m_{n}^{(\delta)}=x_{n-1}^{(\delta)}-\sum_{\delta^{\prime}<\delta} X_{\delta, \delta^{\prime}} x_{n}^{\left(\delta^{\prime}\right)}$ and it satisfies $m_{n-1}^{(\delta)}=p^{n_{\delta}} m_{n}^{(\delta)}+\sum_{\delta^{\prime}<\delta} X_{\delta, \delta^{\prime}} m_{n}^{\left(\delta^{\prime}\right)}$. Since $X_{\delta, \delta}=p^{n_{\delta}}$, it means that (5.9) holds for $\delta_{1}=\delta$.

For (5.6), we use the following lemma with Lemma [5.15,

Lemma 5.16. Set $B=W_{0}^{Q} \backslash \bigcup_{Q_{1} \supseteq Q} W_{0}^{Q_{1}}$. For $w \in B$, we define $\operatorname{St}_{Q, w} \subset$ $\mathrm{St}_{Q}(\mathbf{1})$ by the image of

$$
\left\{\varphi \in I_{Q}(\mathbf{1}) \mid \varphi\left(T_{n_{v}}\right)=0\left(v \in W_{0}^{Q} \backslash\{w\}\right)\right\} \hookrightarrow I_{Q}(\mathbf{1}) \rightarrow \operatorname{St}_{Q}(\mathbf{1}) .
$$

Then we have $\operatorname{St}_{Q}(\mathbf{1})=\bigoplus_{w \in B} \operatorname{St}_{Q, w}$ and for any open $A \subset W_{0}^{Q}$, we have $\bigoplus_{w \in A \cap B} \mathrm{St}_{Q, w}=\mathrm{St}_{Q, A}$.

Proof. We prove $\sum_{v \in A \cap B} \mathrm{St}_{Q, v}=\mathrm{St}_{Q, A}$ by induction on $\# A$. Let $w \in A$ be a minimal element and set $A^{\prime}=A \backslash\{w\}$. If $w \notin B$ then $A \cap B=A^{\prime} \cap B$ and 
$\mathrm{St}_{Q, A}=\mathrm{St}_{Q, A^{\prime}}$ by Lemma 3.11, If $w \in B$, then $\mathrm{St}_{Q, A} / \mathrm{St}_{Q, A^{\prime}} \simeq I_{Q, A} / I_{Q, A^{\prime}} \simeq$ $\left\{\varphi \in I_{Q}(\mathbf{1}) \mid \varphi\left(T_{n_{v}}\right)=0\left(v \in W_{0}^{Q} \backslash\{w\}\right)\right\}$. Therefore $\mathrm{St}_{Q, A^{\prime}}+\mathrm{St}_{Q, w}=\mathrm{St}_{Q, A}$. By inductive hypothesis, $\mathrm{St}_{Q, A^{\prime}}=\sum_{v \in A^{\prime} \cap B} \mathrm{St}_{Q, v}$. We get $\sum_{v \in A \cap B} \mathrm{St}_{Q, v}=$ $\mathrm{St}_{Q, A}$.

Let $x_{w} \in \operatorname{St}_{Q, w}(w \in B)$ such that $\sum_{w \in B} x_{w}=0$. Assume that there exists $w \in B$ such that $x_{w} \neq 0$ and assume that $w$ is minimal subject to $x_{w} \neq$ 0 . Put $A^{\prime}=\left\{v \in W_{0}^{Q} \mid v \geq v_{1}\right.$ for some $v_{1} \in W_{0}^{Q} \backslash\{w\}$ such that $\left.x_{v_{1}} \neq 0\right\}$. Then $A^{\prime}$ is open and $w \notin A^{\prime}$. Hence $\sum_{v \neq w} x_{v} \in \operatorname{St}_{Q, A^{\prime}}$. Set $A=A^{\prime} \cup\{w\}$. Let $y_{w} \in I_{Q}\left(e_{Q}(\sigma)\right)$ such that $y_{w}\left(T_{n_{v}}\right)=0$ for $v \in W_{0}^{Q} \backslash\{w\}$ and the image of $y_{w}$ in $\mathrm{St}_{Q, w}$ is $x_{w}$. Then the image of $y_{w}$ under

$$
\left\{\varphi \in I_{Q}(\mathbf{1}) \mid \varphi\left(T_{n_{v}}\right)=0\left(v \in W_{0}^{Q} \backslash\{w\}\right)\right\} \simeq I_{Q, A} / I_{Q, A^{\prime}} \simeq \mathrm{St}_{Q, A} / \mathrm{St}_{Q, A^{\prime}}
$$

is equal to the image of $x_{w}$. Here the last isomorphism is by Lemma 3.11. Since $\sum_{v \neq w} x_{v} \in \mathrm{St}_{Q, A^{\prime}}$, it is equal to the image of $\sum_{v \in B} x_{v}$ by $\operatorname{St}_{Q, A} \rightarrow$ $\mathrm{St}_{Q, A} / \mathrm{St}_{Q, A^{\prime}}$. Since $\sum_{v \in B} x_{v}=0$, we have $y_{w}=0$. This contradicts to $x_{w} \neq 0$.

5.5. $R_{P}$ and Steinberg modules. As in the previous subsection, let $P$ be a parabolic subgroup and $\sigma$ an $\mathcal{H}_{P}$-module which has the extension to $\mathcal{H}$. We prove Proposition 5.11 in this subsection. As in the case of $L_{R}$, we start with the following lemma.

Lemma 5.17. Assume that $\bigcap_{n \in \mathbb{Z}_{>0}} p^{n} \sigma=0$. We have

$$
R_{R}\left(e_{G}(\sigma)\right)= \begin{cases}e_{R}\left(R_{R \cap P}^{P}(\sigma)\right) & \left(\Delta=\Delta_{R} \cup \Delta_{P}\right), \\ 0 & (\text { otherwise }) .\end{cases}
$$

Proof. First assume that $\Delta \neq \Delta_{R} \cup \Delta_{P}$ and we prove $R_{R}\left(e_{G}(\sigma)\right)=0$. Let $R_{1}$ be a parabolic subgroup corresponding to $\Delta_{R} \cup \Delta_{P}$. It is sufficient to prove that $R_{R_{1}}\left(e_{G}(\sigma)\right)=0$. Take $\lambda_{R_{1}^{\prime}}^{+}$as in Proposition 2.5 for $R_{1}^{\prime}$ where $R_{1}^{\prime}=n_{w_{G} w_{R_{1}}} R_{1}^{\mathrm{op}} n_{w_{G} w_{R_{1}}}^{-1}$. Let $P_{2}$ be a parabolic subgroup corresponding to $\Delta \backslash \Delta_{P}$. Since $R_{1}^{\prime} \neq G$ and $P \subset R_{1}^{\prime}$, there exists $\alpha \in \Delta \backslash \Delta_{R_{1}^{\prime}}=\Delta_{P_{2}} \backslash \Delta_{R_{1}^{\prime}}$ and for such $\alpha$, we have $\left\langle\alpha, \nu\left(\lambda_{R_{1}^{\prime}}^{+}\right)\right\rangle<0$. Hence the length of $\lambda_{R_{1}^{\prime}}^{+}$as an element of $W_{P_{2}}(1)$ is positive. Therefore $e_{G}(\sigma)\left(T_{\lambda_{R_{1}^{\prime}}^{+}}\right)$is divided by $p$. Hence, the image of any $\varphi \in \operatorname{Hom}_{\left(\mathcal{H}_{R_{1}^{\prime}}^{+}, j_{R_{1}^{\prime}}^{+}\right)}\left(\mathcal{H}_{R_{1}^{\prime}}, e_{G}(\sigma)\right)=\operatorname{Hom}_{C\left[T_{\lambda_{R_{1}^{\prime}}}\right]}\left(C\left[\left(T_{\lambda_{R_{1}^{+}}^{+}}\right)^{ \pm 1}\right], e_{G}(\sigma)\right)$ is in $\bigcap_{n \in \mathbb{Z}_{>0}} p^{n} e_{G}(\sigma)=0$. (As $C$-modules, we have $e_{G}(\sigma)=\sigma$.) We get $R_{R_{1}}\left(e_{G}(\sigma)\right)=0$.

Now assume that $\Delta_{R} \cup \Delta_{P}=\Delta$, or in other words, $\Delta_{P_{2}} \subset \Delta_{R}$. Since $\Delta=\Delta_{P} \cup \Delta_{P_{2}}$ and $\Delta_{R}=\Delta_{R \cap P} \cup \Delta_{P_{2}}$ are orthogonal decompositions, we have decompositions $W_{0}=W_{0, P} \times W_{0, P_{2}}$ and $W_{0, R}=W_{0, P \cap R} \times W_{0, P_{2}}$ as Coxeter groups. Hence we have $w_{G}=w_{P} w_{P_{2}}$ and $w_{R}=w_{P \cap R} w_{P_{2}}$. We have $w_{G} w_{R}=w_{P} w_{P \cap R}$. Set $R^{\prime}=n_{w_{G} w_{R}} R^{\mathrm{op}} n_{w_{G} w_{R}}^{-1}$. We also have $P \cap R^{\prime}=n_{w_{P} w_{P \cap R}}(P \cap R)^{\mathrm{op}} n_{w_{P} w_{P \cap R}}^{-1}$. By the definition of the right adjoint functors, we have

$$
\begin{aligned}
R_{R}\left(e_{G}(\sigma)\right) & =n_{w_{G} w_{R}}^{-1} \operatorname{Hom}_{\left(\mathcal{H}_{R^{\prime}}^{+}, j_{R^{\prime}}^{+}\right)}\left(\mathcal{H}_{R^{\prime}}, e_{G}(\sigma)\right), \\
R_{P \cap R}^{P}(\sigma) & =n_{w_{P} w_{P \cap R}}^{-1} \operatorname{Hom}_{\left(\mathcal{H}_{P \cap R^{\prime}}^{P+}, j_{P \cap R^{\prime}}^{P+}\right.}\left(\mathcal{H}_{P \cap R^{\prime}}, \sigma\right)
\end{aligned}
$$


Since $w_{G} w_{R}=w_{P} w_{P \cap R}$ from the assumption, replacing $R^{\prime}$ with $R$, with Lemma 2.27, it is sufficient to prove that $A=\operatorname{Hom}_{\left(\mathcal{H}_{R}^{+}, j_{R}^{+}\right)}\left(\mathcal{H}_{R}, e_{G}(\sigma)\right)$ is isomorphic to $e_{R}(B)$ where $B=\operatorname{Hom}_{\left(\mathcal{H}_{P \cap R}^{P+}, j_{P \cap R}^{P+}\right)}\left(\mathcal{H}_{P \cap R}, \sigma\right)$.

The map $\varphi \mapsto\left(\varphi\left(\left(T_{\lambda_{R}^{+}}^{R}\right)^{-n}\right)\right)$ gives an isomorphism

$$
A \simeq\left\{\left(x_{n}\right)_{n \in \mathbb{Z}_{\geq 0}} \mid x_{n+1} e_{G}(\sigma)\left(T_{\lambda_{R}^{+}}\right)=x_{n}, x_{n} \in e_{G}(\sigma)\right\}
$$

Since $\lambda_{R}^{+}$is anti-dominant, $T_{\lambda_{R}^{+}}=E_{o_{-}}\left(\lambda_{R}^{+}\right)$and $T_{\lambda_{R}^{+}}^{P}=E_{o_{-, P}}^{P}\left(\lambda_{R}^{+}\right)$by (2.3). Hence we have $T_{\lambda_{R}^{+}}=E_{o_{-}}\left(\lambda_{R}^{+}\right)=j_{P}^{-*}\left(E_{o_{-, P}}^{P}\left(\lambda_{R}^{+}\right)\right)=j_{P}^{-*}\left(T_{\lambda_{R}^{+}}^{P}\right)$ by Proposition 2.6. Therefore $e_{G}(\sigma)\left(T_{\lambda_{R}^{+}}\right)=\sigma\left(T_{\lambda_{R}^{+}}^{P}\right)$. Hence we have

$$
A \simeq\left\{\left(x_{n}\right)_{n \in \mathbb{Z}_{\geq 0}} \mid x_{n+1} \sigma\left(T_{\lambda_{R}^{+}}^{P}\right)=x_{n}, x_{n} \in \sigma\right\}
$$

Since $R \supset P_{2}$, we have $\Sigma^{+} \backslash \Sigma_{R}^{+}=\Sigma_{P}^{+} \backslash \Sigma_{P \cap R}^{+}$. Therefore we can take $\lambda_{R}^{+}$as $\lambda_{R \cap P}^{P+}$. Hence

$$
\left\{\left(x_{n}\right)_{n \in \mathbb{Z}_{\geq 0}} \mid x_{n+1} \sigma\left(T_{\lambda_{R}^{+}}^{P}\right)=x_{n}\right\} \simeq B .
$$

Namely there exists an isomorphism $A \simeq B$ as vector spaces which is characterized by $\varphi\left(\left(T_{\lambda_{R}^{+}}^{R}\right)^{-n}\right)=\psi\left(\left(T_{\lambda_{R}^{+}}^{P \cap R}\right)^{-n}\right)$ for any $n \in \mathbb{Z}_{\geq 0}$ where $\varphi \in A$ corresponds to $\psi \in B$ by this isomorphism.

We prove that this isomorphism is $\left(\mathcal{H}_{P \cap R}^{R-}, j_{P \cap R}^{R-*}\right)$-equivariant. Let $w \in$ $W_{P \cap R}^{R-}(1)$. By the assumption $\Delta_{P_{2}} \subset \Delta_{R}, \Sigma_{R}^{+} \backslash \Sigma_{P \cap R}^{+}=\Sigma_{P_{2}}^{+}=\Sigma^{+} \backslash \Sigma_{P}^{+}$. Hence $w \in W_{P}^{-}(1)$. Take $k \in \mathbb{Z}_{\geq 0}$ such that $w\left(\lambda_{R}^{+}\right)^{k}$ is $R$-positive. Since $\varphi$ is $\left(\mathcal{H}_{R}^{+}, j_{R}^{+}\right)$-equivariant, using Lemma 2.6, we have

$$
\begin{aligned}
\left(\varphi j_{P \cap R}^{R-*}\left(E_{o_{-, P \cap R}}^{P \cap R}(w)\right)\right)\left(\left(T_{\lambda_{R}^{+}}^{R}\right)^{-n}\right) & =\varphi\left(E_{o_{-, R}}^{R}(w)\left(T_{\lambda_{R}^{+}}^{R}\right)^{-n}\right) \\
& =\varphi\left(E_{o_{-, R}}^{R}\left(w\left(\lambda_{R}^{+}\right)^{k}\right)\left(T_{\lambda_{R}^{+}}^{R}\right)^{-(n+k)}\right) \\
& =\varphi\left(\left(T_{\lambda_{R}^{+}}^{R}\right)^{-(n+k)} j_{R}^{+}\left(E_{o_{-}}\left(w\left(\lambda_{R}^{+}\right)^{k}\right)\right)\right) \\
& =\varphi\left(\left(T_{\lambda_{R}^{+}}^{R}\right)^{-(n+k)}\right) e_{G}(\sigma)\left(E_{o_{-}}\left(w\left(\lambda_{R}^{+}\right)^{k}\right)\right) .
\end{aligned}
$$

Since $\lambda_{R}^{+}$is in the center of $W_{R}(1)$ and $P_{2} \subset R, \lambda_{R}^{+}$is also in the center of $W_{P_{2}}(1)$, hence we have $\left\langle\alpha, \nu\left(\lambda_{R}^{+}\right)\right\rangle=0$ for any $\alpha \in \Delta_{P_{2}}$. Hence for any $\alpha \in \Sigma^{+} \backslash \Sigma_{P}^{+}$, we have $\left\langle\alpha, \nu\left(\lambda_{R}^{+}\right)\right\rangle=0$ since $\Sigma^{+} \backslash \Sigma_{P}^{+}=\Sigma_{P_{2}}^{+}$. Therefore $\lambda_{R}^{+}$is both $P$-positive and $P$-negative. Recall that $w$ is also $P$-negative. Therefore $w\left(\lambda_{R}^{+}\right)^{k}$ is also $P$-negative. Hence $e_{G}(\sigma)\left(E_{o_{-}}\left(w\left(\lambda_{R}^{+}\right)^{k}\right)\right)=\sigma\left(E_{o_{-, P}}^{P}\left(w\left(\lambda_{R}^{+}\right)^{k}\right)\right)$ by the definition of the extension and Lemma 2.6. Therefore

$$
\begin{aligned}
\varphi\left(\left(T_{\lambda_{R}^{+}}^{R}\right)^{-(n+k)}\right) e_{G}(\sigma)\left(E_{o_{-}}\left(w\left(\lambda_{R}^{+}\right)^{k}\right)\right) & =\varphi\left(\left(T_{\lambda_{R}^{+}}^{R}\right)^{-(n+k)}\right) \sigma\left(E_{o_{-, P}}^{P}\left(w\left(\lambda_{R}^{+}\right)^{k}\right)\right) \\
& =\psi\left(\left(T_{\lambda_{R}^{+}}^{P \cap R}\right)^{-(n+k)}\right) \sigma\left(E_{o_{-}, P}^{P}\left(w\left(\lambda_{R}^{+}\right)^{k}\right)\right) .
\end{aligned}
$$

Since $w\left(\lambda_{R}^{+}\right)^{k} \in W_{P \cap R}(1)$ is $R$-positive, we have $w\left(\lambda_{R}^{+}\right)^{k} \in W_{P \cap R}^{P+}(1)$. Therefore $E_{o_{-, P}}^{P}\left(w\left(\lambda_{R}^{+}\right)^{k}\right)=j_{P \cap R}^{P+}\left(E_{o_{-}, P \cap R}^{P \cap R}\left(w\left(\lambda_{R}^{+}\right)^{k}\right)\right)$ by Lemma 2.6. Hence

$$
\begin{aligned}
\psi\left(\left(T_{\lambda_{R}^{+}}^{P \cap R}\right)^{-(n+k)}\right) \sigma\left(E_{o_{-}, P}^{P}\left(w\left(\lambda_{R}^{+}\right)^{k}\right)\right) & =\psi\left(\left(T_{\lambda_{R}^{+}}^{P \cap R}\right)^{-(n+k)} E_{o_{-}, P \cap R}^{P \cap R}\left(w\left(\lambda_{R}^{+}\right)^{k}\right)\right) \\
& =\left(\psi E_{o_{-, P \cap R}}^{P \cap R}(w)\right)\left(\left(T_{\lambda_{R}^{+}}^{P \cap R}\right)^{-n}\right) .
\end{aligned}
$$


Hence $A \simeq B$ as $\left(\mathcal{H}_{P \cap R}^{R-}, j_{P \cap R}^{R-*}\right)$-modules.

Let $w \in W_{P_{2} \cap R \text {,aff }}(1)$. Then $w \in W_{P_{2} \text {,aff }}(1)$. Since $\Sigma^{+} \backslash \Sigma_{R}^{+} \subset \Sigma^{+} \backslash \Sigma_{P_{2}}^{+}=$ $\Sigma_{P}^{+}$and for any $\alpha \in \Sigma_{P}^{+}$is orthogonal to elements in $\nu\left(\Lambda(1) \cap W_{P_{2} \text {,aff }}(1)\right)$, $w$ is both $R$-positive and $R$-negative. Hence by Corollary 2.7, we have $j_{R}^{+}\left(T_{w}^{R *}\right)=T_{w}^{*}$. Therefore, for $\varphi \in A$, we have

$$
\begin{aligned}
\left(\varphi T_{w}^{R *}\right)\left(\left(T_{\lambda_{R}^{+}}^{R}\right)^{-n}\right) & =\varphi\left(T_{w}^{R *}\left(T_{\lambda_{R}^{+}}^{R}\right)^{-n}\right) \\
& =\varphi\left(\left(T_{\lambda_{R}^{+}}^{R}\right)^{-n} T_{w}^{R *}\right) \\
& =\varphi\left(\left(T_{\lambda_{R}^{+}}^{R}\right)^{-n}\right) e_{G}(\sigma)\left(j_{R}^{+}\left(T_{w}^{R *}\right)\right) \\
& =\varphi\left(\left(T_{\lambda_{R}^{+}}^{R}\right)^{-n}\right) e_{G}(\sigma)\left(T_{w}^{*}\right) \\
& =\varphi\left(\left(T_{\lambda_{R}^{+}}^{R}\right)^{-n}\right) .
\end{aligned}
$$

Therefore $T_{w}^{R *}=1$ on $A$. Hence $A \simeq e_{R}(B)$ by the definition of the extension.

Proof of Proposition 5.11. By Lemma 5.12, we have

$$
\bigoplus_{Q_{1} \supsetneq Q} R_{R}\left(I_{Q_{1}}\left(e_{Q_{1}}(\sigma)\right)\right) \rightarrow R_{R}\left(I_{Q}\left(e_{Q}(\sigma)\right)\right) \rightarrow R_{R}\left(\operatorname{St}_{Q}(\sigma)\right) \rightarrow 0
$$

By Proposition 5.1 ,

$$
\bigoplus_{Q^{\prime} \supsetneq Q} I_{Q^{\prime} \cap R}^{R}\left(R_{Q^{\prime} \cap R}^{Q^{\prime}}\left(e_{Q^{\prime}}(\sigma)\right)\right) \rightarrow I_{Q \cap R}^{R}\left(R_{Q \cap R}^{Q}\left(e_{Q}(\sigma)\right)\right) \rightarrow R_{R}\left(\operatorname{St}_{Q}(\sigma)\right) \rightarrow 0 .
$$

If $\Delta_{Q} \neq \Delta_{Q \cap R} \cup \Delta_{P}$, then $R_{Q \cap R}^{Q}\left(e_{Q}(\sigma)\right)=0$ by Lemma [5.17. Hence $R_{R}\left(\operatorname{St}_{Q}(\sigma)\right)=0$. Assume that $\Delta_{Q}=\Delta_{Q \cap R} \cup \Delta_{P}$. Then $R_{Q \cap R}^{Q}\left(e_{Q}(\sigma)\right)=$ $e_{Q \cap R}\left(R_{P \cap R}^{P}(\sigma)\right)$. Let $Q^{\prime} \supsetneq Q$. If $\Delta_{Q^{\prime}}=\Delta_{Q^{\prime} \cap R} \cup \Delta_{P}$, then $R_{Q^{\prime} \cap R}^{Q^{\prime}}\left(e_{Q^{\prime}}(\sigma)\right)=$ $e_{Q^{\prime} \cap R}\left(R_{P \cap R}^{P}(\sigma)\right)$ and $Q^{\prime} \cap R \supsetneq Q \cap R$. Otherwise, it is zero. Putting $Q^{\prime \prime}=Q^{\prime} \cap R$, we have

$$
\begin{aligned}
\bigoplus_{R \supset Q^{\prime \prime} \supsetneq Q \cap R} I_{Q^{\prime \prime}}^{R}\left(e_{Q^{\prime \prime}}\left(R_{P \cap R}^{R}(\sigma)\right)\right) \rightarrow I_{Q \cap R}^{R}\left(e_{Q \cap R}\left(R_{P \cap R}^{P}(\sigma)\right)\right) & \\
& \rightarrow R_{R}\left(\operatorname{St}_{Q}(\sigma)\right) \rightarrow 0 .
\end{aligned}
$$

Therefore we have $R_{R}\left(\operatorname{St}_{Q}(\sigma)\right)=\operatorname{St}_{Q \cap R}^{R}\left(R_{P \cap R}^{P}(\sigma)\right)$.

5.6. Supersingular modules. Assume that $p=0$ in $C$.

Proposition 5.18. Let $P \subsetneq G$ be a proper parabolic subgroup and $\pi a$ supersingular $\mathcal{H}$-module. Then we have $L_{P}(\pi)=R_{P}(\pi)=0$.

We need a lemma.

Lemma 5.19. Assume that $\lambda \in Z(\Lambda(1))$ satisfies that for any $w \in W_{0}$, we have $n_{w} \cdot \lambda=\lambda$ if and only if $w(\nu(\lambda))=\nu(\lambda)$. Put $\mathcal{O}=W(1) \cdot \lambda$. Then for any orientation o and $n \in \mathbb{Z}_{\geq 0}$, we have $z_{\mathcal{O}}^{n} E_{o}(\lambda)=E_{o}(\lambda)^{n+1}$. 
Proof. Since $\lambda$ is in the center of $\Lambda(1)$, we have $\mathcal{O}=\left\{n_{w} \cdot \lambda \mid w \in W_{0}\right\}$. We also have $\mathcal{O}=\left\{n_{w} \cdot \lambda \mid w \in W_{0} / \operatorname{Stab}_{W_{0}}(\nu(\lambda))\right\}$ by the condition on $\lambda$. Therefore we have $z_{\mathcal{O}}=\sum_{w \in W_{0} / \operatorname{Stab}_{W_{0}} \nu(\lambda)} E_{o}\left(n_{w} \cdot \lambda\right)$. Hence

$$
z_{\mathcal{O}}^{2}=\sum_{v_{1}, v_{2} \in W_{0} / \operatorname{Stab}_{W_{0}} \nu(\lambda)} E_{o}\left(n_{v_{1}} \cdot \lambda\right) E_{o}\left(n_{v_{2}} \cdot \lambda\right) .
$$

If $v_{1}, v_{2} \in W_{0}$ does not belong to the same coset in $W_{0} / \operatorname{Stab}_{W_{0}} \nu(\lambda)$, then $v_{1}(\nu(\lambda))$ and $v_{2}(\nu(\lambda))$ are not in the same closed chamber. Hence $E_{o}\left(n_{v_{1}}\right.$. $\lambda) E_{o}\left(n_{v_{2}} \cdot \lambda\right)=0$ by (2.1) and Lemma 2.11, Therefore

$$
z_{\mathcal{O}}^{2}=\sum_{v \in W_{0} / \operatorname{Stab}_{W_{0}} \nu(\lambda)} E_{o}\left(n_{v} \cdot \lambda\right)^{2} .
$$

Inductively, we get

$$
z_{\mathcal{O}}^{n}=\sum_{v \in W_{0} / \operatorname{Stab}_{W_{0}} \nu(\lambda)} E_{o}\left(n_{v} \cdot \lambda\right)^{n}
$$

Hence

$$
z_{\mathcal{O}}^{n} E_{o}(\lambda)=\sum_{v \in W_{0} / \operatorname{Stab}_{W_{0}} \nu(\lambda)} E_{o}\left(n_{v} \cdot \lambda\right)^{n} E_{o}(\lambda)
$$

If $v \notin \operatorname{Stab}_{W_{0}} \nu(\lambda)$, then $E_{o}\left(n_{v} \cdot \lambda\right) E_{o}(\lambda)=0$. Hence

$$
z_{\mathcal{O}}^{n} E_{o}(\lambda)=E_{o}(\lambda)^{n+1}
$$

We get the lemma.

Proof of Proposition [5.18. Let $\lambda=\lambda_{P}^{-} \in Z\left(W_{P}(1)\right)$ be as in Proposition 2.5. If $w \in W_{0}$ fixes $\nu(\lambda)$, then $w \in W_{0, P}$. Since $\lambda \in Z\left(W_{P}(1)\right)$, we have $n_{w} \cdot \lambda=\lambda$. Namely, $\lambda$ satisfies the condition of the above lemma. We also have $L_{P}(\pi)=\pi E_{o_{-}}(\lambda)^{-1}$.

Set $\mathcal{O}=W(1) \cdot \lambda$. Let $x \in \pi$. Since $\pi$ is supersingular, there exists $n \in \mathbb{Z}_{>0}$ such that $x z_{\mathcal{O}}^{n}=0$. Hence $x z_{\mathcal{O}}^{n} E_{o_{-}}(\lambda)=0$. Therefore we have $x E_{o_{-}}(\lambda)^{n+1}=0$ by the above lemma. Hence the image of $x$ in $L_{P}(\pi)=$ $\pi E_{o_{-}}(\lambda)^{-1}$ is zero. We get $L_{P}(\pi)=0$.

Next we prove $R_{P}(\pi)=n_{w_{G} w_{P}}^{-1} \operatorname{Hom}_{\left(\mathcal{H}_{P^{\prime}}^{+}, j_{P^{\prime}}^{+}\right)}\left(\mathcal{H}_{P^{\prime}}, \pi\right)=0$ where $P^{\prime}=$ $n_{w_{G} w_{P}} P^{\mathrm{op}} n_{w_{G} w_{P}}^{-1}$. Let $\lambda=\lambda_{P^{\prime}}^{+} \in Z\left(W_{P^{\prime}}(1)\right)$ be as in Proposition 2.5, Then again $\lambda$ satisfies the condition of the above lemma. Take $n \in \mathbb{Z}_{>0}$ such that $\pi\left(z_{\mathcal{O}}^{n}\right)=0$. Let $\varphi \in \operatorname{Hom}_{\left(\mathcal{H}_{P^{\prime}}^{+}, j_{P^{\prime}}^{+}\right)}\left(\mathcal{H}_{P^{\prime}}, \pi\right)$ and $X \in \mathcal{H}_{P^{\prime}}$. Since $j_{P^{\prime}}^{+}\left(E_{o_{-}, P^{\prime}}^{P^{\prime}}(\lambda)\right)=E_{o_{-}}(\lambda)$ by Lemma 2.6, with the previous lemma, we have

$$
\begin{aligned}
\varphi(X) & =\varphi\left(X E_{o_{-, P^{\prime}}}^{P^{\prime}}(\lambda)^{-(n+1)}\right) j_{P^{\prime}}^{+}\left(E_{o_{-, P^{\prime}}}^{P^{\prime}}(\lambda)^{n+1}\right) \\
& =\varphi\left(X E_{o_{-, P^{\prime}}}^{P^{\prime}}(\lambda)^{-(n+1)}\right) E_{o_{-}}(\lambda)^{n+1} \\
& =\varphi\left(X E_{o_{-, P^{\prime}}}^{P^{\prime}}(\lambda)^{-(n+1)}\right) z_{\mathcal{O}}^{n} E_{o_{-}}(\lambda)=0 .
\end{aligned}
$$

We get the proposition. 
5.7. Simple modules. Assume that $C$ is an algebraically closed field of characteristic $p$.

Theorem 5.20. Let $P$ be a parabolic subgroup, $\sigma$ a simple supersingular right $\mathcal{H}_{P}$-module and $Q$ a parabolic subgroup between $P$ and $P(\sigma)$. For a parabolic subgroup $R$, we have

$$
L_{R}(I(P, \sigma, Q))= \begin{cases}I_{R}(P, \sigma, Q \cap R) & \left(P \subset R, \Delta(\sigma) \subset \Delta_{Q} \cup \Delta_{R}\right), \\ 0 & (\text { otherwise }) .\end{cases}
$$

and

$$
R_{R}(I(P, \sigma, Q))= \begin{cases}I_{R}(P, \sigma, Q) & (Q \subset R), \\ 0 & (\text { otherwise }) .\end{cases}
$$

Proof. By Corollary [5.8, we have

$$
L_{R}(I(P, \sigma, Q))=L_{R}\left(I_{P(\sigma)}\left(\operatorname{St}_{Q}^{P(\sigma)}(\sigma)\right)\right)=I_{P(\sigma) \cap R}^{R}\left(L_{P(\sigma) \cap R}^{P(\sigma)}\left(\operatorname{St}_{Q}^{P(\sigma)}(\sigma)\right)\right)
$$

If $\Delta_{Q} \cup\left(\Delta(\sigma) \cap \Delta_{R}\right) \neq \Delta(\sigma)$, then it is zero by Proposition 5.10. Since $Q \subset$ $P(\sigma)$, we have $\Delta_{Q} \subset \Delta(\sigma)$. Hence $\Delta_{Q}=\Delta(\sigma) \cap \Delta_{Q}$ and, therefore we have $\Delta_{Q} \cup\left(\Delta(\sigma) \cap \Delta_{R}\right)=\Delta(\sigma) \cap\left(\Delta_{Q} \cup \Delta_{R}\right)$. Hence $\Delta_{Q} \cup\left(\Delta(\sigma) \cap \Delta_{R}\right)=\Delta(\sigma)$ if and only if $\Delta(\sigma) \subset \Delta_{Q} \cup \Delta_{R}$. Also from Proposition [5.10, if $\Delta(\sigma) \subset \Delta_{Q} \cup \Delta_{R}$, we have

$$
L_{R}(I(P, \sigma, Q))=I_{P(\sigma) \cap R}^{R}\left(\operatorname{St}_{Q \cap R}^{P(\sigma) \cap R}\left(L_{P \cap R}^{P}(\sigma)\right)\right),
$$

here we use $Q \subset P(\sigma)$. By Proposition 5.18, this is zero if $P \cap R \neq P$, namely $P \not \subset R$. If $P \subset R$, then

$$
L_{R}(I(P, \sigma, Q))=I_{P(\sigma) \cap R}^{R}\left(\operatorname{St}_{Q \cap R}^{P(\sigma) \cap R}(\sigma)\right)=I(P, \sigma, Q \cap R) .
$$

For the functor $R$, by Proposition [5.1, we have

$$
R_{R}(I(P, \sigma, Q))=R_{R}\left(I_{P(\sigma)}\left(\operatorname{St}_{Q}^{P(\sigma)}(\sigma)\right)\right)=I_{P(\sigma) \cap R}^{R}\left(R_{P(\sigma) \cap R}^{P(\sigma)}\left(\operatorname{St}_{Q}^{P(\sigma)}(\sigma)\right)\right) .
$$

By Proposition 5.11, this is zero if $\Delta_{Q} \neq \Delta_{Q \cap R \cap P(\sigma)} \cup \Delta_{P}$. Note that we have $Q \cap R \cap P(\sigma)=Q \cap R$ since $Q \subset P(\sigma)$. If $\Delta_{Q}=\Delta_{Q \cap R} \cup \Delta_{P}$, then we have

$$
R_{R}(I(P, \sigma, Q))=I_{P(\sigma) \cap R}^{R}\left(\operatorname{St}_{Q \cap R}^{P(\sigma) \cap R}\left(R_{P \cap R}^{P}(\sigma)\right)\right) .
$$

By Proposition 5.18, this is zero if $P \cap R \neq P$. If $P \cap R=P$, namely $P \subset R$, then we have

$$
R_{R}(I(P, \sigma, Q))=I_{P(\sigma) \cap R}^{R}\left(\operatorname{St}_{Q \cap R}^{P(\sigma) \cap R}(\sigma)\right)=I_{R}(P, \sigma, Q \cap R) .
$$

Hence we get

$$
R_{R}(I(P, \sigma, Q))= \begin{cases}I_{R}(P, \sigma, Q \cap R) & \left(P \subset R, \Delta_{Q}=\Delta_{Q \cap R} \cup \Delta_{P}\right), \\ 0 & \text { (otherwise) }\end{cases}
$$

If $P \subset R$, then $\Delta_{Q \cap R} \supset \Delta_{P}$. Hence $\Delta_{Q}=\Delta_{Q \cap R} \cup \Delta_{P}$ implies $\Delta_{Q}=\Delta_{Q \cap R}$, namely $Q \subset R$. On the other hand, if $Q \subset R$ then $P \subset R$ and $\Delta_{Q}=$ $\Delta_{Q} \cup \Delta_{P}=\Delta_{Q \cap R} \cup \Delta_{P}$. We get the theorem. 


\section{REFERENCES}

[Abe] N. Abe, Modulo p parabolic induction of pro-p-Iwahori Hecke algebra, J. Reine Angew. Math., DOI:10.1515/crelle-2016-0043.

[AHHV] N. Abe, G. Henniart, F. Herzig, and M.-F. Vigneras, A classification of irreducible admissible mod $p$ representations of $p$-adic reductive groups, Journal of the AMS, DOI:10.1090/jams/862.

[AHVa] N. Abe, G. Henniart, and M.-F. Vignéras, Modulo p representations of reductive p-adic groups: functorial properties, in preparation.

[AHVb] N. Abe, F. Herzig, and M.-F. Vignéras, Change of weight and inverse Satake isomorphism, in preparation.

[Deo77] V. V. Deodhar, Some characterizations of Bruhat ordering on a Coxeter group and determination of the relative Möbius function, Invent. Math. 39 (1977), no. 2, 187-198.

[Oll10] R. Ollivier, Parabolic induction and Hecke modules in characteristic $p$ for $p$-adic $\mathrm{GL}_{n}$, Algebra Number Theory 4 (2010), no. 6, 701-742.

[Oll14] R. Ollivier, Compatibility between Satake and Bernstein isomorphisms in characteristic p, Algebra Number Theory 8 (2014), no. 5, 1071-1111.

[Oll15] R. Ollivier, An inverse Satake isomorphism in characteristic $p$, Selecta Math. (N.S.) 21 (2015), no. 3, 727-761.

[Vig14] M.-F. Vignéras, The pro-p-Iwahori-Hecke algebra of a reductive p-adic group, II, Münster J. Math. 7 (2014), no. 1, 363-379.

[Vig15a] M.-F. Vignéras, The pro-p-Iwahori Hecke algebra of a p-adic group III, J. Inst. Math. Jussieu (2015), 1-38.

[Vig15b] M.-F. Vignéras, The pro-p Iwahori Hecke algebra of a reductive p-adic group, $V$ (parabolic induction), Pacific J. Math. 279 (2015), no. 1-2, 499-529.

[Vig16] M.-F. Vigneras, The pro-p-Iwahori Hecke algebra of a reductive p-adic group I, Compos. Math. 152 (2016), no. 4, 693-753.

Department of Mathematics, Hokkaido University, Kita 10, Nishi 8, KitaKu, SAPporo, HoKKaido, 060-0810, Japan

E-mail address: abenori@math.sci.hokudai.ac.jp 